\title{
SAFETY AND OPERATIONS GUIDANCE FOR USING TIME-OF- DAY PROTECTED-PERMISSIVE LEFT-TURN PHASING USING FLASHING YELLOW ARROWS
}

\author{
A Thesis \\ Presented to \\ The faculty of the School of Engineering and Applied Sciences \\ University of Virginia
}

In Partial Fulfillment

of the requirements for the Degree

Master of Science (Civil Engineering)

F. Adam King

December 2017 


\author{
APPROVAL SHEET \\ This thesis is submitted in partial fulfillment of the \\ requirements for the degree of \\ Masters of Science (Civil Engineering)
}

F. Adam King

This thesis has been read and approved by the Examining Committee:

Dr. Michael D. Fontaine, Thesis Advisor

Dr. B. Brian Park

Dr. T. Donna Chen

Accepted for the School of Engineering and Applied Sciences:

Dean, School of Engineering and Applied Sciences December 2017 


\section{ACKNOWLEDGEMENTS}

First and foremost, I would like to express my gratitude towards my advisor, Dr. Michael D. Fontaine for allowing me the opportunity to pursue my Master's Degree and learn from him. I cannot imagine a better advisor than Dr. Fontaine, as his enthusiasm was truly infectious, and he was always passionate about the research we conducted. He also made sure that I was on track throughout my research and was always available for me when I had questions.

I would also like to acknowledge my committee members, Dr. B. Brian Park and Dr. T. Donna Chen for their support, as well as all of the professors that I have learned from.

I am also very grateful for the help and support of Dr. Justice Appiah and Mr. Benjamin H. Cottrell. Through countless research meetings and questions, they made my research

possible. I specifically would like to thank Dr. Appiah for his assistance throughout the project and his scripting expertise, as he saved me an immeasurable number of hours automating the simulations.

This project also would not have been possible if not for my high school mentor, Aaron Small, as I would not have pursued a degree in Civil Engineering without his enthusiasm and guidance with his mentoring. Also, I would like to thank everyone who has supported me within VDOT through my experience in the Internship and Scholarship programs; with their knowledge and support I gained valuable experience and knowledge of transportation and traffic engineering.

I am also extremely glad that I had the opportunity to study here at the University of Virginia. In the year and a half that I have been here, I have learned a great deal from my professors and peers, and have had a great time getting to know many people in the Department.

Finally, I would like to express my deep gratitude towards my family, friends, and 
girlfriend for their continued support throughout the pursuit of my education. They have been with me every step of the way, and have gotten me through many challenges through their love and care. 


\section{ABSTRACT}

Selection of the left-turn phasing mode is a crucial decision for the safe and efficient movement of left-turning traffic at signalized intersections. Because of different safety and operational effects caused by the signal mode, the two must be evaluated concurrently and be balanced based on capacity and crash potential when comparing protected-only, permissive-only, and protected-permissive (PPLT) modes. This left-turn phasing mode choice can be made on a time-of-day basis, so that changing traffic conditions are accommodated appropriately. This thesis seeks to define guidance that field traffic engineers can use to select the appropriate leftturn mode based on prevailing traffic conditions by time-of-day. In particular, guidance on the use of PPLT with flashing yellow arrows was of interest to the Virginia Department of Transportation (VDOT).

Prior to developing time-of-day guidance, the overall safety effects of converting left-turn phase modes and indications needed to be explored. This thesis examined the impact of converting from a circular green "ball" display for the permissive portion of PPLT phasing to the flashing yellow arrow (FYA) signal indication, as well as protected-only phasing to PPLT with FYA. To quantify these conversions, a before and after evaluation of signal conversions was performed using the Empirical Bayes (EB) approach to develop crash modification factors (CMFs) from data for 37 intersections in Virginia. Statistically significant CMFs were found for the PPLT with green ball to PPLT-FYA conversion for all severities and angle crash types as $0.71,0.62$, and 0.77 for all, 3-, and 4-leg intersections. For conversions from protected-only to PPLT-FYA, CMFs of 1.47 and 2.56 for all and angle crash types for all severities, as well as CMFs of 1.57 and 3.30 for all and angle types for fatal and injury severities were determined.

In evaluating different left-turn phasing modes on a time-of-day basis, crash risk, left-turn 
conflict, and capacity prediction models for permissive-only and PPLT modes were developed using simulation data. A total of 750 unique scenarios based on different combinations of intersection characteristics, traffic signal parameters, and traffic volumes were simulated in VISSIM and trajectory files were processed in SSAM for the number of conflicts per scenario. From the outputs from the simulation models, prediction models for determining left-turn capacities and the number of left-turn conflicts per 100 left-turning vehicles were created using multiple regression. Additionally, a crash risk model was created. A final model predicting the average crash risk per hour based on the predicted number of conflicts was developed to be used as a crash risk assessment tool. The three models created were incorporated into a new tool for the evaluation of signal phasing based on time-of-day. This spreadsheet incorporated all of the resulting models into one tool, and should be used by VDOT engineers in determining phasing mode on a time-of-day basis. 
TABLE OF CONTENTS

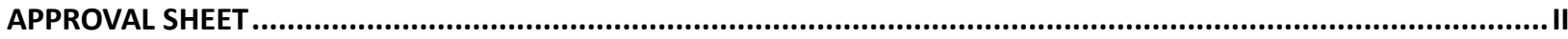

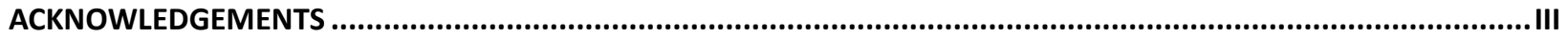

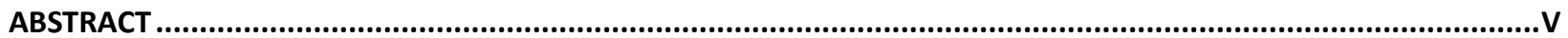

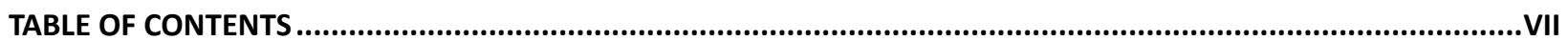

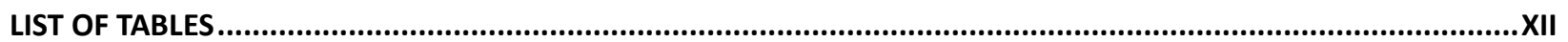

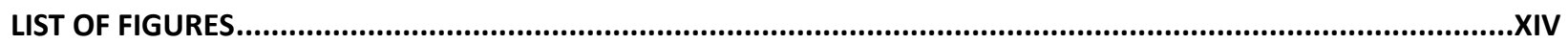

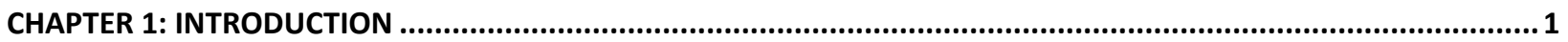

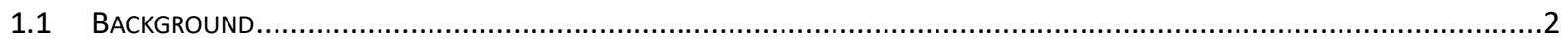

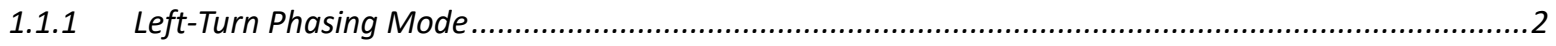

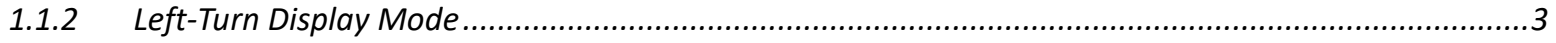

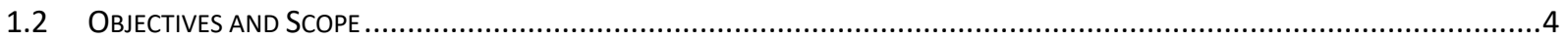

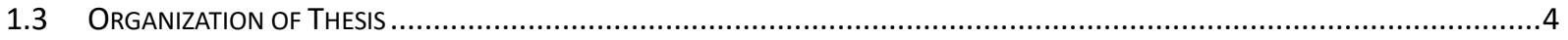

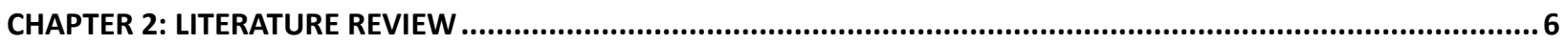

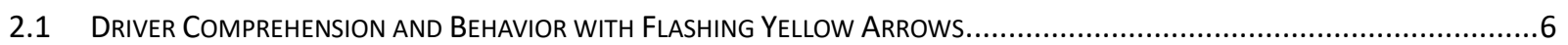

2.1.1 Driver Comprehension of Left-Turn Signal Displays ................................................................6

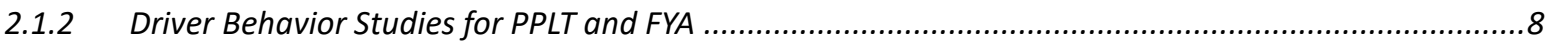

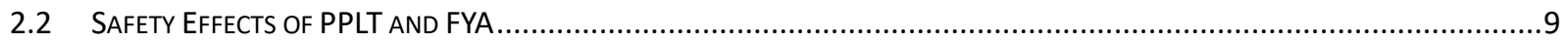

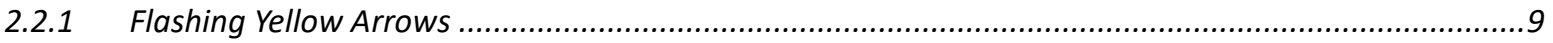

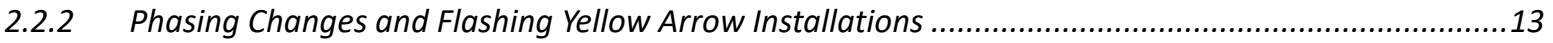

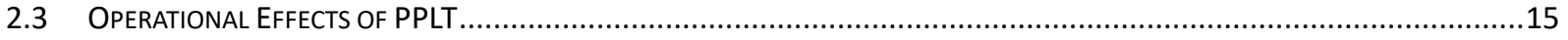

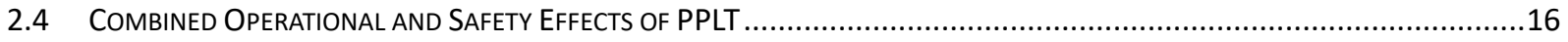

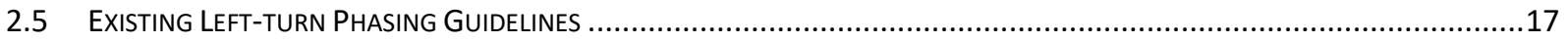




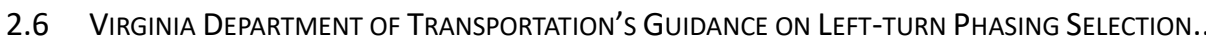

2.7 SUMMARY AND GAPS IN EXISTING LITERATURE.

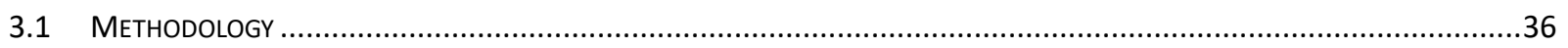

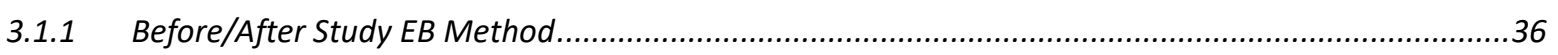

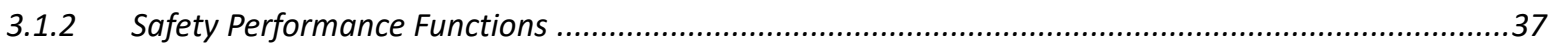

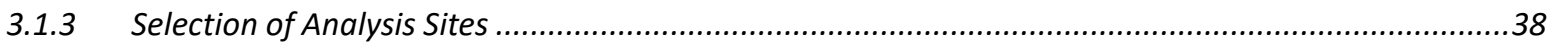

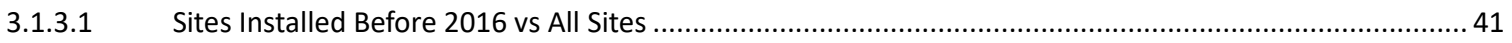

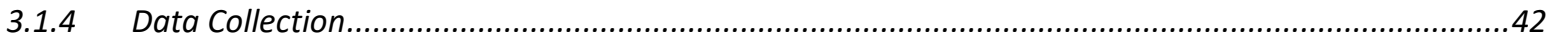

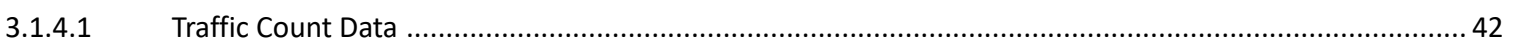

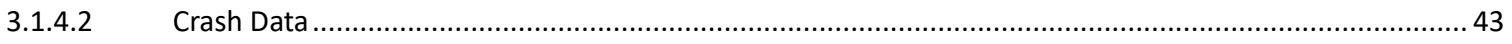

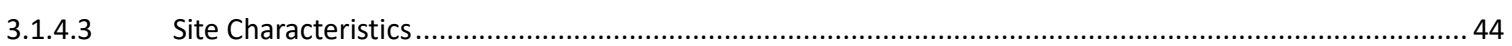

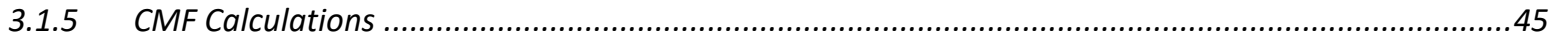

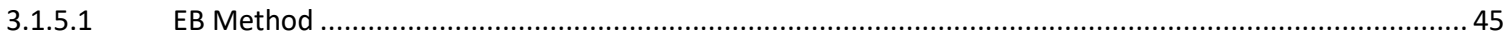

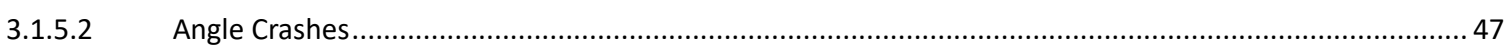

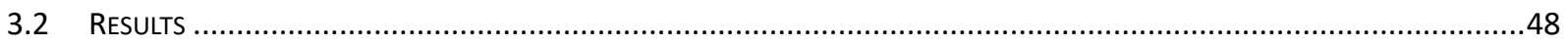

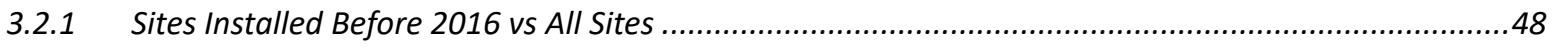

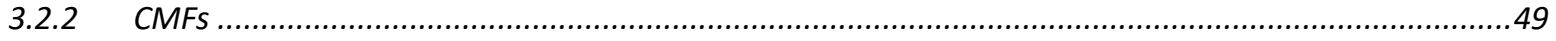

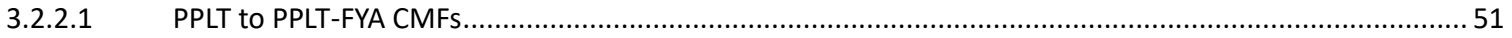

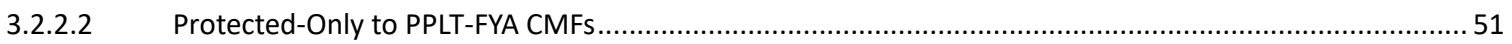

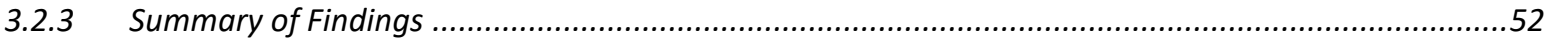

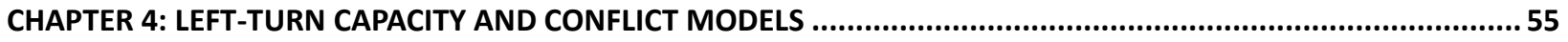

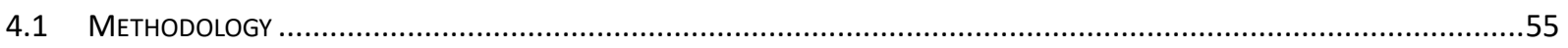

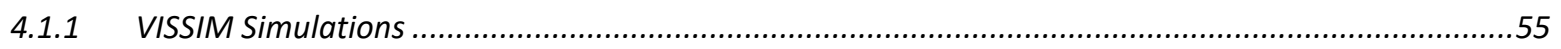

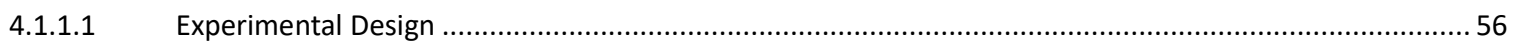

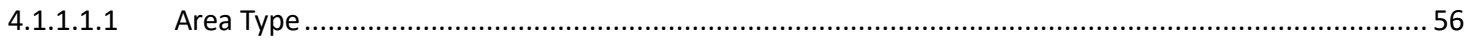




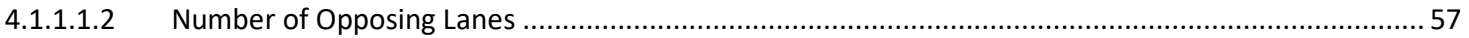

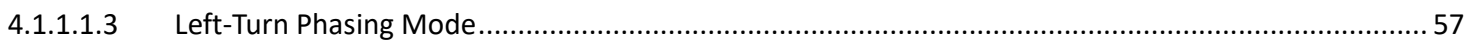

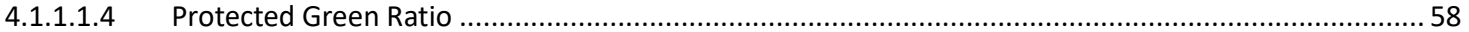

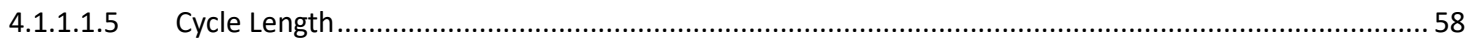

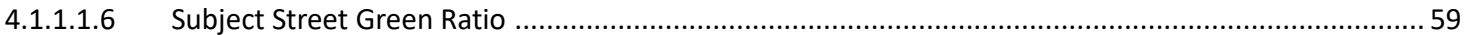

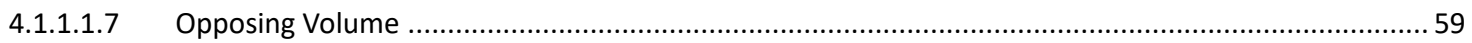

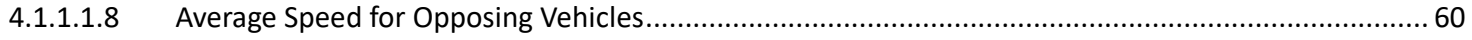

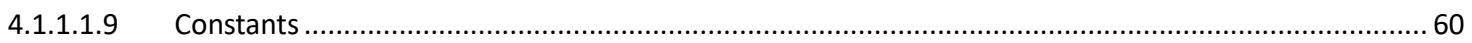

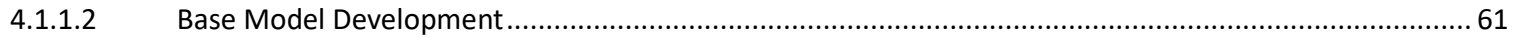

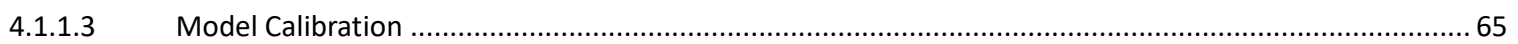

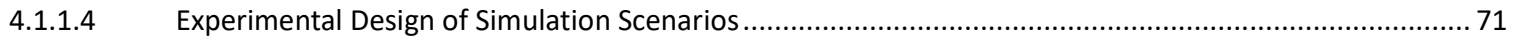

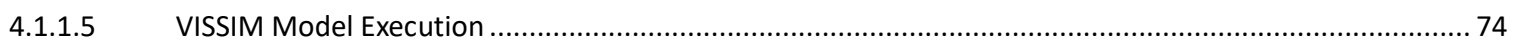

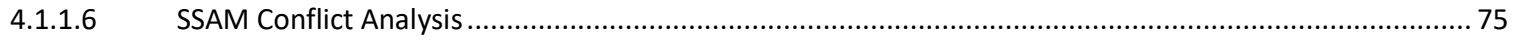

4.1.2 Capacity and Conflict Prediction Model Development...............................................................77

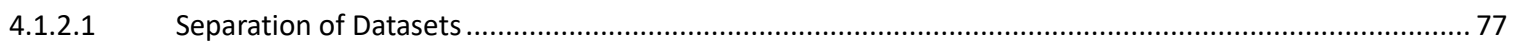

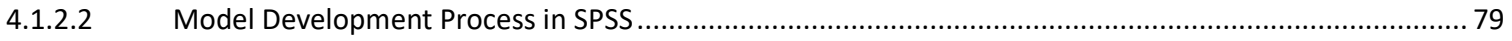

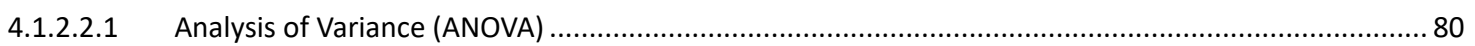

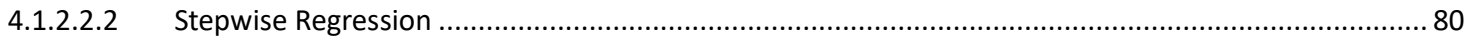

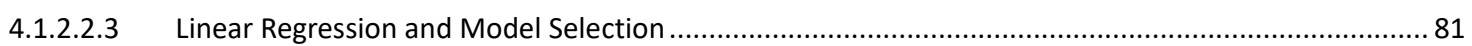

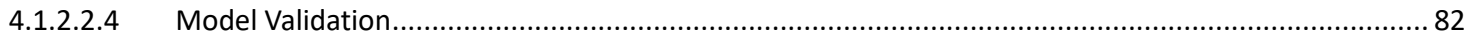

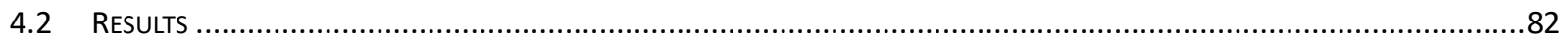

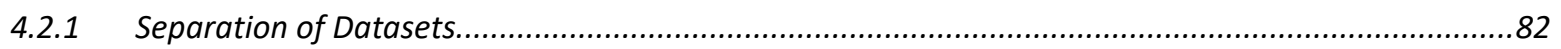

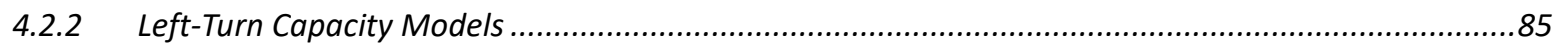

4.2.2.1 PPLT Capacity Model for Non-Zero Permissive Capacities ............................................................ 86

4.2.2.2 PPLT Capacity Model for Zero-Permissive Capacities ….................................................................... 89

4.2.2.3 Permissive-Only Capacity Model for Non-Zero Permissive Capacities ...............................................91

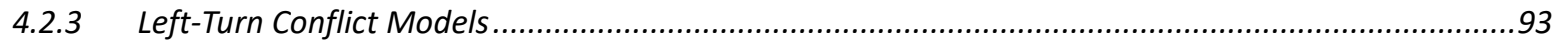

4.2.3.1 PPLT Left-Turn Conflict Model for Non-Zero Permissive Capacities ..................................................93 
4.2.3.2 Permissive-Only Left-Turn Conflict Model for Non-Zero Permissive Capacities ....................................96

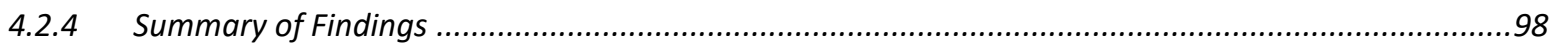

CHAPTER 5: RISK ASSESSMENT MODEL FOR TIME-OF-DAY SAFETY ANALYSIS............................................... 100

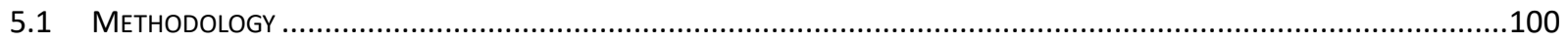

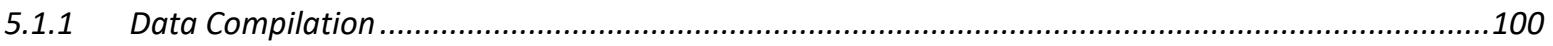

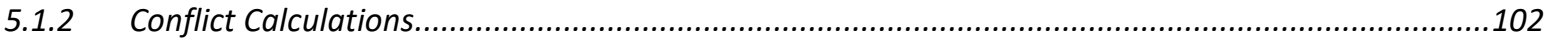

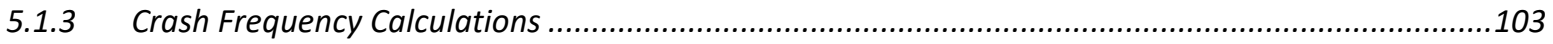

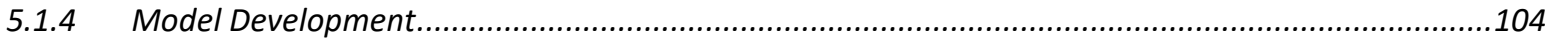

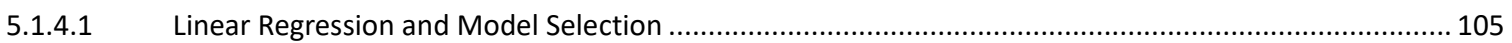

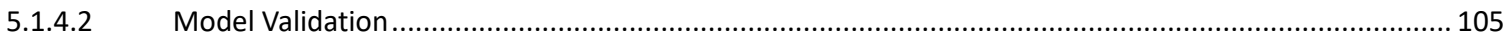

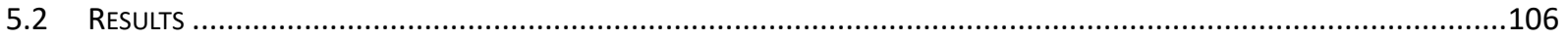

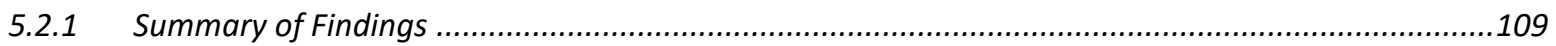

CHAPTER 6: SPREADSHEET TOOL FOR PRACTITIONERS ..................................................................... 111

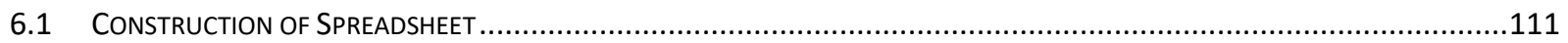

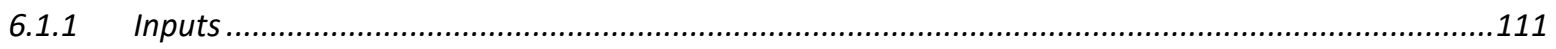

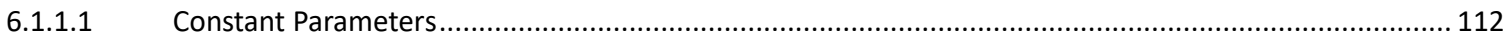

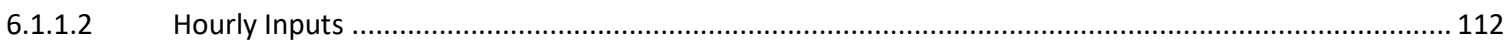

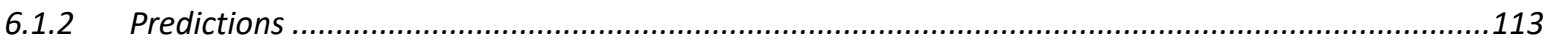

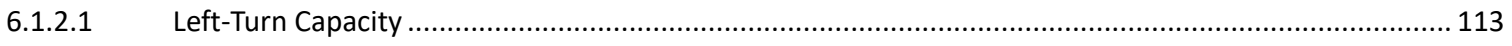

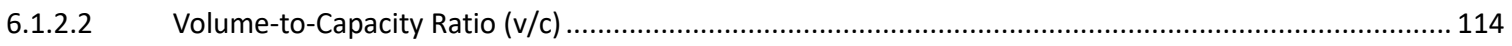

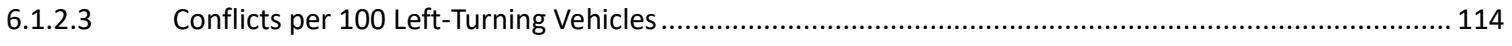

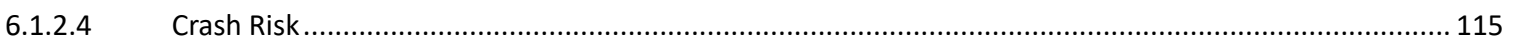

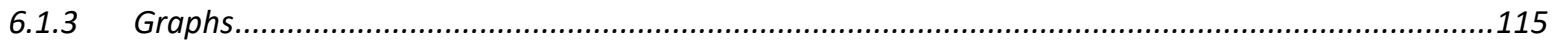

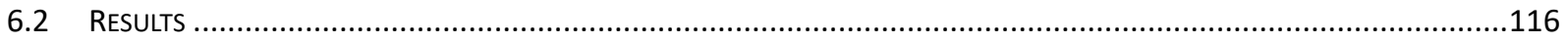

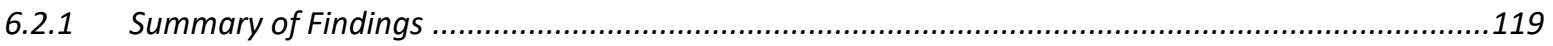




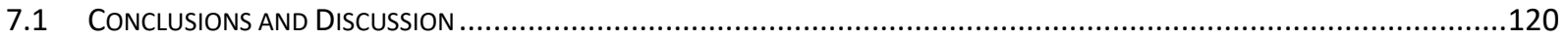

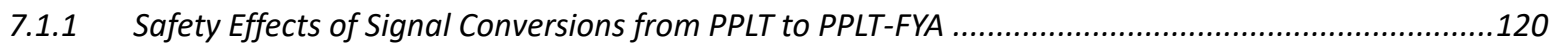

7.1.2 Safety Effect of Signal Conversions from Protected-Only to PPLT-FYA ......................................120

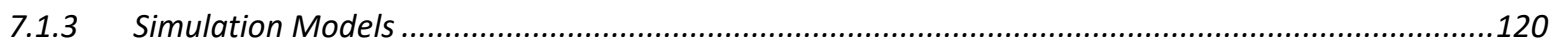

7.1.4 Decision Support Tool........................................................................................................ 121

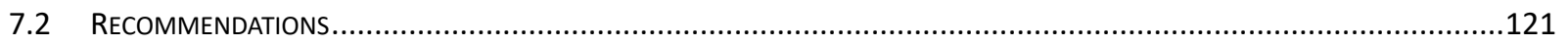

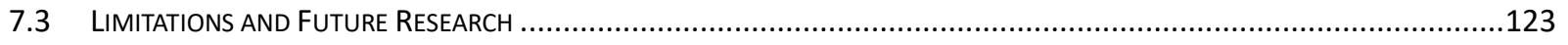

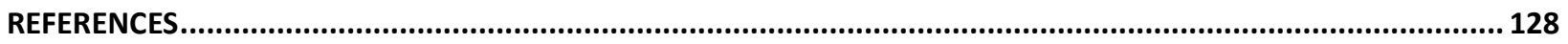

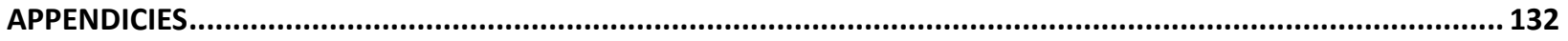

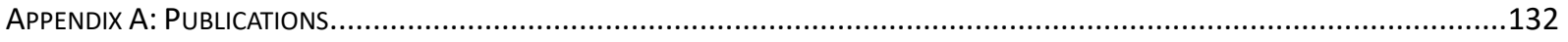

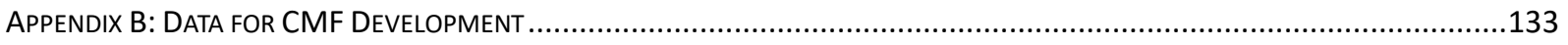

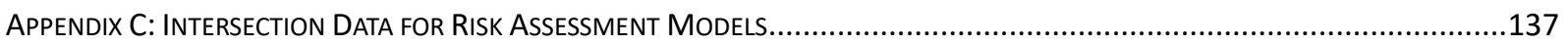




\section{LIST OF TABLES}

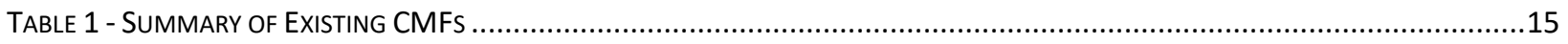

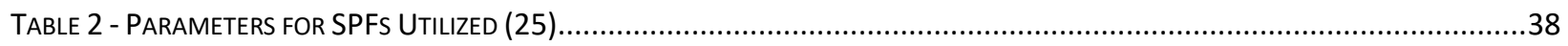

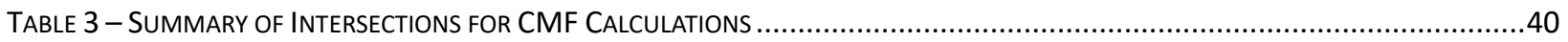

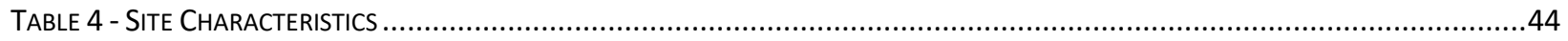

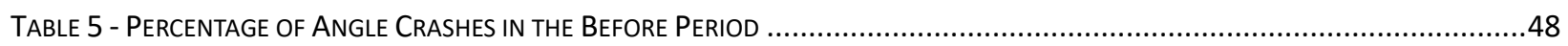

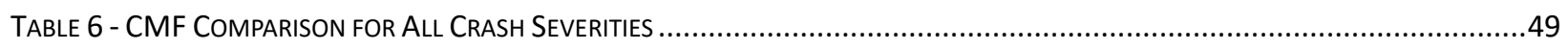

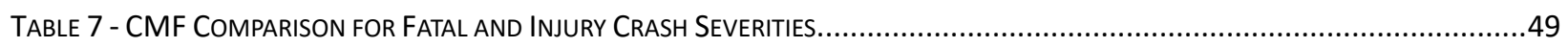

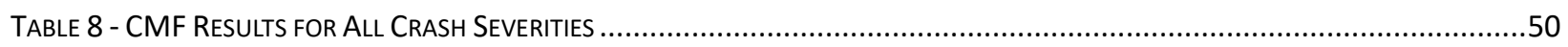

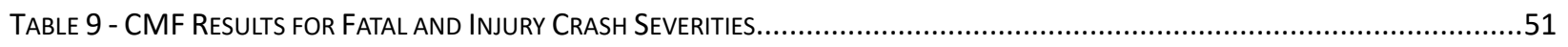

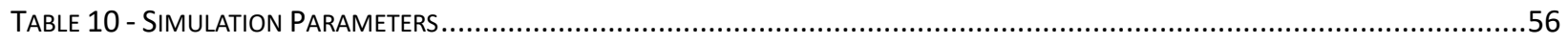

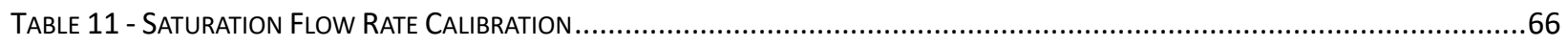

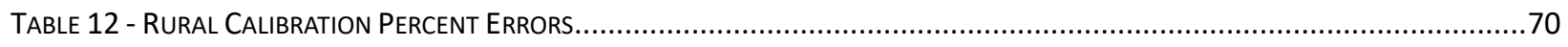

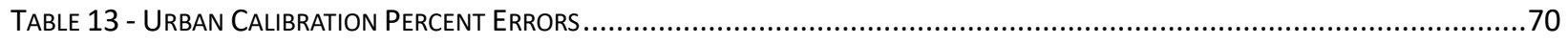

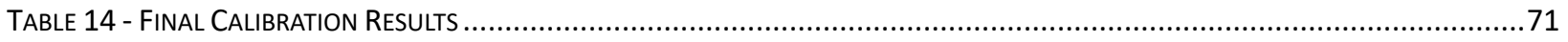

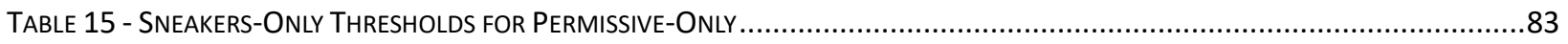

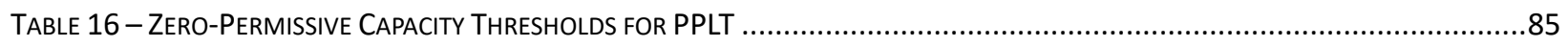

Table 17 - ANOVA Results for PPLt Capacity Model for Non-Zero Permissive CaPaCities.............................................87

Table 18 - Regression Parameters for PPlt Capacity Model for Non-Zero Permissive Capacities ...................................89

TAble 19 - Validation Results for PPlt CaPACIty Models for Non-Zero Permissive CAPACITIES .........................................89

TABLE 20 - ANOVA ReSUltS For PPLT CAPACITY Model FOR Zero-PERMISSIVE CAPACITIES ...............................................90

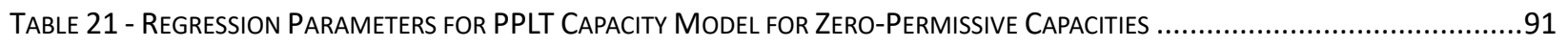

TABLE 22 - Validation ReSUltS FOR PPLT CAPACITY ModelS For ZeRo-PermisSIVE CAPACITIES ...............................................91

Table 23 - ANOVA Results for Permissive-Only Capacity Model for Non-Zero Permissive Capacities...............................92

Table 24 - Regression Parameters for Permissive-Only Capacity Model for Non-Zero Permissive Capacities .....................93 
Table 25 - Validation Results for Permissive-Only Capacity Models for Non-Zero Permissive Capacities.

TABle 26 - ANOVA Results for PPLt Conflict Model for Non-Zero Permissive Capacities

Table 27 - Regression Parameters for PPLt Conflict Model for Non-Zero Permissive Capacities...................................95

Table 28 - Validation Results for PPLt Conflict Models for Non-Zero Permissive Capacities .......................................95

Table 29 - ANOVA Results for Permissive-Only Conflict Model for Non-Zero Permissive Capacities ..............................96

Table 30 - Regression Parameters for Permissive-Only Conflict Model for Non-Zero Permissive Capacities.....................97

Table 31 - Validation Results for Permissive-Only Conflict Models for Non-Zero Permissive Capacities ..........................97

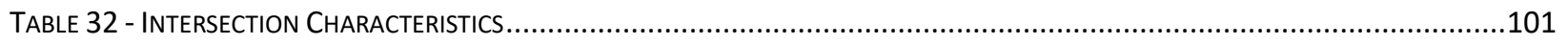

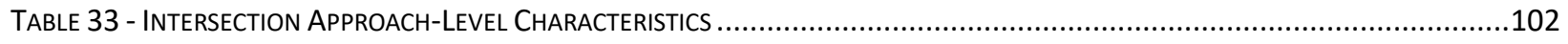

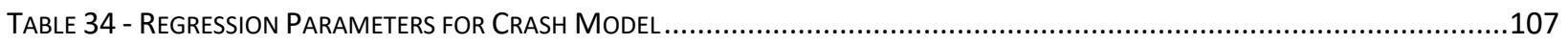

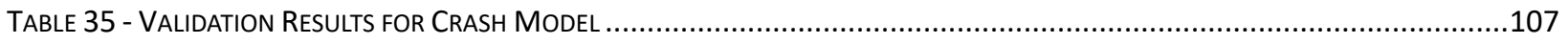

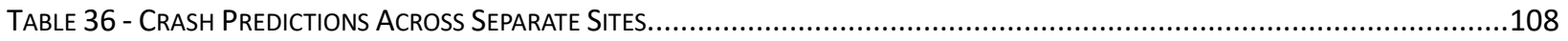

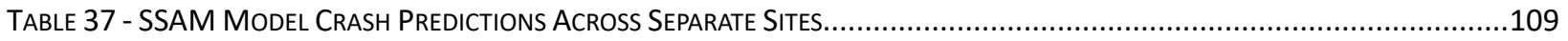

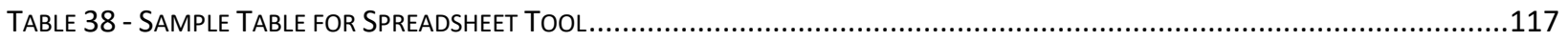




\section{LIST OF FIGURES}

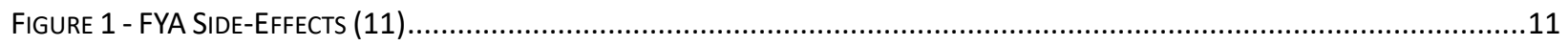

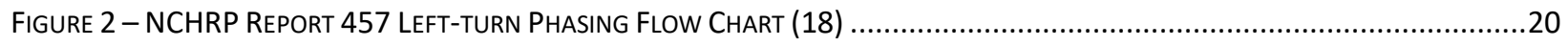

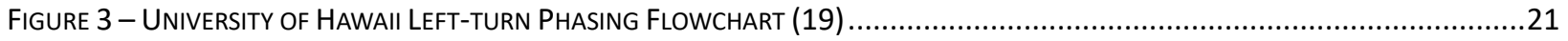

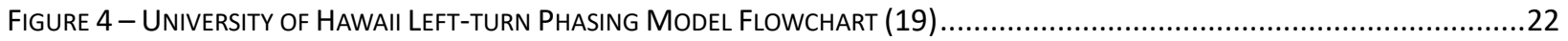

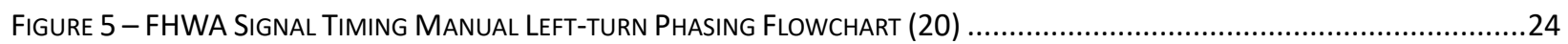

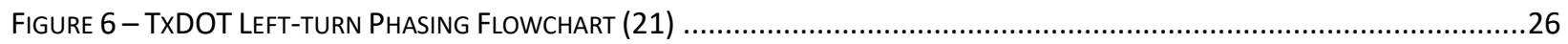

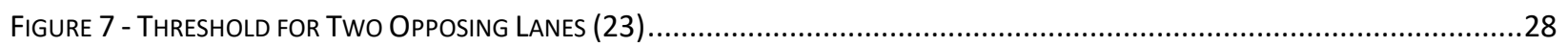

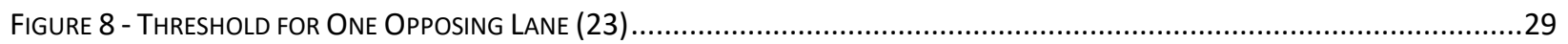

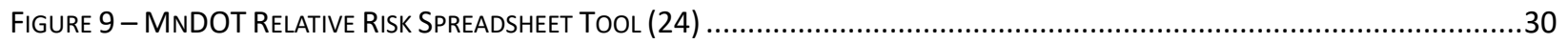

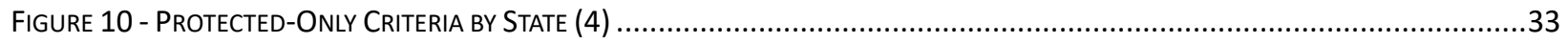

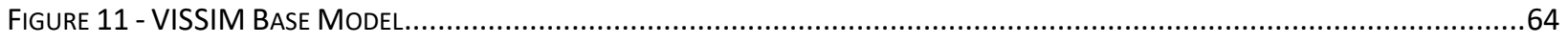

Figure 12 - QueUe AcCumulation Polygon for ProteCted Movements (27) ...........................................................68

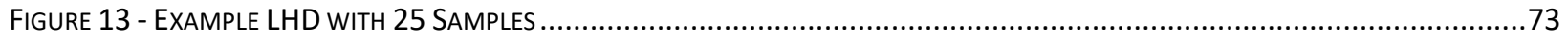

FIGURE 14 - EXAMPLE FULL-FACTORIAL DESIGN WITH 25 SAMPLES .................................................................................73

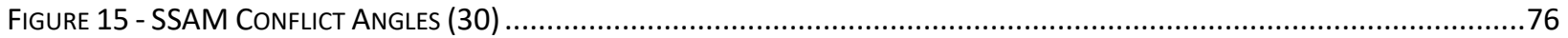

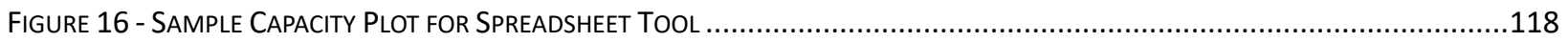

FigURE 17 - SAMPLE CRASH FreQUenCy PLot for SPREAdSHEet ToOL .....................................................................118 


\section{CHAPTER 1: INTRODUCTION}

Left-turn control mode selection at signalized intersections is a significant decision that an engineer must make in signal design. If they select a phasing control mode that is too restrictive, safety may improve at the cost of decreased capacity, and if they select a mode that is less restrictive, capacity may improve at the cost of safety. Also, if only operations are considered when making the selection, unintended safety consequences may be realized, and vice-versa. Because of this, the engineer must carefully balance operational efficiency and driver safety when determining an appropriate phasing mode for left-turns on a signal approach. Additionally, they should ensure the efficient operation of the intersection as a whole is not compromised by the left-turn phase choice.

While left-turn mode is often the same throughout the entire day, there is increasing interest in Virginia in deploying left-turn modes that can vary by time-of-day. As traffic volumes vary throughout the course of the day, left-turn mode could vary so that the optimal timing and control are given at all times. Most existing left-turn phase selection guidelines focus on selecting a single mode for the entire day, so the Virginia Department of Transportation (VDOT) has become interested in developing time-of-day based signal phasing guidance. In particular, VDOT is interested in applications of the flashing yellow arrow (FYA) display for permissive or protected-permissive left turn (PPLT) phasing, as the FYA has been proven to reduce the number of left-turn crashes when converted from a green ball indication (1) (2). Since its incorporation in the 2009 Manual on Uniform Traffic Control Devices (MUTCD) (3), VDOT has been retrofitting intersections with the FYA display based on the results shown in these past studies.

The research presented in this document quantifies the safety implications of changes in signal display and control mode in terms of Crash Modification Factors (CMFs), identifies 
factors that affect left-turn capacity and conflicts for PPLT and permissive left-turn modes through microscopic traffic simulations, creates prediction models for left-turn capacity and conflicts for PPLT and permissive left-turn modes, and relates left-turn conflicts to left-turn crash risk. This research was used to develop a spreadsheet tool to aid VDOT engineers in the decision process for left-turn control mode determination on a time-of-day basis.

\subsection{BACKGROUND}

\subsubsection{Left-Turn Phasing Mode}

As defined in the Virginia Department of Transportation's (VDOT's) Guidance for Determination and Documentation of Left-Turn Phasing Mode (4), three different signal control modes are used in Virginia: protected-only, permissive-only, and protected-permissive (PPLT). With protected-only left-turn phasing, left-turning drivers are allowed to execute their turning movement only when they are given complete right-of-way over other opposing movements. This significantly improves safety by separating conflicting flows, but may negatively impact operations on other approaches. For the permissive-only left-turn mode, the left-turn movement is never given full right of way, but rather allows left-turning vehicles to turn while yielding to opposing traffic. This mode increases the risk of left-turn related crashes, but minimizes operational impacts to opposing movements. Finally, protected-permissive phasing uses a combination of the two aforementioned modes to provide right-of-way to left-turning vehicles for a portion of the signal cycle, and permitted left-turns during the opposing through phase.

Since many different factors affect traffic signal operations and safety, there is not a single left-turn mode that works for every intersection. The optimal control mode will change based on parameters affecting the intersection, thus the left-turn mode decision must be made on 
a case-by-case basis. When an engineer decides which control mode to utilize for the left-turns at each of the approaches at an intersection, they must consider several variables concurrently, such as signal timing, traffic volumes, and crash history, to name a few. The decision is often complex, and in Virginia it is not a standardized process across the state. Additionally, there are currently no methods that integrate capacity and safety considerations in this decision. Without the ability for engineers to evaluate these concurrently, they are unable to determine the most effective control mode for each hour of the day.

\subsubsection{Left-Turn Display Mode}

If a driver misunderstands a signal indication and makes an incorrect action, a crash may occur. The green arrow display for protected left-turns is typically well understood, but there can be some confusion associated with different permissive portion displays. Traditionally, the circular green "ball" indication has been used to inform the driver that they may turn left while yielding to the opposing direction. In recent years, the flashing yellow arrow (FYA) display has started to replace the traditional green ball indication in signaling a permissive left-turns, as this display has been proven to be better understood by drivers (5) (6) (7) (8) (9).

Although the safety effects of conversion from the green ball to the FYA have been quantified (1) (2), the effects of this conversion for particular intersection geometries have not been examined, and the effects of this conversion within PPLT configurations is not as well understood as with permitted phasing. Because of this, accurate estimates as to how the conversion will affect a particular intersection are not available, thus separate 3- and 4-leg intersection crash modification factors (CMFs) should be developed. Additionally, CMFs for the conversions' effect on angle crashes specifically have not been quantified. Since the crash type 
field on crash reports in Virginia typically only include angle crash types (as opposed to a leftturn specific crash type), an angle crash CMF would be more useful to engineers in Virginia, as it would better match the data available to them. Angle crashes are also considered to be the target crash type affected by left-turn displays, as left-turn crashes occur between turning vehicles and opposing through vehicles, on an angle.

\subsection{OBJECTIVES AND SCOPE}

The purpose of this study is to provide VDOT engineers with a better understanding of the impacts of protected-permissive phasing using the FYA display on safety and operational efficiency of signalized intersections. The specific objectives of this research are to:

1. Quantify the safety effects of converting the PPLT display from green ball to FYA. Impacts on crash type and severity are evaluated, as well as the different effects of intersection geometries.

2. Quantify the safety effects of converting left-turn phasing from protected-only phasing to PPLT with FYA.

3. Develop a left-turn phasing assessment tool for VDOT engineers to weigh the operational and safety trade-offs of protected-only, permissive-only, and PPLT modes, based on time-of-day.

The first two objectives were assessed using field data from VDOT FYA installations. The methodology for the third was developed using simulated data.

\subsection{ORGANIZATION OF THESIS}

This document is separated into six chapters. Following this chapter, a summary of 
existing literature relating to the research presented in this document is provided, along with an explanation of the gaps in past research. Chapters 3, 4, and 5 explain the procedures and results from the development of the CMFs, simulation models, and risk assessment models, respectively. Chapter 6 provides an explanation of the development of a guidance tool for engineers, and finally, Chapter 7 draws conclusions from the results, discusses the implications of the conclusions, and describes possible future work. 


\section{CHAPTER 2: LITERATURE REVIEW}

As a part of this research, a review of existing literature and studies was conducted to identify the state of the art and the state of the practice for flashing yellow arrow (FYA) applications for protected-permissive left-turn (PPLT) phasing. Papers, journal articles, and agency guidance documents were analyzed to better understand the effects of PPLT and FYA on intersection operations and safety, as well as to understand how other state agencies developed their guidelines on the use of PPLT and FYA.

\subsection{DRIVER COMPREHENSION AND BEHAVIOR WITH FLASHING YELLOW ARROWS}

\subsubsection{Driver Comprehension of Left-Turn Signal Displays}

The University of Massachusetts published a report to evaluate different signal displays, including FYA in 2001 (5). This study evaluated the effect of different permitted signal indications (green ball, flashing red arrow, flashing red ball, flashing yellow arrow, and flashing

yellow ball), as well as other factors such as geographical location, driver demographics, and signal head arrangement, on a driver's correct understanding of their right-of-way. In gathering data for the research, the team presented static photographs of different intersection and signal characteristics to 2,465 drivers with 30 scenarios per driver. The study found that there was a higher level of comprehension and a lower fail critical rate with flashing permitted signal indications, and that all flashing red and yellow ball and arrow indications had significantly higher correct response rates than green ball indications. Additionally, the team indicated that further research into flashing permitted indications should be conducted through driver simulations and field studies. 
In 2003, NCHRP Report 493 reviewed existing literature on signal displays for PPLT phasing and evaluated driver understanding of displays, crash data, and operational data to determine the effects of different displays on left-turn phasing under PPLT (6). The major findings from this study were that the FYA display was widely understood by drivers, a higher fail-safe response rate can be realized over a green ball display, and that the FYA display eliminates the "yellow trap." Thus, the researchers suggested that the FYA permissive indication be included in the next release of the Federal Highway Administration's (FHWA) MUTCD.

Following the 2001 University of Massachusetts study, another study was conducted to evaluate driver understanding of different signal displays using a driver simulator (7). In this study, two full-scale, fully-interactive driver simulators at the University of Massachusetts and at the Texas A\&M Transportation Institute were used to evaluate driver behavior in different scenarios of green ball and FYA signal indications. Additionally, a similar method to the static photographs used in the 2001 study was employed, but this time using a video-based static evaluation that simulated the signal display to create a more realistic scenario. For the driver simulator, it was found that the percentage of correct responses varied from $90 \%$ to $92 \%$, with no statistically significant differences between the different variables (indication, arrangement, through indication, and location). Also, average fail-critical response rates for the simulator were found to not be statistically different between signal indications. For the static evaluation, a higher correct response rate was observed for FYA rather than green ball. The study also found that generally the correct response rate for the simulator study was higher than that of the static evaluation, indicating that drivers use contextual information to make their turning decisions, rather than just the signal display indication. As a result of the study, it was determined that the FYA left-turn display had a lower fail-critical response rate than green ball and that it had a high 
level of driver comprehension.

Because of the findings of the above three studies, the FYA display was incorporated in to the 2009 MUTCD as an acceptable display for permissive portions of left-turn modes (3). With this inclusion, more states have started to convert signals from the traditional green ball display to FYA.

\subsubsection{Driver Behavior Studies for PPLT and FYA}

In 2013 a study evaluated driver behavior by studying real-world driver reactions to different permissive interval signal indications (8). To do this, the researchers collected video data and extracted information such as gap size accepted by left-turning vehicles, occurrences of adequate gap size being rejected, and comments on the actions (such as if the driver slowed down before approaching the turn). With the data, driver behavior was compared between green ball, flashing yellow arrow, and flashing red arrow signal display locations. Statistical comparisons of means and variances of the proportions of drivers' actions for the three display modes were completed. These comparisons yielded the percentages of drivers who completed safe actions, efficient actions, and the combination of safe and efficient actions for each signal display. These values were compared to determine if they were statistically significantly different from one another at the $95 \%$ level. The result of the analysis was that drivers made the safest and most efficient actions at intersections with FYA installed. Additionally, the study found that there was no significant difference in driver behavior between geographic areas that used multiple permissive indications and areas that used only one permissive indication.

In a joint research effort between the Indiana Department of Transportation and Purdue University, FYA installations were evaluated and recommendations were made for the 
widespread implementation of FYA in Indiana (9). The study used observational analyses, crash data, and survey data to create the recommendations for FYA. For the driver performance (observational) study, radar was used to collect acceleration and deceleration data to be compared at two intersections with PPLT-FYA and two with PPLT. In evaluating the data, average deceleration and acceleration were determined to be the same, thus there was no significant difference in driver performance between PPLT-FYA and PPLT. For the crash study, average crash rates were compared between the different display types, but there was no conclusive evidence that one was safer than the other. The final technique the researchers used, the driver survey, collected driver responses to theoretical situations with different signal head indications and orientations. From the surveys, it was determined that a majority of drivers gave correct responses, with few fail-critical responses. As a result of the research, the Department recommended that FYA be used as an alternative to green ball indications and that proper public education be provided.

\subsection{SAFETY EFFECTS OF PPLT AND FYA}

\subsubsection{Flashing Yellow Arrows}

Researchers at the University of North Carolina at Charlotte, in an attempt to illustrate safety implications of FYA installations, used the Empirical Bayes (EB) technique to analyze crashes at six intersections converted to FYA in Charlotte, North Carolina (10). These intersections used the green ball indication and were converted to FYA, though the report did not

specify if permissive-only or PPLT phasing was used at the study sites. In the study, the researchers developed Safety Performance Functions (SPFs) that predicted the number of crashes based on Average Annual Daily Traffic (AADT) and skewness of the intersection. From the 
analysis, the team found that five of the six intersections studied had odds ratios (ORs) of less than 1.0. With a small dataset, the researchers did not conclude that the FYA installations were effective in reducing the number of crashes at intersections, but it showed that FYA was a promising display technique.

Similar to the North Carolina study, a paper by researchers at Texas Southern University evaluated the safety of FYA installations at signals with PPLT phasing at 17 intersections in Tyler, Texas and Kennewick, Washington, using the EB method (11). This research specifically focused on intersections that operated in PPLT mode before and after the conversion, with the only change being in the change in the permissive display for the signal. A greater amount of data was analyzed with this study, as compared to the previously mentioned study, with four to six years of before data and one to two years of after data being available for analysis. Similar techniques were used to analyze the crash data, with the exception being that the SPFs were based on the number of left-turn lanes, AADT, and posted speed. The investigation indicated that overall, FYA did not decrease safety, although three of the 17 intersections had ORs greater than 1.0. The intersections with safety degradations were further analyzed and found that there were side-effects of the FYA installations at these sites, mostly due to higher volume to capacity ratios and specifics in signal timing. The two side-effects of the FYA were classified as a "red trap" problem and a "yellow sneakers" problem. They described the red trap as driver confusion during the steady yellow arrow portion of the left-turn after the protected portion - the researchers claimed that drivers misinterpreted the steady yellow for a flashing yellow indication, assuming they had to yield to oncoming traffic. The yellow sneakers issue was defined as vehicles executing left-turns and those who were in the opposing through direction speeding up to make it through the yellow indication concurrently - this was only observed when 
the two movements ended at the same time. These two crash types are illustrated in Figure 1 below as $\mathrm{C} 1$ and $\mathrm{C} 2$ from the study. As a result, the researchers suggested that the following additional changes be made when installing FYA for PPLT: allow for a longer clearance interval (red time after the protected portion ends) for confused drivers to clear the intersection, and offset the ending times of opposing left-turn movements in lead-lag PPLT operations.

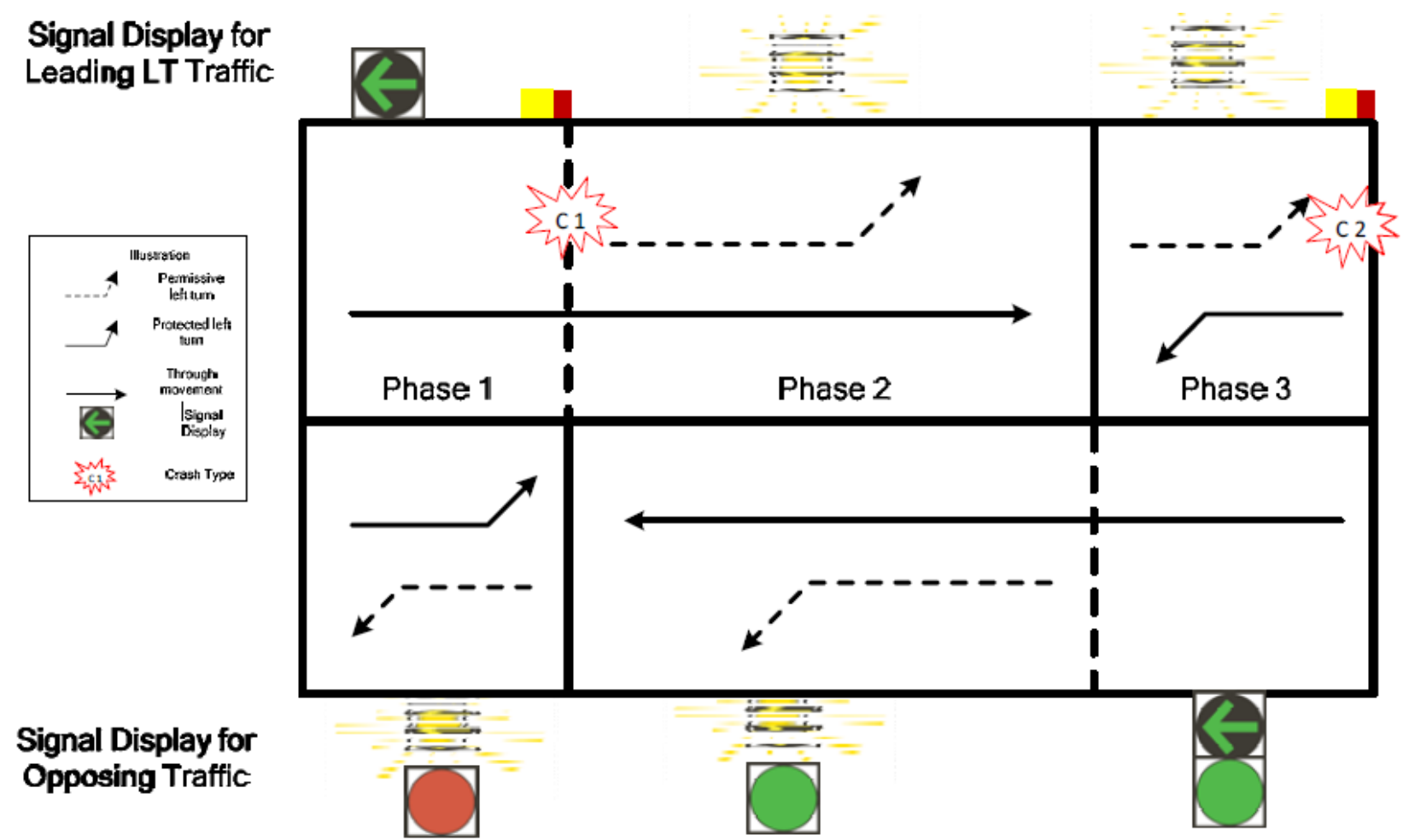

Figure 1 - FYA Side-Effects (11)

Another report examined the impacts of supplemental signage, evaluated FYA's impact on older and younger drivers, and conducted a cost-benefit analysis for the FYA installations (1). The research utilized both the naïve before and after and EB methods to analyze crashes at 86 intersections in Illinois that underwent a conversion from PPLT to PPLT-FYA and develop CMFs and their respective confidence intervals. From the safety analysis, CMFs were determined to be $0.63,0.61,0.71$, and 0.71 for left-turn related crashes, left-turn related crashes at intersections 
with supplemental signage, left-turn opposing through crashes, and left-turn opposing through crashes at intersections with supplemental signage. It should be noted that the researchers defined left-turn opposing through crashes as crashes that involved a left-turning vehicle and an opposing through direction vehicle. This indicates a $37 \%$ and $29 \%$ reduction in the target crash type, and that supplemental signage installed with FYA provided a slight safety improvement. Additionally, the researchers determined that the FYA installations had no effect on older drivers and that FYA helped younger drivers understand the PPLT phasing. For the cost-benefit analysis, the study compared crash cost reduction benefits to the initial cost of the FYA installation with a $3 \%$ discount rate over the 15 -year economic life of the signal. From this, it was determined that there was a 19.8 to 1 benefit to cost ratio, indicating that the conversion was economically justifiable. As a result of the study, the researchers produced accurate CMFs for target crash types and suggest that FYA continue to be implemented in place of standard green ball indications, as the FYA improves safety and is economically advantageous.

As another metric in determining the safety effectiveness of FYA deployments, a study from the University of South Florida examined gap acceptance as a short-term safety measure (12). Since the EB method requires years of after crash data to produce accurate results, the safety implications of FYA installations cannot be immediately studied to indicate whether the deployment was effective at a particular location. Because of this, the research developed a method of analyzing intersections with the upgrade using one month of before and two months of after data. Turning movement counts, conflicts (with the time of conflict recorded), and gap acceptance data were collected through video collection strategies. The study team concluded from the research that a slight reduction in crash risk was observed from the study site since the crossing tolerance, defined as the "time between the moment the turning vehicle clears the gap 
reference point and the moment the front bumper of the opposing vehicle touches such reference point," increased. Additionally, the study found that capacity improvements of up to $10 \%$ can be realized for moderate opposing traffic since drivers accepted shorter gaps.

\subsubsection{Phasing Changes and Flashing Yellow Arrow Installations}

To quantify the effects of FYA and PPLT conversions, a 2012 report determined CMFs for the changes from permissive only to PPLT and for the implementation of PPLT-FYA at intersections with permissive-only and protected-only prior control modes, separately (13). Using the EB method for the conversion from permissive to PPLT and a modified version of the EB method for the implementation of FYA, 71 sites in Toronto and North Carolina were analyzed for the change from permissive to PPLT, and 51 sites in Washington, Oregon, and North Carolina were analyzed for the installation of FYA. From the research, statistically significant CMFs were determined for many different groupings of parameters and crash types, including a value of 0.86 for left-turn crashes for the conversion from permissive to PPLT, and values of $1.34,2.24,0.75$, and 0.64 for total and left-turn crashes for converting from protected only to PPLT with FYA and total and left-turn crashes for converting at least one leg from permissive only to PPLT with FYA, respectively. In general, the study found that converting from permissive only to PPLT reduced crashes by $14 \%$ for the target crash type and the addition of FYA helped further reduce crashes, with a decrease in crashes by a total of $36 \%$. Additionally, converting from protected only to PPLT with FYA increased crashes by $124 \%$ for the target crash type.

A 2015 paper also developed CMFs for the conversion to FYA, with statistically significant results for conversions from five-section PPLT with green ball indication to PPLT 
with FYA and for conversions from permissive only with green ball indication to permissive only with the FYA indication (2). Additionally, the study attempted to determine CMFs for the conversion from permissive only to FYA PPLT, protected only to FYA PPLT, and protected only to FYA PPLT with time-of-day operation, but the results showed too much variability, preventing the CMFs from being statistically significant. For the statistically significant CMFs, sites from North Carolina were analyzed using a simple before and after method, utilizing the Highway Safety Manual's (HSM) SPFs for urban and suburban intersections to account for traffic volume variability. For the conversion from PPLT to FYA PPLT, statistically significant CMFs were found on the approach level at $0.93,0.85,0.78$, and 0.68 for total, injury, target (left-turn, same roadway), and target injury crash types, respectively for intersections with all legs that were treated receiving the same treatment. The conversion from permissive only to FYA permissive on an approach level only found statistically significant results of $0.69,0.41$, and 0.31 for injury, target, and target injury crash types, respectively. From the study, a reduction in crashes is evident for the PPLT to FYA PPLT conversion, and a more prominent reduction is evident for the permissive to FYA permissive conversion, although these results are not based on the EB method, which accounts for regression to the mean (RTM) bias.

Table 1 summarizes significant CMFs developed in past studies. 
Table 1 - Summary of Existing CMFs

\begin{tabular}{|c|c|c|c|c|}
\hline Study & CMF & Crash Type & Conversion & Notes \\
\hline \multirow{4}{*}{$\begin{array}{l}\text { Schattler } \\
\text { et al., } \\
2016 \text { (1) }\end{array}$} & 0.62 & Left-turn Related & \multirow{4}{*}{$\begin{array}{l}\text { PPLT to } \\
\text { PPLT-FYA }\end{array}$} & - \\
\hline & 0.71 & $\begin{array}{l}\text { Left-turn Opposing } \\
\text { Through }\end{array}$ & & - \\
\hline & 0.59 & Left-turn Related & & \\
\hline & 0.71 & $\begin{array}{c}\text { Left-turn Opposing } \\
\text { Through } \\
\end{array}$ & & With supplemental signage \\
\hline \multirow{5}{*}{$\begin{array}{l}\text { Srinivasan } \\
\text { et al., } \\
2012 \text { (13) }\end{array}$} & 0.86 & Left-turn & Perm to PPLT & - \\
\hline & 0.75 & Total & \multirow{2}{*}{$\begin{array}{l}\text { Perm to } \\
\text { PPLT-FYA }\end{array}$} & - \\
\hline & 0.64 & Left-turn & & - \\
\hline & 1.34 & Total & \multirow{2}{*}{$\begin{array}{c}\text { Protected-Only } \\
\text { to } \\
\text { PPLT-FYA } \\
\end{array}$} & - \\
\hline & 2.24 & Left-turn & & - \\
\hline \multirow{7}{*}{$\begin{array}{l}\text { Simpson } \\
\text { \& Troy, } \\
2015 \text { (2) }\end{array}$} & 0.93 & Total & \multirow{4}{*}{$\begin{array}{l}\text { PPLT to } \\
\text { PPLT-FYA }\end{array}$} & \multirow{2}{*}{ Approach-level } \\
\hline & 0.85 & Injury & & \\
\hline & 0.78 & Target & & \multirow{2}{*}{$\begin{array}{l}\text { Approach-level; Target = } \\
\text { left-turn, same roadway }\end{array}$} \\
\hline & 0.68 & Target/Injury & & \\
\hline & 0.69 & Injury & \multirow{3}{*}{$\begin{array}{l}\text { Perm to } \\
\text { Perm-FYA }\end{array}$} & - \\
\hline & 0.41 & Target & & - \\
\hline & 0.31 & Target/Injury & & - \\
\hline
\end{tabular}

* Protected-only phasing is indicated by PO, and permissive-only phasing is indicated by Perm.

All CMFs presented are statistically significant at the $95 \%$ confidence level.

\subsection{OPERATIONAL EFFECTS OF PPLT}

Another study on the operational impacts of PPLT phasing was conducted by the University of Central Florida (14). This research determined parameters that influence the number of left-turns that can be taken during the permissive phase of PPLT phasing, with a focus on comparing intersections with two or less opposing through lanes with intersections with more than two opposing through lanes. The purpose of this research was to further the knowledge of 
variables affecting permissive left-turns, rather than to provide specific guidelines for engineers. This was achieved by collecting and processing video data at six intersections near Orlando, Florida and creating regression models. Three different models were developed representing large intersections, small intersections with commercial land use, and small intersections with residential or school land uses. In the smaller intersection models, the single-factors of speed and lane use were significant, but were not significant in the larger intersection model. Additionally, the larger intersection model had total number of lanes crossed multiplied by the total opposing volume as a significant two-way factor, and the small intersection models had speed multiplied by the total opposing volume as a significant two-way factor. The factors that were common between the models were: permitted green time multiplied by the total left-turns, permitted green time multiplied by the number of lanes crossed, time-of-day, total number of lanes crossed, permitted green time, and total opposing volume. As the researchers concluded, the larger and small intersection models were statistically different, and the larger intersection model emphasized the number of lanes crossed, rather than speed (as the researchers stated that there are lower speed variations with these types of intersections). Additionally, as hypothesized, the stop control delay for protected only left-turn phasing was higher than that of PPLT phasing.

\subsection{COMBINED OPERATIONAL AND SAFETY EFFECTS OF PPLT}

As evident by the literature up to this point, PPLT can provide an operational benefit over protected only left-turn phasing at the cost of a reduction in safety, and that FYA provides operational, safety, and driver understanding benefits over other forms of permissive indications. All of the studies reviewed thus far have analyzed these impacts individually, with only a few touching on both safety and operational impacts of PPLT phasing. In an attempt to address both 
issues, a 2010 study by Texas Southern University created an approach to estimate both operational benefits and safety costs associated with PPLT phasing (15). The research developed models estimating left-turn delay for protected only and PPLT phasing modes using variables such as saturation flow of permissive and protected left-turn phases, as well as left-turn volumes and signal timings. For the cost analysis, the number of potential conflict gaps was estimated based on Poisson arrivals. To evaluate the benefits and costs simultaneously, dollar amounts per vehicle were assigned to the delay and conflict costs. These values were multiplied by their respective delay reduction and conflict predictions, and summed to determine the benefit or cost of a particular installation. By using this method, traffic engineers can evaluate whether or not installing PPLT will provide a safety and operational benefit, though the study only used one city for data collection and model validation, thus the methodology may not be accurate for other localities.

\subsection{EXISTING LEFT-TURN PHASING GUIDELINES}

The University of Kentucky's Transportation Research Program, in cooperation with the Federal Highway Administration (FHWA), developed recommendations in 1985 for when permissive phasing, including PPLT, should not be used based on an analysis of crashes at 58 intersections in Kentucky (16). Additionally, the study evaluated the characteristics of the related crashes. Both analyses utilized before and after crash data, with one to seven years of before, and a minimum of one year of after data. The study used a simple crash rate (number of crashes per year) as a metric to compare the before and after conditions following conversions from protected only (PO) to permissive left-turn phasing. From the analyses, the study found that left-turn crashes increase with permissive-only phasing, a dramatic increase in left-turn and 
total crashes were found at sites with speed limits over $45 \mathrm{mph}$, and that the overall severity of the incidents (except fatal severities) were higher than the statewide average. As a result of the study, it was recommended that any form of permissive phasing, including PPLT, should not be used when the speed limit is greater than $45 \mathrm{mph}$, the speed limit is over $35 \mathrm{mph}$ and protectedonly phasing currently exists, the left-turn movements must cross three or more opposing through lanes, there are dual left-turn lanes, there is not enough sight distance, or a left-turn crash issue exists, as result of a traffic conflicts study. All of the above guidelines applied to permissive-only and PPLT phasing modes, though the study only evaluated results of intersections converted from protected-only to permissive-only, therefore PPLT generalization may not be appropriate.

To address the gaps left in the 1985 study, the University of Kentucky's Transportation Center published a report to update guidelines for determining left-turn phasing, with an emphasis on high speed areas (17). In this study, 264 intersections, with a total of 518 approaches that underwent signal conversions, were evaluated using crash history, traffic conflict data, and operational characteristics. As with the previous study, the average number of crashes were compared in the before and after periods for each intersection, with the exception that the peak hour volumes were used as a method of exposure to weigh the crashes. Additionally, simulation models were used to determine left-turn delay, based on different variations of leftturn volumes, opposing volumes, cross street volumes, and number of approach lanes, as well as left-turn phasing (protected only, permissive, or PPLT). From the study, the researchers made a number of recommendations, the most prevalent being that PPLT phasing is preferred over protected-only phasing for lower left-turn delay, unless there is an existing or potential for a crash problem with left-turning vehicles, as defined by the following: four or more left-turn 
crashes in one year, six or more in two years, or eight or more in three years on the analysis approach. If the values are higher than these thresholds, crash rates should then be compared to rate tables developed by the study, based on different combinations of speed limits and number of opposing lanes. The researchers also established that if the product of left-turning and opposing volumes for the analysis hour exceeds 100,000 on a four-lane road or 50,000 on a twolane road, left-turn phasing should be considered (i.e. non-permissive). If the product exceeds 300,000 on a four-lane street or 150,000 on a two-lane street, protected-only phasing should be considered. Additionally, they determined that if the left-turn experiences 2 vehicle-hours or greater of delay during the peak hour, left-turn phasing should be considered.

Several years after the aforementioned study, the Transportation Research Board (TRB) published National Cooperative Highway Research Program (NCHRP) Report 457 that included formal guidance for left-turn phasing selection (18). In the report, the NCHRP stated that "twophase operation with permitted left-turn movements should be considered as a "starting point"' and that "left-turn phasing should only be provided if it will improve operations or safety." Additionally, they provided a flowchart for selection of left-turn phasing alternatives based on crash history, sight distance, site geometry, vehicular volume per cycle, and left-turn delay or cross product of volume (CPOV). The flow chart is provided in Figure 2 for reference. One major shortcoming to this guidance is that it only considers the peak period of the intersection being analyzed, i.e. whichever phasing is warranted for the peak hour should be applied for the entirety of the signal. Because of this, time-of-day phasing is not considered. 


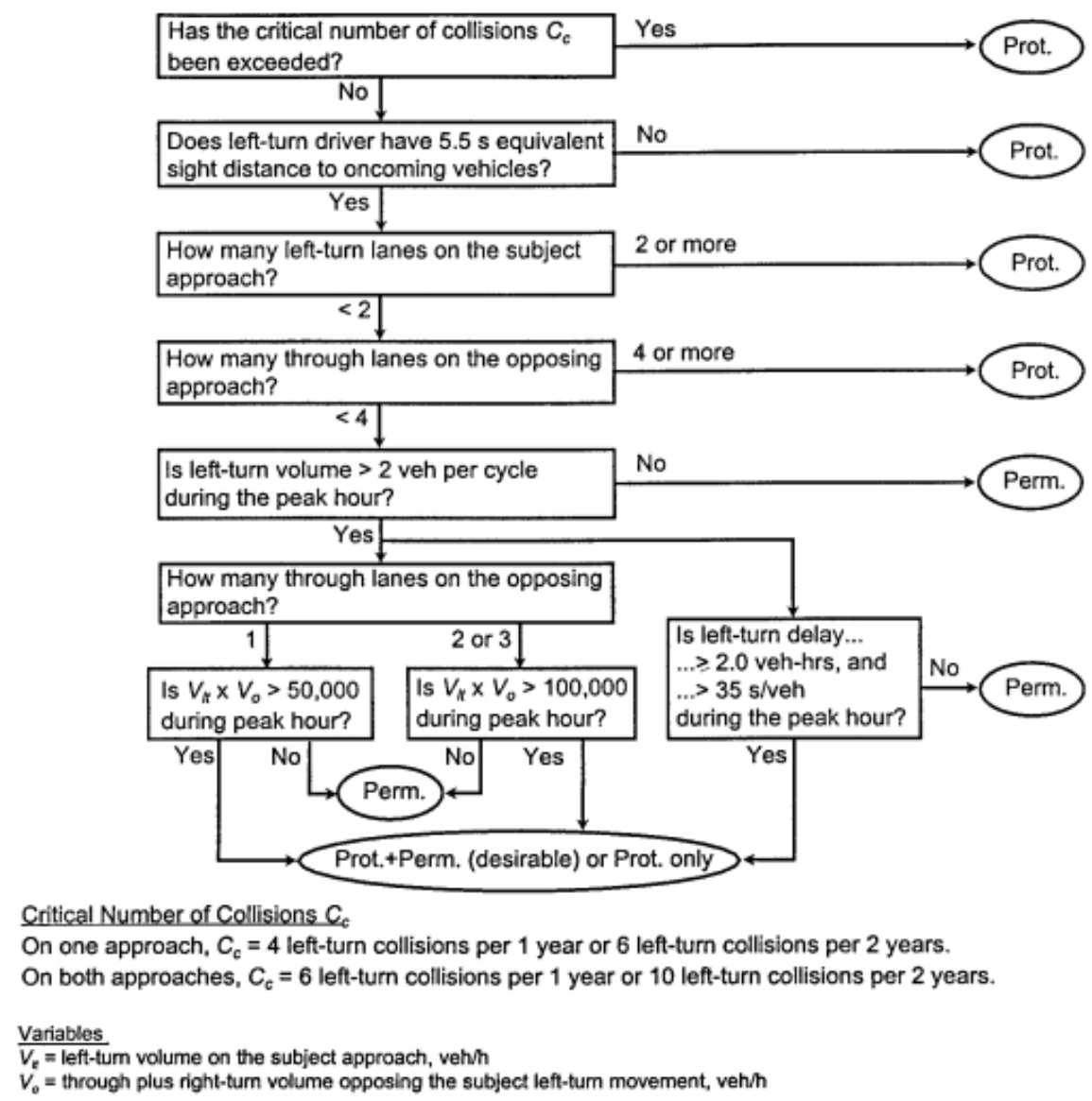

Figure 2 - NCHRP Report 457 Left-turn Phasing Flow Chart (18)

The University of Hawaii at Manoa examined the operational effects of PPLT phasing (19). In this study, researchers used simulations to develop regression models for volume and delay warrants for protected only or PPLT phasing. The significant factors determined by the analysis for left-turn volume thresholds were opposing through volume, left-turn volume on the cross street, and through and right volume on the cross street. The resulting model had an Rsquared value of 0.952 and standard error of 27.7 vehicles per hour. Additionally, it was found that when determining the cross product of volume (CPOV) threshold, values varied greatly. Thus, the researchers concluded that this was not a good left-turn phase criterion for the purpose of the study. The findings of the study were that left-turn warrants rely heavily on opposing 
through volumes and a decision tree was developed, using a similar structure to that of previously developed decision trees, with the addition of the regression model step. The flowcharts developed by this study can be seen in Figure 3 and Figure 4 .

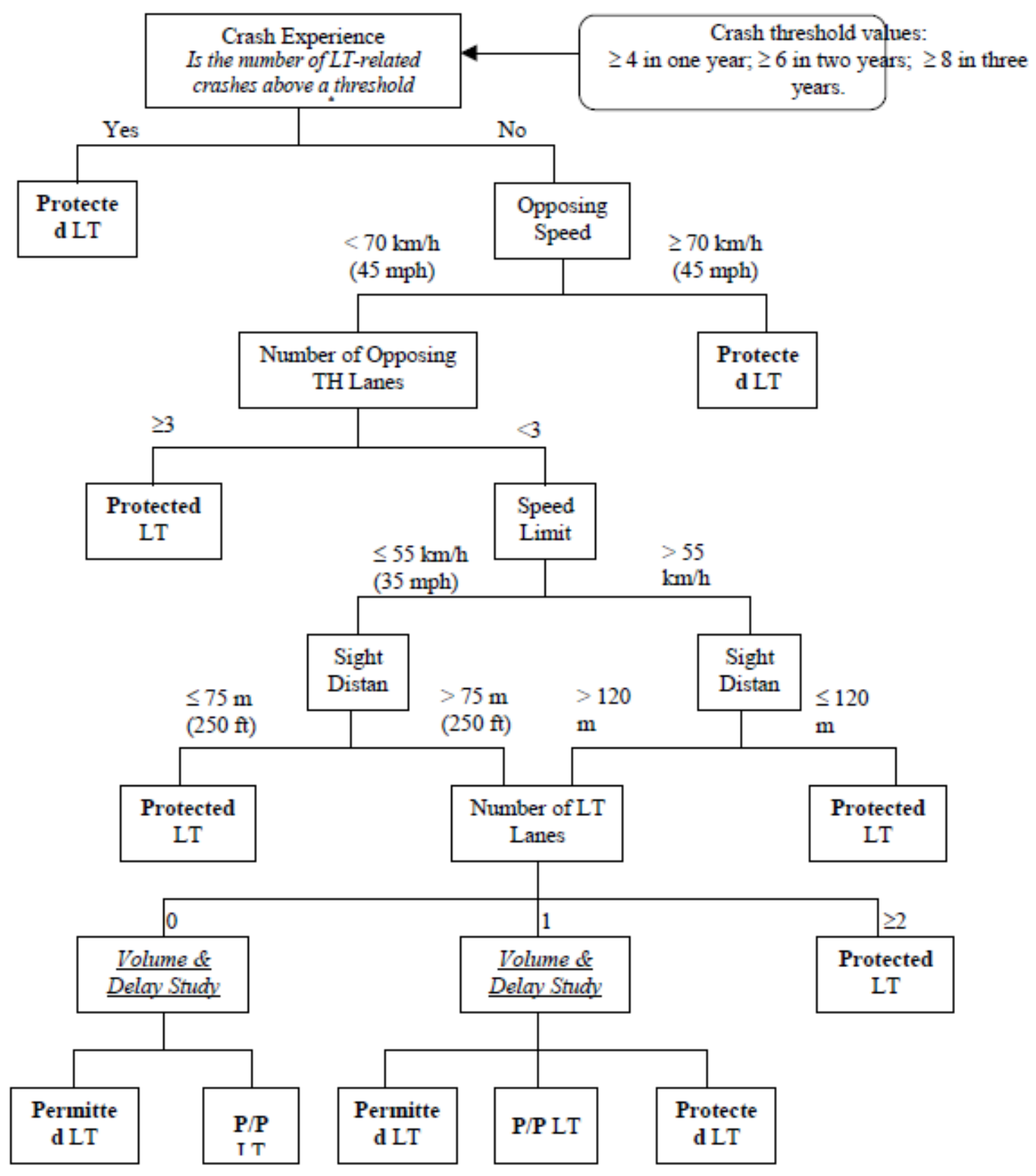

Figure 3 - University of Hawaii Left-turn Phasing Flowchart (19) 


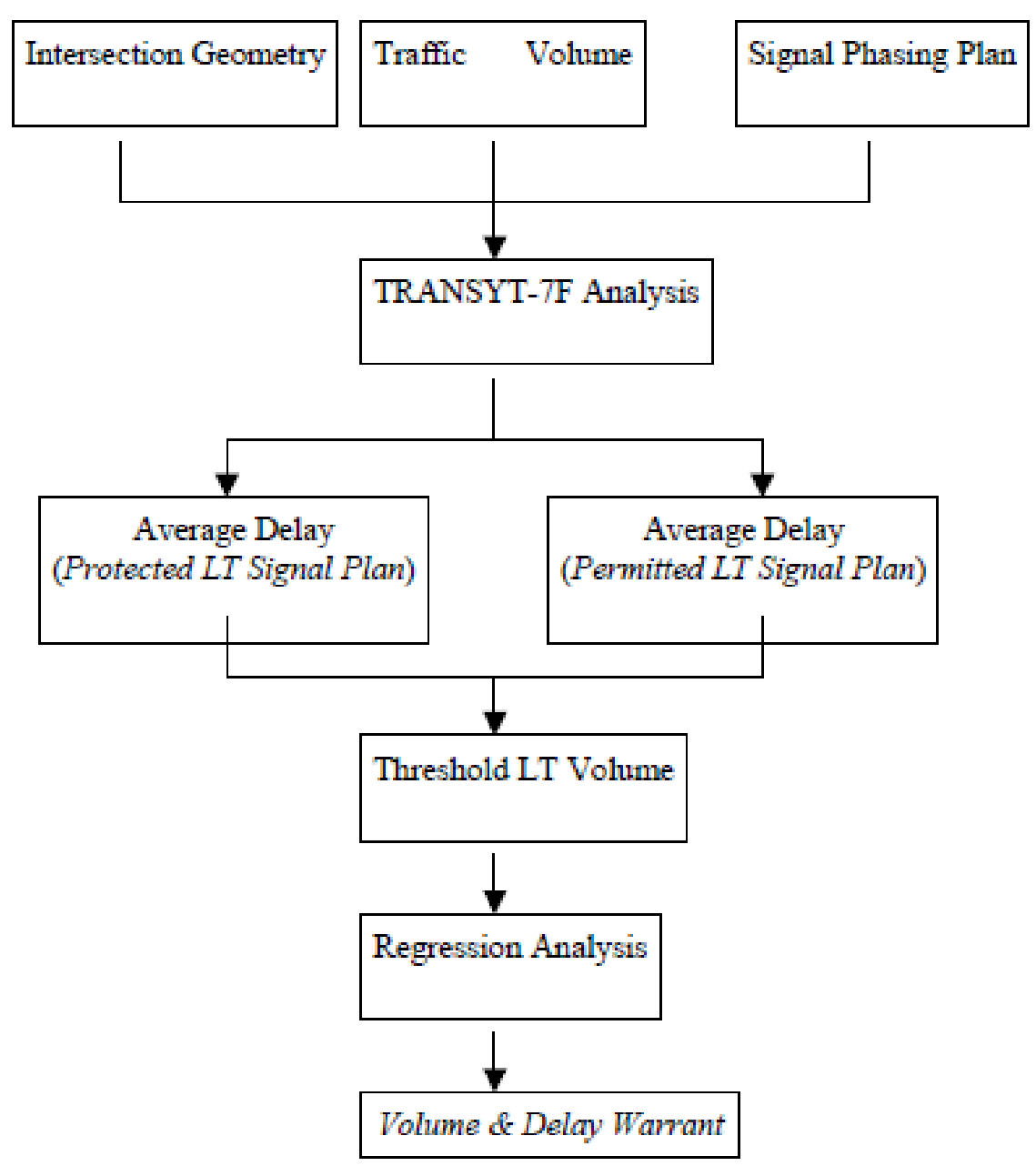

Figure 4 - University of Hawaii Left-turn Phasing Model Flowchart (19)

To provide additional formal guidance to the deployment of PPLT phasing and FYA signal indications, the FHWA included left-turn display and phasing sequence options sections in their 2008 Signal Timing Manual (20). In the document, it is stated that PPLT phasing can offer a good compromise between the safety benefits of protected only phasing and the efficiency benefits of permissive left-turn phasing. Additionally, they included guidelines for selecting leftturn phasing, including a flowchart similar to that of the one included in the NCHRP Report 457, shown in Figure 5. With regards to display, the manual suggests that the FYA permissive display be used for PPLT phasing to eliminate the "yellow trap" and allow for permissive left-turns 
during opposing protected left-turns. Also, the document indicated that NCHRP Project 3-54 concluded that the FYA indication was the best alternative to the green ball permissive signal display. 


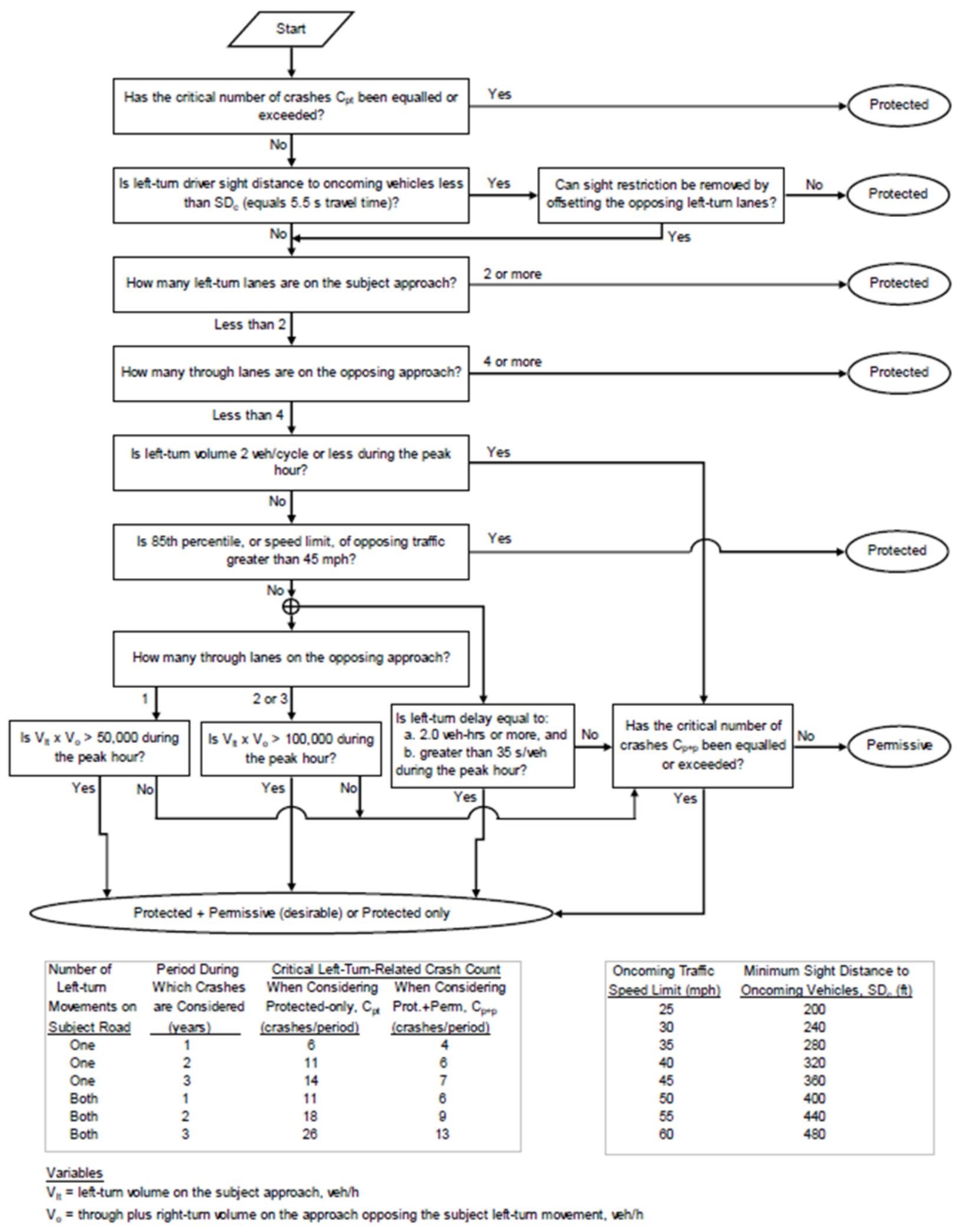

Figure 5 - FHWA Signal Timing Manual Left-turn Phasing Flowchart (20) 
To better put past and current research into context and practice, agencies and researchers have developed guidelines for the installation of FYA and PPLT at new and existing traffic signals. One study by the Texas Department of Transportation created a comprehensive set of guidelines for their state in 2009 (21). Safety and operational impacts of phasing mode, sequence, and display were analyzed in the study using detailed data for 26 intersections in Texas for the operational study and 111 pairs of intersection approaches for the safety study. For the operational impact study, GPS probe data for travel time and video traffic data were collected and used for simulation model development and validation. The models used a three-intersection roadway network to simulate both intersection and network impacts. Additionally, the delay and CPOV for the simulations were used as operational metrics for the intersections. In the safety impact study, data was used to develop regression models to determine left-turn crash frequency based intersection geometrics, signal control, signal display, and traffic conditions. The study also performed a before and after study using the EB method to determine ORs for intersections converted from protected only to PPLT phasing. As a result of the operational and safety studies performed the researchers, it was determined that the CPOV should be used as a volume-based criterion in determining phasing mode, and a phasing mode selection flowchart was created based on previous literature and the developed volume-based criteria, as shown in Figure 6. Additionally, the Department recommended that FYA can be used as an alternative to green ball permissive indication for PPLT, but should be installed on a region-wide basis. 


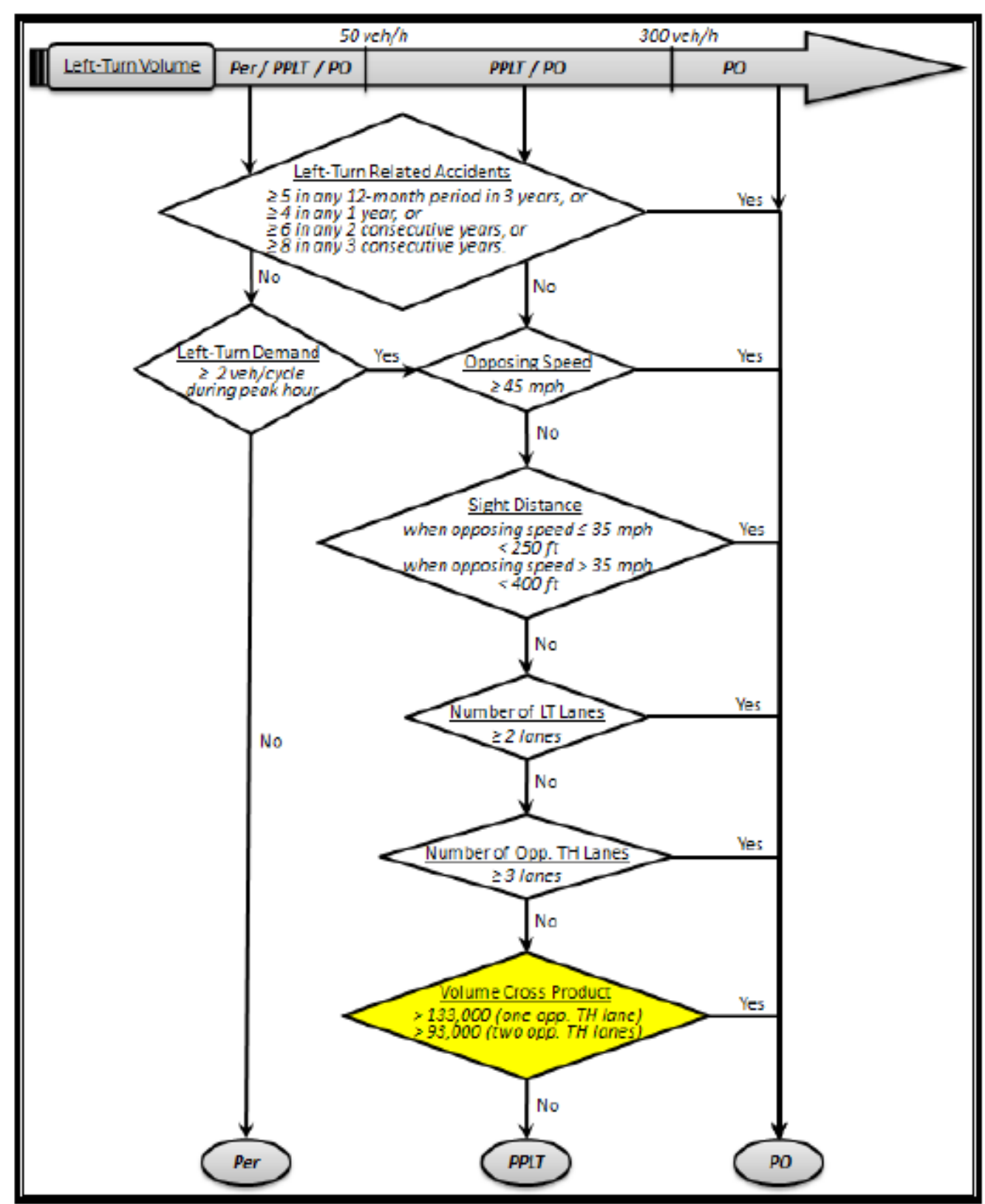

Note: "Per" means permissive mode; "PPLT" means protected/permissive mode; "PO" means protected-only mode.

Figure 6 - TxDOT Left-turn Phasing Flowchart (21)

Two reports by the Florida Department of Transportation and the University of Central Florida created a decision support system and time-of-day recommendations for the implementation of variable left-turn modes using FYA (22) and (23). In both papers, over 200 hours of processed data from intersections in Central Florida using video collection units was 
used to develop and validate the prediction models. Data that was collected from the video collection units included gap and volume analysis. Additionally, categorical data including intersection geometry was collected to create the models. In the 2013 study, the researchers developed a decision support system based on a generalized linear model, with input variables of time-of-day, number of crossing lanes, speed, permitted green time, total left-turn volume, total opposing volume, criteria (urban, rural, ramp, etc.), and land use. Of these variables, total leftturn volume and total opposing volume seemed to be the most significant factors, supporting the previously accepted notion that CPOV was a sufficient selection variable for left-turn phasing. The model developed by the researchers predicted the left-turn volume of the permitted portion of the phase. The system would then output the phasing recommended (either permissive or protected-only) and the number of left-turns and percentage of left-turns provided by the left-turn phasing by comparing the calculated permitted left-turn index (defined as permitted left-turn volume multiplied by total opposing volume divided by permitted green time in seconds) and permitted left-turn ratio (defined as the permitted left-turn volume divided by the total left-turn volume) to threshold values for one or two opposing lanes. This was done to determine if permissive phasing was feasible at a given intersection.

The 2017 study improved on the previous report by modeling delay, rather than the number of left-turns made during the phase. A stepwise regression approach was used to develop two models; one to predict the delay of either PPLT or permissive only phasing and a second to predict delay for PPLT or protected only. The study then developed graphs for one and two opposing lanes for the percent reduction in delay vs the percent left-turn index (determined by multiplying the ratio of permissive left-turn volume to total left-turn volume by the ratio of opposing volume during the permitted phase to the total opposing volume and dividing by the 
percentage of permitted green time in an hour), which can be found in Figure 7 and Figure 8. These graphs were used to decide if protected only or PPLT phasing should be provided for a given hour. A threshold for the percent reduction in delay was determined by the literature to be $10 \%$, which corresponds to a percent left-turn index of 92.4 for two opposing lanes and 71.5 for one opposing lane. These thresholds were used with the left-turn delay models produced to determine left-turn phasing. The model produces left-turn delay, which could then be used to calculate the percent reduction in delay for the intersection and the percentage left-turn index. If the value of percentage left-turn index is lower than that of the threshold value, PPLT phasing should be provided. From both of the studies, decision tools were developed to aid traffic engineers in determining left-turn phasing at given intersections based on operational impacts.

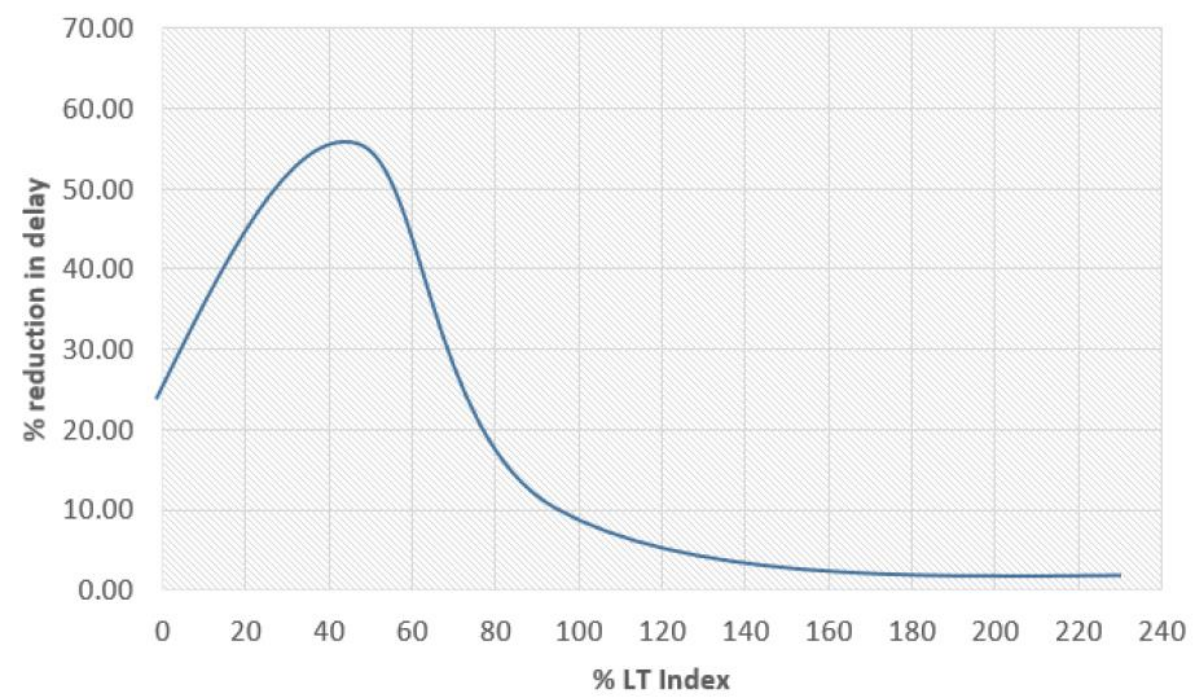

Figure 7 - Threshold for Two Opposing Lanes (23) 


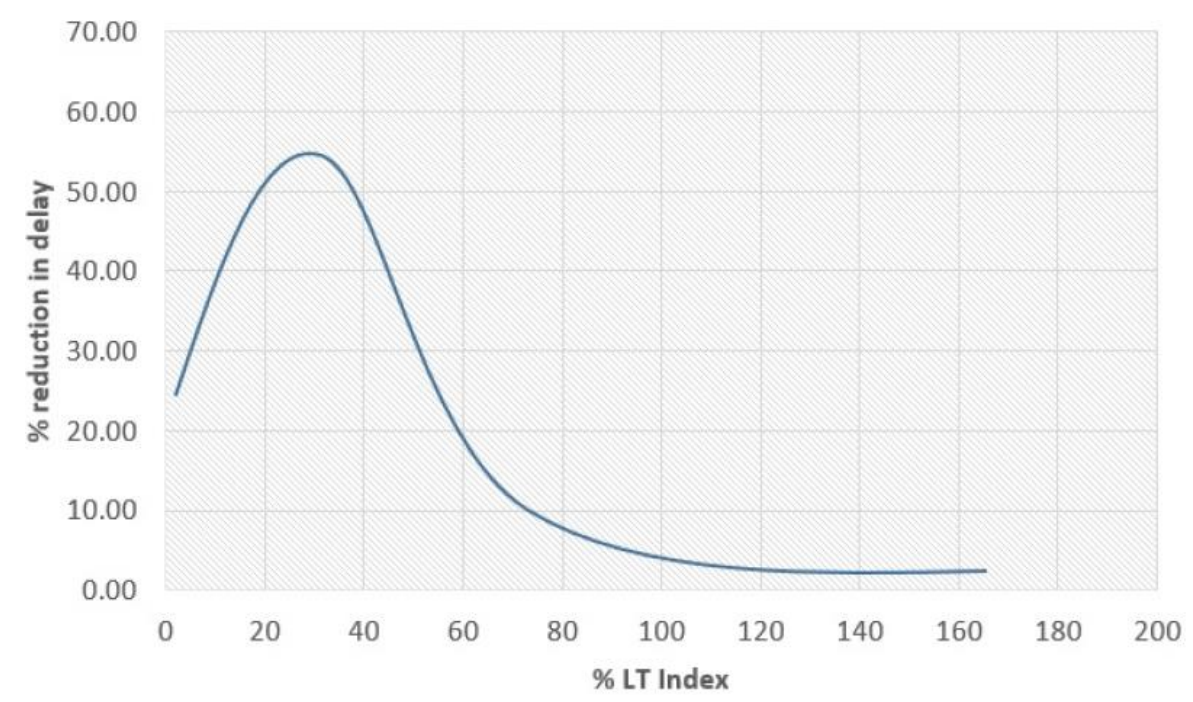

Figure 8 - Threshold for One Opposing Lane (23)

Finally, the Minnesota Department of Transportation developed guidelines for the timeof-day use of PPLT phasing by creating a spreadsheet tool based on relative crash risk predicted by statistical models (24). In the study, the researchers use a matched case-control technique to develop models based on geometric characteristics, crash data, and traffic volume data. Six models were developed to reflect different categories of intersections:

1. PPLT, opposing speed limit $<45 \mathrm{mph}$, no clear sight distance problem,

2. PPLT, opposing speed limit $<45 \mathrm{mph}$, possible sight distance problem,

3. PPLT, opposing speed limit $\geq 45 \mathrm{mph}$, possible sight distance problem,

4. PPLT, opposing speed limit $\geq 45 \mathrm{mph}$, no clear sight distance problem,

5. Permissive, opposing speed limit $<45 \mathrm{mph}$, possible sight distance problem,

6. Permissive, opposing speed limit $<45 \mathrm{mph}$, no clear sight distance problem.

The independent variables that were model inputs were hourly left-turn and opposing through volumes. In developing the model, researchers did not have turning movement counts for each day of the year, nor did they have hourly volumes for all times and days of the years, 
thus they developed methods of predicting values for days and times not gathered in the data collection. Five randomly sampled hours were selected per intersection (328 total intersections) to develop log linear models for the case-control design. The models produced were able to predict the relative risk of crashes through the 24-hour period selected. The models were also incorporated into a spreadsheet tool so that traffic engineers could view graphs of the relative risk and standard deviation throughout each hour of the day. The spreadsheet tool is provided in Figure 9 for reference. This study was successful in providing a tool to predict relative crash risk based on time-of-day, but it was developed in the Twin Cities area of Minnesota, therefore it may not be applicable to other locations.

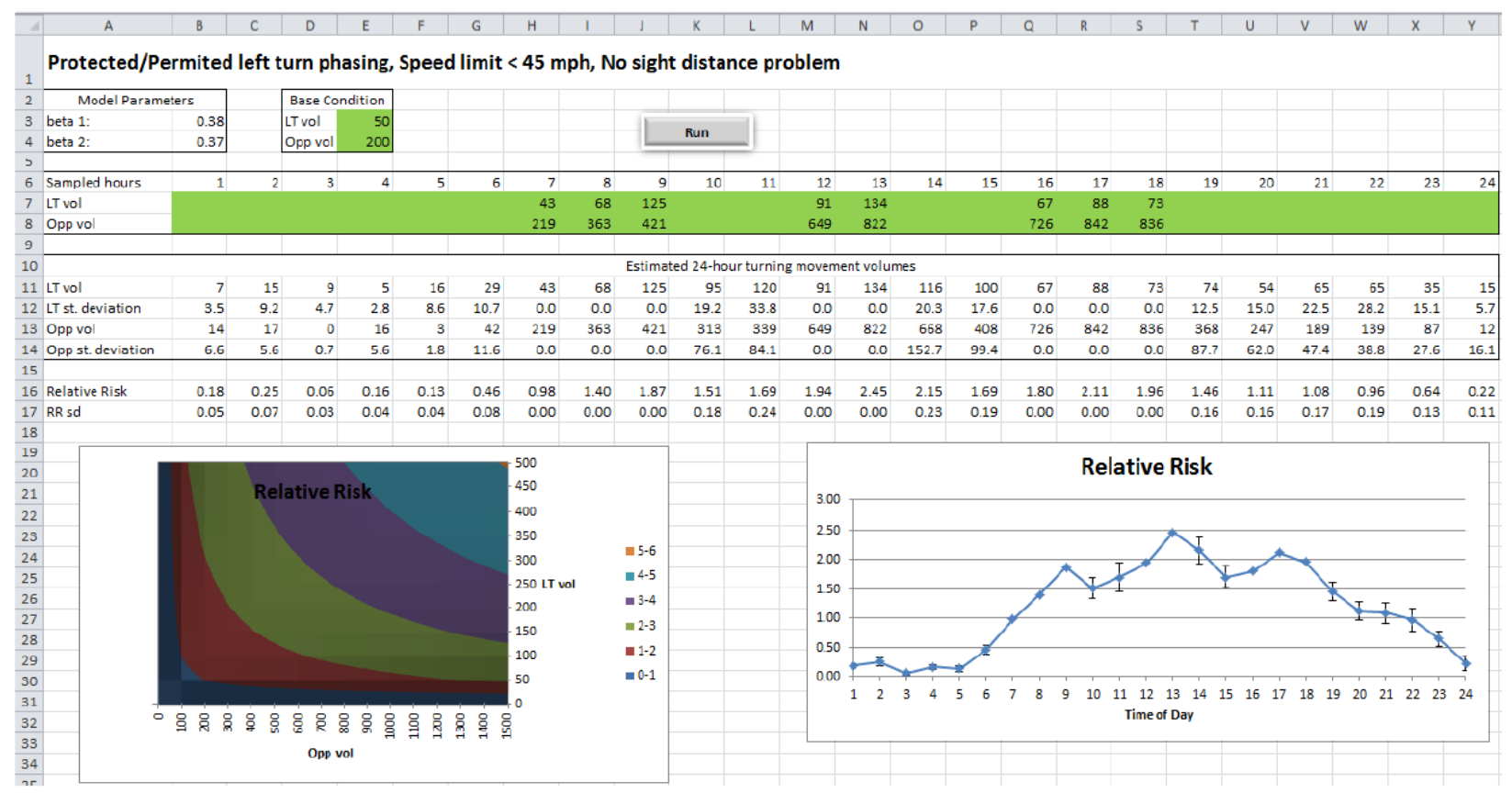

Figure 9 - MnDOT Relative Risk Spreadsheet Tool (24) 


\subsection{VIRGINIA DEPARTMENT OF TRANSPORTATION'S GUIDANCE ON LEFT- TURN PHASING SELECTION}

The Virginia Department of Transportation (VDOT) published a document in February 2015 that was mean to "equip traffic engineers in Virginia with the most appropriate tools to make informed and thoughtful decisions on left-turn phasing mode selection" (4). This document, as well as a previous study commissioned by VDOT, evaluated existing literature on guidance determination as well as the state-of-the-practice for state DOTs and VDOT regional guidelines throughout the state. A summary of the selection criteria for Protected-Only left-turn modes used by other state DOTs is included in Figure 10. Additionally, the document gave two critical evaluation questions that engineers should use when determining left-turn phasing: "from a safety perspective, can permissive left-turn movements be allowed on an approach?" and "should some level of left-turn protection (i.e. Protected/Permissive mode) be provided for efficiency reasons?" These questions should be constantly considered when determining phasing mode choice. Additionally, VDOT provides factors that should be considered in determining left-turn phasing mode, including sight distance, intersection geometry, critical crossing gap, and correctable left-turn crashes. For the critical crossing gaps criteria, the Department states that a CPOV greater than 50,000 for any hour should consider PPLT, but does not establish an upper bound for PPLT using the metric, stating that PPLT can still be considered in high CPOV conditions. Additionally, the document states that in evaluating correctable left-turn crashes, the EB method should be used, but crash rate can also be considered with AADT to account for exposure. In closing, the VDOT states that phasing selection choice should be considered on a site-by-site basis, and no fixed thresholds for certain phasing modes are provided. Also, sight distance and critical crossing gap should be the first factors considered in reviewing 
intersections. 
Summary of Criteria Used in Selection of Protected-Only Mode, by State*†

\begin{tabular}{|c|c|c|c|c|c|c|c|c|c|c|}
\hline & $\begin{array}{l}\text { Left Turn } \\
\text { Volume }\end{array}$ & $\begin{array}{l}\text { Crash } \\
\text { History }\end{array}$ & $\begin{array}{l}\text { Sight } \\
\text { Distance }\end{array}$ & $\begin{array}{l}\text { Number of } \\
\text { Left Turn } \\
\text { Lanes }\end{array}$ & $\begin{array}{l}\text { Opposing } \\
\text { Speed Limit }\end{array}$ & $\begin{array}{l}\text { Opposing } \\
\text { Thru Lanes }\end{array}$ & Intersection Geometry & $\begin{array}{l}\text { Pedestrian } \\
\text { Volume }\end{array}$ & $\begin{array}{l}\text { Left Turn } \\
\text { Demand }\end{array}$ & $\begin{array}{l}\text { Existing } \\
\text { Sequence }\end{array}$ \\
\hline$A Z$ & & $\begin{array}{c}\text { 4 / } 6 \text { LT crashes in one } \\
\text { year }(1 \text { app / } 2 \\
\text { opp. app); or } 6 / 10 \text { in } 2 \\
\text { years }\end{array}$ & $\begin{array}{c}\text { Limited sight distance due } \\
\text { to geometry or opposing } \\
\text { LT vehicles }\end{array}$ & $\begin{array}{c}\text { Mainline has } \\
2 \text { or more LT } \\
\text { only lanes }\end{array}$ & $\begin{array}{l}\text { Opposing speed } \\
\text { limit }>45 \mathrm{mph}\end{array}$ & $\begin{array}{l}3 \text { or more opposing } \\
\text { thru lanes }\end{array}$ & & & & \\
\hline GA & & $\begin{array}{c}5 \text { or more } \mathrm{LT} \text { crashes in } 2 \\
\text { years }\end{array}$ & Limited sight distance & $\begin{array}{c}\text { Mainline has } \\
2 \text { or more LT } \\
\text { only lanes }\end{array}$ & $\begin{array}{c}\text { Opposing speed } \geq \\
45 \text { mph AND } 3 \text { or } \\
\text { more opposing thru } \\
\text { lanes }\end{array}$ & $\begin{array}{c}3 \text { or more opposing } \\
\text { thru lanes AND } \\
\text { opposing speed } \geq 45 \\
\text { mph }\end{array}$ & $\begin{array}{l}\text { Unusual intersection } \\
\text { geometry }\end{array}$ & $\begin{array}{l}\text { High } \\
\text { pedestrian } \\
\text { volumes }\end{array}$ & & \\
\hline LA & & $\begin{array}{c}\text { 4 / } 6 \text { LT crashes in one } \\
\text { year }(1 \text { app / } 2 \\
\text { opp. app); or } 6 / 10 \text { in } 2 \\
\text { years }\end{array}$ & $\begin{array}{l}\text { Limited sight distance (see } \\
\text { table in following section) }\end{array}$ & $\begin{array}{c}\text { Mainline has } \\
2 \text { or more LT } \\
\text { only lanes }\end{array}$ & $\begin{array}{c}\text { Opposing speed } \geq \\
45 \text { mph AND } 3 \text { or } \\
\text { more opposing thru } \\
\text { lanes }\end{array}$ & $\begin{array}{c}3 \text { or more opposing } \\
\text { thru lanes AND } \\
\text { opposing speed } \geq 45 \\
\text { mph }\end{array}$ & $\begin{array}{l}\text { Intersection geometry } \\
\text { creates a conflicting left- } \\
\text { turn path }\end{array}$ & & & \\
\hline $\mathrm{Ml}$ & & $\begin{array}{c}4 / 6 \mathrm{LT} \text { crashes in any } \\
12 \text {-month period (1 app / } \\
2 \\
\text { opp. app); or } 6 / 10 \text { in } 2 \\
\text { years }\end{array}$ & $\begin{array}{c}\text { Limited sight distance due } \\
\text { to geometry or opposing } \\
\text { LT vehicles }\end{array}$ & & $\begin{array}{l}\text { Opposing speed } \\
\text { limit }>45 \mathrm{mph}\end{array}$ & $\begin{array}{l}3 \text { or more opposing } \\
\text { thru lanes }\end{array}$ & & & & \\
\hline $\mathrm{MN}$ & $\begin{array}{c}\text { Peak hour LT } \\
\text { volume }>250 \\
\text { vehicles or cross- } \\
\text { product }>80,000 ; \\
\text { AND speed limit } \geq \\
45 \text { mph }\end{array}$ & $\begin{array}{c}5 \text { or more LT crashes } \\
\text { over } 3 \text { years }\end{array}$ & $\begin{array}{l}\text { Mainline LT has limited } \\
\text { sight distance (per } \\
\text { AASHTO) }\end{array}$ & $\begin{array}{c}\text { Mainline has } \\
2 \text { or more LT } \\
\text { only lanes }\end{array}$ & & $\begin{array}{l}3 \text { or more opposing } \\
\text { thru lanes }\end{array}$ & $\begin{array}{l}\text { Intersection geometry } \\
\text { creates a conflicting left- } \\
\text { turn path }\end{array}$ & & & $\begin{array}{l}\text { Lead-lag sequence is } \\
\text { already in use }\end{array}$ \\
\hline OR & $\begin{array}{l}\text { LT volume }>300 \mathrm{vph} \\
\text { OR volume cross- } \\
\text { product }>150,00 / \\
300,000 \text { (1/ } 2 \\
\text { opposing lanes) }\end{array}$ & $\begin{array}{c}\text { LT crashes } \geq 5 \text { per } \\
\text { approach in any } 12 \text { - } \\
\text { month period in } 3 \text { years }\end{array}$ & $\begin{array}{l}\text { Limited sight distance (see } \\
\text { table in following section }\end{array}$ & $\begin{array}{c}\text { Mainline has } \\
2 \text { or more LT } \\
\text { only lanes }\end{array}$ & $\begin{array}{l}\text { Opposing speed } \\
\text { limit }>45 \mathrm{mph}\end{array}$ & $\begin{array}{c}3 \text { or more opposing } \\
\text { thru lanes }\end{array}$ & & & & $\begin{array}{l}\text { Lead-lag is required } \\
\text { for efficient operation } \\
\text { but a flashing yellow } \\
\text { arrow display cannot } \\
\text { be installed }\end{array}$ \\
\hline$T X$ & $\begin{array}{c}\text { Volume cross- } \\
\text { product }>133,000 \\
\text { (1 opp. thru lane) or } \\
>93,000 \text { ( } 2 \text { opp. } \\
\text { thru lanes) }\end{array}$ & $\begin{array}{l}\text { LT crashes } \geq 5 \text { in any } 12- \\
\text { month period in } 3 \text { years; } \\
\geq 4 \text { in any } 1 \text { year; } \geq 6 \text { in } \\
\text { any } 2 \text { consec. years; } \geq 8 \\
\text { in any } 3 \text { consec. years }\end{array}$ & $\begin{array}{l}\text { Opp. speed } \leq 35 \mathrm{mph} \text { and } \\
\mathrm{SD}<250 \mathrm{ft} \text {; or opp. speed } \\
>35 \mathrm{mph} \text { and } \mathrm{SD}<400 \mathrm{ft}\end{array}$ & $\begin{array}{c}\text { Mainline has } \\
2 \text { or more LT } \\
\text { only lanes }\end{array}$ & $\begin{array}{l}\text { Opposing speed } \\
\text { limit } \geq 45 \mathrm{mph}\end{array}$ & $\begin{array}{c}3 \text { or more opposing } \\
\text { thru lanes }\end{array}$ & & & $\begin{array}{c}\text { LT demand } \geq 2 \\
\text { vehicles per cycle } \\
\text { in peak hour AND } \\
\text { opposing speed } \\
\text { limit } \geq 45 \mathrm{mph}\end{array}$ & \\
\hline WA & & $\begin{array}{c}\text { LT crashes on any } \\
\text { approach } \geq 3 / \text { year or } \geq 5 \\
\text { in two consec. years }\end{array}$ & $\begin{array}{l}\text { Opp. speed } \leq 35 \mathrm{mph} \text { and } \\
\mathrm{SD}<250 \mathrm{ft} \text {, or opp. speed } \\
>35 \mathrm{mph} \text { and } \mathrm{SD}<400 \mathrm{ft} \text {; } \\
\text { AND peak hour LT volume } \\
\text { exceeds storage capacity }\end{array}$ & $\begin{array}{c}\text { Mainline has } \\
2 \text { or more LT } \\
\text { only lanes }\end{array}$ & $\begin{array}{c}\text { Opposing speed } \\
\text { limit }>45 \text { mph AND } \\
\text { peak hour LT } \\
\text { volume exceeds } \\
\text { storage capacity }\end{array}$ & $\begin{array}{l}3 \text { or more opposing } \\
\text { thru lanes (incl. RT } \\
\text { lanes) AND peak hour } \\
\text { LT volume exceeds } \\
\text { storage capacity }\end{array}$ & $\begin{array}{c}\text { Geometry or } \\
\text { channelization is } \\
\text { confusing AND peak hour } \\
\text { LT volume exceeds } \\
\text { storage capacity }\end{array}$ & & & \\
\hline
\end{tabular}

† Maryland is not included in this table because it has no statewide mode selection guidelines.

Figure 10 - Protected-Only Criteria by State (4) 


\subsection{SUMMARY AND GAPS IN EXISTING LITERATURE}

After reviewing current practices, guidelines, and evaluation techniques for PPLT-FYA intersections, there are several gaps in the literature. One of the limitations of the reports reviewed is the lack of PPLT-FYA CMFs developed for geometry-specific conditions, specifically the number of approaches at the intersection, and angle crash types. Through developing separate CMFs for three- and four-leg intersections, this research will add to the accuracy of CMFs already established by other studies. Intersection geometry is important to the CMFs, as separate analysis should be done for different types of sites so that more accurate reduction factors are used. With respect to angle crash type CMFs, this study developed these to provide practitioners with an easier way to evaluate target crash types. Since other forms of target crash type such as left-turn opposing through crashes require more specific data, crashes would need to be individually evaluated to determine if they meet the criteria, whereas crash type coding such as angle crashes are coded in most crash reports, allowing easier analysis by field staff.

Prior guidance on left-turn phase selection often focused on only operational or safety metrics, and typically assumed the same phasing mode would be used throughout the day. This study will create guidance based on both operational and safety impacts of left-turn phasing. By using simulation runs to estimate operations and simulations and CMFs to estimate safety, these outputs will be combined to create specific guidance that VDOT engineers can use to assess different phasing alternatives. Although some studies have set out to view both aspects of intersection phasing, most focused on either operations or safety ultimately, although they touch on the other aspect briefly through past research. Additionally, this research will allow for the evaluation of a left-turn signal in a time-of-day (TOD) operational mode, which most previous 
studies have not covered. 


\section{CHAPTER 3: SAFETY ANALYSIS OF PHASING AND PERMISSIVE LEFT-TURN DISPLAYS}

To develop CMFs for the conversion from PPLT to PPLT-FYA and CMFs for the conversion from protected-only to PPLT-FYA, a before and after study using the Empirical Bayes (EB) approach was used. For this method, analysis sites that underwent signal conversions were selected based upon completeness of AADT and crash data between 2008 and 2016. From this process, 37 sites were selected for analysis: 29 green ball to FYA sites and 8 protected-only to PPLT-FYA sites.

Once necessary data was collected and the analysis sites were selected, analysis of several sub-sets of the sites was performed to develop specific CMFs for combinations of crash types, crash severities, and intersection types. Additionally, the two conversion types were analyzed separately to illustrate the effects of the different treatments. The results from this analysis were then checked for statistical significance, and final CMF values were selected. These values were then compared to the numbers presented by studies mentioned earlier in this paper to identify if the CMFs were consistent with one another.

\subsection{METHODOLOGY}

\subsubsection{Before/After Study EB Method}

The analysis performed in this thesis used a before and after study using the EB method. By using the EB method, regression to the mean bias can be avoided so that the results are not affected by unusually high or low observed crashes for the years selected. The EB method circumvents this by using a combination of observed and predicted number of crashes to form an expected number of crashes for the before installation period for each site. Data requirements for 
the calculation process included Safety Performance Function (SPF) predicted crashes and fieldobserved crashes for each year of the analysis sites, as well as an overdispersion factor.

\subsubsection{Safety Performance Functions}

When using the EB method, Safety Performance Functions (SPFs) are used to predict the amount of crashes at a location. Since all sites were in Virginia, Virginia-specific SPFs were used in the calculations. These signalized intersection SPFs were developed by the Virginia Transportation Research Council in 2010 using generalized linear modeling with a negative binomial distribution (25). Of these, there are 32 SPFs for all combinations of urban and rural locations, three- and four-leg intersections, total and fatal and injury crash severities, and statewide and regional locations. All of the Virginia-specific SPFs use the same model form, with major and minor street average annual daily traffic (AADT) as predictor variables. Base conditions for the SPFs were the same as those in the Highway Safety Manual (HSM) (26). Each of the intersections in this study used the corresponding SPF for their region, location type, and intersection geometry. Of the 32 available Virginia intersection SPFs, 18 were utilized in this study, corresponding to the different intersection types and locations of the dataset. The parameters of these SPFs can be found below in Table 2. The equation form for the Virginiaspecific SPFs is shown below in Equation 1.

$$
N_{\text {predicted }}=e^{\alpha} * \text { Major } A A D T^{\beta 1} * \text { Minor } A A D T^{\beta 2}
$$


Table 2 - Parameters for SPFs Utilized (25)

\begin{tabular}{|c|c|c|c|c|c|c|c|}
\hline Region & $\begin{array}{c}\text { Crash } \\
\text { Severity }\end{array}$ & Urban/Rural & $\begin{array}{c}\text { Intersection } \\
\text { Legs }\end{array}$ & $a$ & $\beta 1$ & $\beta 2$ & $\mathrm{k}^{*}$ \\
\hline \multirow{6}{*}{ Northern } & \multirow{3}{*}{ All } & \multirow{2}{*}{ Urban } & 3-leg & -4.999 & 0.5555 & 0.1554 & 0.393 \\
\hline & & & 4-leg & -8.3067 & 0.7522 & 0.328 & 0.2119 \\
\hline & & Rural & 4-leg & -1.604 & 0.2284 & 0.1514 & 0.3211 \\
\hline & \multirow{3}{*}{$\begin{array}{l}\text { Fatal } \\
\text { and } \\
\text { Injury }\end{array}$} & \multirow{2}{*}{ Urban } & 3-leg & -7.3982 & 0.6496 & 0.2088 & 0.4152 \\
\hline & & & 4-leg & -9.6546 & 0.7603 & 0.3597 & 0.2056 \\
\hline & & Rural & 4-leg & -3.3285 & 0.3601 & 0.0597 & 0.2505 \\
\hline \multirow{8}{*}{ Western } & \multirow{4}{*}{ All } & \multirow{2}{*}{ Urban } & 3-leg & -9.6143 & 0.8677 & 0.3297 & 0.3719 \\
\hline & & & 4-leg & -12.3913 & 1.0631 & 0.4567 & 0.1624 \\
\hline & & \multirow{2}{*}{ Rural } & 3-leg & -6.4368 & 0.544 & 0.2863 & 0.4112 \\
\hline & & & 4-leg & -6.3951 & 0.5508 & 0.3106 & 0.1525 \\
\hline & \multirow{4}{*}{$\begin{array}{l}\text { Fatal } \\
\text { and } \\
\text { Injury }\end{array}$} & \multirow{2}{*}{ Urban } & 3-leg & -11.0104 & 0.908 & 0.3226 & 0.5043 \\
\hline & & & 4-leg & -11.4284 & 0.8662 & 0.4412 & 0.1492 \\
\hline & & \multirow{2}{*}{ Rural } & 3-leg & -8.8607 & 0.7059 & 0.2809 & 0.392 \\
\hline & & & 4-leg & -8.0583 & 0.6809 & 0.2557 & 0.2285 \\
\hline \multirow{4}{*}{ Eastern } & \multirow{2}{*}{ All } & \multirow{2}{*}{ Urban } & 3-leg & -6.7518 & 0.6157 & 0.2969 & 0.3343 \\
\hline & & & 4-leg & -8.8553 & 0.7825 & 0.3706 & 0.1346 \\
\hline & \multirow{2}{*}{$\begin{array}{l}\text { Fatal } \\
\text { and } \\
\text { Injury }\end{array}$} & \multirow{2}{*}{ Urban } & 3-leg & -7.266 & 0.5508 & 0.3107 & 0.2975 \\
\hline & & & 4-leg & -9.9582 & 0.7484 & 0.4017 & 0.1269 \\
\hline
\end{tabular}

$* \mathrm{k}$, the overdispersion parameter, is used in calculating the number of expected crashes

\subsubsection{Selection of Analysis Sites}

Before starting the analysis, appropriate sites had to be carefully selected to ensure the data needed for CMF calculations was available. Initially, 347 intersections in Virginia that had a planned or completed conversion to PPLT phasing with FYA permissive portion signal display from any other left-turn phasing and display were identified. A total 87 of these intersections were found to have already undergone the conversion, thus these sites were able to be used for 
EB analysis. Of these locations, 44 were determined to have complete AADT data between the analysis years. Instances where there were incomplete AADT data include when the intersection had at least one driveway as an approach (as VDOT does not maintain these segments or record their traffic count data) or there were years of missing counts on an approach, as explained in the next section of this chapter.

Each of the 44 locations had an initial left-turn phasing of either PPLT with green ball or protected-only with green arrow on the approaches and were converted to PPLT-FYA after the change. Because of this, only the conversions from PPLT to PPLT-FYA and protected-only to PPLT-FYA could be analyzed, although the original goal was to analyze more conversion types, such as permissive-only with green ball to PPLT-FYA. Upon further inspection of these locations, four were removed since their conversion dates could not be determined, one was removed as it was found to be unsignalized before the FYA implementation, and two were removed since they had commercial driveways in close proximity to them. As a result, 37 final intersections were selected for analysis: 29 signals converted from PPLT to PPLT-FYA and 8 signals converted from protected-only to PPLT-FYA. A summary of these intersections can be found below in Table 3, with the "Before Conversion" column representing the signal condition before the conversion to PPLT-FYA; PPLT represents green ball indication with PPLT phasing, and PO indicated protected-only phasing. Additionally, AADT and crashes per year values that are presented represent average values across the before and after periods. The majority of the PPLT to PPLT-FYA conversions occurred in the western part of the state, while protected to PPLT-FYA conversions occurred mainly in the northern and eastern parts of the state. 
Table 3 - Summary of Intersections for CMF Calculations

\begin{tabular}{|c|c|c|c|c|c|c|c|c|c|c|c|c|c|c|}
\hline \multirow{2}{*}{ Intersection } & \multirow{2}{*}{$\begin{array}{c}\text { Before } \\
\text { Conversion } \\
\end{array}$} & \multirow{2}{*}{ Region } & \multirow{2}{*}{$\begin{array}{l}\text { Area } \\
\text { Type }\end{array}$} & \multirow{2}{*}{$\begin{array}{l}\text { \# of } \\
\text { Legs } \\
\end{array}$} & \multicolumn{2}{|c|}{ CMF } & \multicolumn{2}{|c|}{ Years } & \multicolumn{2}{|c|}{ Major Road AADT } & \multicolumn{2}{|c|}{ Minor Road AADT } & \multicolumn{2}{|c|}{ Crashes/Year } \\
\hline & & & & & Before & After & Before & After & Before & After & Before & After & Before & After \\
\hline 1 & PPLT & Western & Urban & 4 & 0.49 & 0.49 & 5.0 & 1.9 & 19,883 & 22,461 & 7,400 & 7,346 & 9.6 & 8.0 \\
\hline 2 & PPLT & Western & Urban & 3 & 0.77 & 0.77 & 5.0 & 1.9 & 13,840 & 13,905 & 2,778 & 2,923 & 0.4 & 1.0 \\
\hline 3 & PPLT & Western & Urban & 3 & 0.87 & 0.87 & 5.0 & 1.9 & 9,554 & 9,341 & 4,062 & 4,169 & 1.2 & 1.0 \\
\hline 4 & PPLT & Western & Urban & 4 & 0.63 & 0.63 & 5.0 & 1.9 & 7,971 & 7,988 & 3,188 & 3,349 & 2.0 & 2.0 \\
\hline 5 & PPLT & Western & Rural & 4 & 0.48 & 0.48 & 5.0 & 1.9 & 8,812 & 8,703 & 2,288 & 2,490 & 3.6 & 1.0 \\
\hline 6 & PPLT & Western & Rural & 3 & 0.73 & 0.73 & 5.9 & 2.0 & 9,607 & 9,603 & 5,459 & 5,551 & 1.4 & 1.5 \\
\hline 7 & PPLT & Western & Rural & 3 & 0.82 & 0.82 & 5.9 & 2.0 & 3,767 & 4,175 & 3,549 & 3,793 & 1.5 & 1.5 \\
\hline 8 & PPLT & Western & Rural & 4 & 0.62 & 0.62 & 5.9 & 2.0 & 17,891 & 18,095 & 2,799 & 3,166 & 5.3 & 3.5 \\
\hline 9 & PPLT & Western & Rural & 3 & 0.79 & 0.79 & 5.5 & 1.4 & 5,878 & 5,837 & 2,284 & 2,420 & 1.0 & 0.0 \\
\hline 10 & PPLT & Western & Rural & 4 & 0.57 & 0.57 & 5.0 & 1.9 & 8,143 & 7,664 & 532 & 544 & 1.4 & 1.5 \\
\hline 11 & PPLT & Western & Rural & 4 & 0.55 & 0.55 & 5.0 & 1.9 & 4,388 & 4,442 & 3,880 & 4,018 & 0.8 & 0.5 \\
\hline 12 & PPLT & Western & Urban & 3 & 0.66 & 0.66 & 5.8 & 2.2 & 21,019 & 23,538 & 12,746 & 13,858 & 4.8 & 4.4 \\
\hline 13 & PPLT & Western & Urban & 3 & 0.66 & 0.66 & 5.8 & 2.2 & 13,490 & 14,824 & 4,602 & 4,737 & 1.4 & 0.5 \\
\hline 14 & PPLT & Western & Rural & 4 & 0.43 & 0.43 & 5.8 & 2.1 & 7,330 & 7,968 & 3,206 & 3,440 & 0.7 & 1.4 \\
\hline 15 & PPLT & Western & Urban & 3 & 0.77 & 0.77 & 5.3 & 3.7 & 25,592 & 24,743 & 3,321 & 3,184 & 3.9 & 5.1 \\
\hline 16 & PPLT & Western & Urban & 3 & 0.83 & 0.83 & 5.2 & 0.8 & 20,761 & 21,873 & 5,573 & 5,481 & 3.5 & 3.0 \\
\hline 17 & PPLT & Western & Urban & 4 & 0.60 & 0.60 & 5.0 & 1.9 & 5,528 & 5,409 & 3,377 & 3,146 & 1.6 & 2.0 \\
\hline 18 & PPLT & Western & Urban & 4 & 0.90 & 0.73 & 5.0 & 1.9 & 13,533 & 12,445 & 3,840 & 3,528 & 3.8 & 4.5 \\
\hline 19 & PPLT & Western & Urban & 3 & 0.74 & 0.74 & 5.0 & 1.9 & 7,798 & 7,002 & 6,917 & 6,811 & 1.8 & 4.0 \\
\hline 20 & PPLT & Western & Urban & 3 & 0.74 & 0.74 & 5.0 & 1.9 & 13,995 & 14,895 & 7,518 & 5,943 & 6.4 & 4.0 \\
\hline 21 & PPLT & Western & Urban & 4 & 0.47 & 0.47 & 5.3 & 3.7 & 27,680 & 27,409 & 8,550 & 8,815 & 17.4 & 11.2 \\
\hline 22 & PPLT & Western & Urban & 3 & 0.66 & 0.66 & 5.8 & 2.1 & 14,065 & 13,202 & 11,233 & 11,429 & 2.4 & 3.4 \\
\hline 23 & PPLT & Western & Rural & 4 & 0.55 & 0.55 & 5.1 & 1.8 & 8,400 & 8,402 & 4,313 & 4,339 & 2.2 & 2.5 \\
\hline 24 & PPLT & Western & Urban & 3 & 0.66 & 0.66 & 5.2 & 1.8 & 9,350 & 8,697 & 1,997 & 1,925 & 1.2 & 1.1 \\
\hline 25 & PPLT & Western & Urban & 4 & 0.62 & 0.62 & 5.8 & 2.1 & 8,469 & 7,779 & 3,314 & 3,685 & 2.9 & 2.4 \\
\hline 26 & PPLT & Western & Urban & 3 & 0.68 & 0.68 & 5.2 & 1.8 & 9,045 & 8,437 & 2,558 & 1,842 & 1.4 & 2.1 \\
\hline 27 & PPLT & Western & Urban & 3 & 0.66 & 0.66 & 5.2 & 1.8 & 6,549 & 5,180 & 2,412 & 2,243 & 0.8 & 0.0 \\
\hline 28 & PPLT & Northern & Urban & 3 & 0.68 & 0.68 & 5.2 & 3.8 & 10,257 & 10,849 & 9,254 & 10,244 & 5.0 & 3.5 \\
\hline 29 & PPLT & Northern & Rural & 4 & 0.67 & 0.67 & 4.6 & 4.3 & 15,092 & 16,034 & 6,268 & 7,519 & 7.2 & 9.0 \\
\hline 30 & $\mathrm{PO}$ & Eastern & Urban & 4 & 0.81 & 0.81 & 5.1 & 2.8 & 7,226 & 7,301 & 4,514 & 6,380 & 1.8 & 2.1 \\
\hline 31 & PO & Eastern & Urban & 3 & 0.89 & 0.89 & 5.7 & 2.3 & 37,199 & 34,740 & 1,942 & 1,958 & 1.8 & 1.3 \\
\hline 32 & $\mathrm{PO}$ & Eastern & Urban & 4 & 0.71 & 0.71 & 5.0 & 1.9 & 16,118 & 15,895 & 4,998 & 4,917 & 10.6 & 7.0 \\
\hline 33 & $\mathrm{PO}$ & Northern & Urban & 4 & 0.56 & 0.56 & 5.8 & 1.1 & 9,887 & 10,556 & 5,365 & 5,427 & 3.5 & 7.5 \\
\hline 34 & $\mathrm{PO}$ & Northern & Rural & 4 & 0.67 & 0.67 & 5.4 & 0.5 & 8,753 & 9,206 & 2,166 & 2,278 & 1.6 & 1.0 \\
\hline 35 & $\mathrm{PO}$ & Northern & Urban & 4 & 0.75 & 0.75 & 5.8 & 1.2 & 26,173 & 28,726 & 2,478 & 2,084 & 5.1 & 9.6 \\
\hline 36 & $\mathrm{PO}$ & Northern & Rural & 4 & 0.57 & 0.57 & 5.9 & 1.0 & 8,728 & 8,766 & 3,885 & 4,160 & 2.6 & 9.0 \\
\hline 37 & $\mathrm{PO}$ & Eastern & Urban & 3 & 0.79 & 0.79 & 5.9 & 2.0 & 31,933 & 33,011 & 7,151 & 7,450 & 2.5 & 9.0 \\
\hline & & & & & & & 5.3 & 2.0 & 13,343 & 13,489 & 4,641 & 4,773 & 3.4 & 3.6 \\
\hline
\end{tabular}




\subsubsection{Sites Installed Before 2016 vs All Sites}

Initially, two separate analyses were performed; one for only sites installed before 2016 and one for all sites, regardless of install year. Traditionally, data from the installation year of the treatment installation would be eliminated from CMF development. This would constrain the data analysis to include only sites installed before 2016. Since many sites underwent conversion in 2016, this had significant impacts on sample sizes. As an alternative, partial year data was also examined to assess whether it would provide a viable alternative in CMF development. Analysis of all sites, regardless of installation year was performed to develop final CMFs once it could be confirmed that using this dataset produced similar results to using a dataset comprised of only sites installed before 2016. This allowed for an extra site for each of the two conversion types, which would lead to more accurate results, using more data, especially for the protectedonly to PPLT-FYA cases, where data availability was already limited.

To complete this analysis, up to five full years and one partial year of before data, as well as up to five full years and one partial year of after data were used. In the calculations, partialyear data was accounted for by multiplying the number of predicted crashes in both the before and after periods by the number of months before or after divided by 12 . For example, for a site that was installed in March of 2016, the number of predicted crashes for 2016 in the before period was multiplied by $2 / 12$, and the number of predicted crashes for 2016 in the after period was multiplied by $9 / 12$. From this it should also be noted that the installation month data was not considered in the analysis, therefore in the above example only January and February were used in the before period and April through December in the after period for 2016. 


\subsubsection{Data Collection}

\subsubsection{Traffic Count Data}

A critical piece of data for each site selected for analysis was the annual traffic counts for each leg of the intersection, since the Virginia Safety Performance Functions (SPFs) used in the EB method calculations required them as a variable to predict the number of crashes at each location. This data was obtained from VDOT's Traffic Monitoring System (TMS) for each year from 2008 to 2016 for all sites. TMS is a database of VDOT's AADT information for each roadway link in Virginia. Some of the links that VDOT gathers count data for are not collected on a yearly-basis, therefore some sites have years missing. If a site did not have a traffic count on an approach for a given year, that count was calculated by linearly interpolating the counts from known years before and after that year. If the missing year of data did not have counts in years before and after it, the interpolation was not possible, thus the site was considered to have incomplete count data. This was important in determining which sites would be analyzed, as mentioned in the previous section of this chapter. It should also be noted that the counts that are in TMS are bi-directional counts, therefore these values were divided in half when recorded for this research, since only one direction of traffic would be entering the intersection for each leg. This was done so that if a roadway going through the intersection had two different AADTs (one on either side of the intersection) the average of the two links was used when combined.

Once all legs of each analysis intersection's AADT numbers were found, counts from opposite legs of the intersections were summed to represent one "road" going through the intersection. In the case of the three-leg intersections, the leg that did not have a leg opposite of it had its original single-directional count doubled, representing the original bi-directional AADT, as this is the convention for the Virginia SPFs. With this, each location then had two 
traffic counts representing roads through the intersection for every year, the higher of the two representing the "major" road AADT and the lower representing the "minor" road AADT. These values were used later in the analysis for the SPF since the minor and major road AADTs were needed.

\subsubsection{Crash Data}

To gather crash data for each intersection identified to be analyzed, VDOT's Roadway Network System (RNS) tool was used. With this database, crashes were identified for every site between 2008 and 2016 by a $250 \mathrm{ft}$ radius buffer around each intersection, which is the definition of intersection crashes used in the Virginia SPFs. From these crashes, the date, crash severity, and crash type were extracted to be used for analysis.

Crashes that were obtained from RNS were then reviewed to ensure they were associated with the intersection that was converted. Initially, the "route" information for each crash was compared to the intersection routes. If this field did not match either of the intersecting routes, the crash was discarded for the analysis. After this, each crash was mapped in VDOT's "Integrator" geographic information system (GIS). The crashes were then flagged for further analysis if they appeared as though they were associated with other intersections or if they appeared to be too far away from the intersection. More information for the flagged crashes was then found from RNS, such as crash diagrams and officer descriptions of the crashes. This information was used to determine if the crashes flagged were truly associated with the intersection, and if they were not, they were removed. This process was repeated for each intersection to be analyzed, and the remaining crashes were used for CMF calculations. 


\subsubsection{Site Characteristics}

Another vital set of information for the intersections were the site characteristics, so that base conditions could be checked. As a part of the EB method, if base conditions for sites are not met, CMFs other than the one that the analysis is developing must be calculated and applied to the number of predicted crashes. To check if the conditions were met or if CMFs had to be determined, the following site characteristics were found for each site in Table 4.

Table 4 - Site Characteristics

\begin{tabular}{c|c|c} 
Urban/Rural & Characteristic & Values \\
\hline \hline \multirow{5}{*}{ Both } & Location type & Urban, Rural \\
\cline { 2 - 3 } & \# of legs & 3,4 \\
\cline { 2 - 3 } & $\begin{array}{c}\text { \# of legs with left-turn } \\
\text { lanes }\end{array}$ & $0,1,2,3,4$ \\
\cline { 2 - 3 } & $\begin{array}{c}\text { \# of legs with right } \\
\text { turn lanes }\end{array}$ & $0,1,2,3,4$ \\
\hline \hline \multirow{6}{*}{ Urban } & $\begin{array}{c}\text { \# of legs with left-turn } \\
\text { phasing }\end{array}$ & $0,1,2,3,4$ \\
\cline { 2 - 3 } & $\begin{array}{c}\text { \# of legs with right } \\
\text { turn on red prohibited }\end{array}$ & $0,1,2,3,4$ \\
\cline { 2 - 3 } & $\begin{array}{c}\text { Left-turn Phasing (for } \\
\text { each approach) }\end{array}$ & $\begin{array}{c}\text { Permissive, } \\
\text { Protected, PPLT }\end{array}$
\end{tabular}

With these characteristics, CMF values were determined for each location using values presented in the HSM (26). To find these site characteristics, Google Maps and Google Street View were used to view imagery of the locations. It should be noted that whether a site was rural or urban was based on VDOT's functional classification of the roads at the intersection. Additionally, no rural-specific base conditions were evaluated since only intersection skew is reported in the HSM, which is not a factor for signalized intersections. Although no rural- 
specific base conditions were included, the distinction between base CMFs for rural and urban intersections with respect to the number of approaches with left-turn lanes and the number of approaches with right turn lanes were accounted. Base CMFs for left-turn phasing were only accounted for in the PPLT to PPLT-FYA conversion cases, as the final CMFs that were calculated for the protected-only to PPLT-FYA account for the phasing change. It should also be noted that one urban intersection's geometry changed concurrently with the signal conversion, which added two left-turn lanes, one in each direction on the mainline roadway. This was accounted for in the before and after CMFs for this intersection. No other intersections in the analysis had changes in base conditions between the before and after periods.

\subsubsection{CMF Calculations}

Separate analyses were performed for the three- and four-leg intersections for the conversion from PPLT to PPLT-FYA, and analyses for the conversion from protected-only to PPLT-FYA included both three- and four-leg intersections together as there were only two threeleg intersections. In this case, there were 16 three-leg sites and 13 four-leg sites for the PPLT to PPLT-FYA conversion, and 8 total sites for the protected-only to PPLT-FYA conversion. As stated before, using partial-year data provided for a larger set of data to be analyzed over using only full-year data, which would increase the accuracy of the results by representing different locations.

\subsubsection{EB Method}

The predicted number of crashes summed across all years in the before period determined using the Virginia SPFs and then multiplied by the corresponding CMFs determined for that 
intersection to determine the predicted crashes for the before period. This process is repeated for the after period using the after-period CMFs to calculate the number of predicted crashes in the after years. The number of expected crashes is determined by applying a weighting factor, as calculated using the appropriate overdispersion factor, $\mathrm{k}$, for the intersection, to the observed and predicted number of crashes. The weighting factor, w, determines what percentage of predicted crashes should be used and what remaining percentage $(1-$ w) of observed crashes should be used in deciding the expected crashes in the before period. The equation for calculation of the weighting factor is shown in Equation 2, and the equation for calculation of the number of expected crashes in the before period is shown in Equation 3.

$$
w=\frac{1}{1+k\left(\sum_{\text {all before years }} N_{\text {predicted }}\right)}
$$

where

$$
\begin{aligned}
& w=\quad \text { weighting factor (decimal), } \\
& k=\quad \text { overdispersion factor from SPF (decimal), and } \\
& \sum_{\text {all before years }} N_{\text {predicted }}=\begin{array}{l}
\text { sum of SPF-predicted crashes for all study years, } \\
\text { for the before period. }
\end{array}
\end{aligned}
$$

$$
N_{\text {expected }}=w N_{\text {predicted }}+(1-w) N_{\text {observed }}
$$

where

$$
\begin{aligned}
& N_{\text {expected }}=\text { number of expected crashes in the before period, and } \\
& N_{\text {observed }}=\text { number of field-observed crashes in the before period. }
\end{aligned}
$$

The number of expected crashes, along with the ratio of the predicted number of crashes in the after period to the predicted number of crashes in the before period, is then used to calculate the number of expected crashes in the after period. From there, this value is compared 
to the observed number of crashes at each site to find an Odds Ratio (OR). The overall OR is then calculated using the summation of all observed crashes at all locations and dividing that by the summation of all predicted crashes at all locations. Finally, a correction is applied to this value to formulate the final $\mathrm{CMF}$ value for the analysis, the equation for which is found below in Equation 4.

$$
O R=\frac{O R^{\prime}}{1+\frac{\sum_{\text {All sites }} r_{i}^{2} N_{\text {expected }, B}\left(1-w_{i}\right)}{\left(\sum_{\text {All Sites }} N_{\text {expected }, A}\right)^{2}}}
$$

where

$$
\begin{aligned}
& \mathrm{OR}=\text { unbiased, final odds ratio }(\mathrm{CMF}), \\
& \mathrm{OR}^{\prime}=\text { unadjusted odds ratio, and } \\
& r_{i}=\text { adjustment factor (ratio of predicted crashes before to after). }
\end{aligned}
$$

\subsubsection{Angle Crashes}

In addition to all crash type CMF calculations, angle crashes were analyzed, since these should have been the crash type most affected by the conversion from PPLT or protected-only PPLT-FYA. To correct for only angle crash types, the number of observed crashes for each year only counted angle crashes, and the number of predicted crashes for both the before and after periods were multiplied by the percentage of angle crashes to total crashes before the conversion, as advocated by the HSM (26). The percentages of angle crashes used in the calculations are listed below in Table 5. 
Table 5 - Percentage of Angle Crashes in the Before Period

\begin{tabular}{|c|c|c|c|}
\hline \multirow[b]{2}{*}{$\begin{array}{c}\text { Conversion } \\
\text { Type }\end{array}$} & \multirow[b]{2}{*}{$\begin{array}{l}\text { Intersection } \\
\text { Geometry }\end{array}$} & \multicolumn{2}{|c|}{$\%$ Angle Crashes } \\
\hline & & $\begin{array}{l}\text { All Crash } \\
\text { Severities }\end{array}$ & $\begin{array}{l}\text { Fatal \& Injury } \\
\text { Crash Severities }\end{array}$ \\
\hline \multirow{3}{*}{$\begin{array}{c}\text { PPLT } \\
\text { to PPLT-FYA }\end{array}$} & All & $49 \%$ & $56 \%$ \\
\hline & 3-leg & $43 \%$ & $50 \%$ \\
\hline & 4-leg & $53 \%$ & $61 \%$ \\
\hline $\begin{array}{l}\text { Protected-Only } \\
\text { to PPLT-FYA }\end{array}$ & All & $29 \%$ & $28 \%$ \\
\hline
\end{tabular}

\subsection{RESULTS}

\subsubsection{Sites Installed Before 2016 vs All Sites}

Using partial-year data for the "all sites" calculation method increased the amount of crash data that could be used, which was especially important since a majority of the analysis sites were installed within two years of the analysis, making after data limited. A comparison of the two CMFs calculated are shown below in Table 6 and Table 7, with bold face and italicized CMFs being statistically significant at the $95 \%$ confidence level and italicized CMFs being statistically significant at the $90 \%$ confidence level. For the all sites cases, two additional sites were able to be analyzed, one from each conversion type. Due to the increased number of sites and the increased number of months that could be analyzed, CMFs for the all sites calculations had standard error values lower than the sites that were installed before 2016, which made the CMFs more precise. Additionally, the values produced by both datasets were similar, as their $95 \%$ confidence intervals overlap in all cases, with the confidence interval being defined as the CMF plus or minus the product of the standard error and 1.96. Since they had lower standard errors, the "all sites" calculation were used for CMF development because of the additional data availability. 
Table 6 - CMF Comparison for All Crash Severities

\begin{tabular}{|c|c|c|c|c|c|c|}
\hline \multirow{3}{*}{ Conversion } & \multirow{3}{*}{ Crash Type } & \multicolumn{3}{|c|}{ All Severities } & \multirow{2}{*}{\multicolumn{2}{|c|}{ Pre-2016 }} \\
\hline & & & & Sites & & \\
\hline & & $\pi$ Legs & CMF & Std. Err. & CMF & Std. Err. \\
\hline \multirow{6}{*}{$\begin{array}{l}\text { PPLT to } \\
\text { PPLT-FYA }\end{array}$} & \multirow{3}{*}{ all } & all & 0.95 & 0.075 & 1.00 & 0.090 \\
\hline & & 3 & 0.96 & 0.123 & 1.03 & 0.148 \\
\hline & & 4 & 0.94 & 0.096 & 0.98 & 0.113 \\
\hline & \multirow{3}{*}{ angle } & all & 0.71 & 0.090 & 0.76 & 0.107 \\
\hline & & 3 & 0.62 & 0.130 & 0.65 & 0.152 \\
\hline & & 4 & 0.77 & 0.121 & 0.83 & 0.147 \\
\hline \multirow{2}{*}{$\begin{array}{c}\text { Protected- } \\
\text { Only to } \\
\text { PPLT-FYA }\end{array}$} & all & all & 1.47 & 0.206 & 1.79 & 0.274 \\
\hline & angle & all & 2.56 & 0.525 & 3.14 & 0.700 \\
\hline
\end{tabular}

Table 7 - CMF Comparison for Fatal and Injury Crash Severities

\begin{tabular}{|c|c|c|c|c|c|c|}
\hline \multirow{3}{*}{ Conversion } & \multicolumn{4}{|c|}{ FI Severities } & & \\
\hline & \multirow{2}{*}{ Crash Type } & \multirow{2}{*}{ \# Legs } & \multicolumn{2}{|c|}{ All Sites } & \multicolumn{2}{|c|}{ Pre-2016 } \\
\hline & & & CMF & Std. Err. & CMF & Std. Err. \\
\hline \multirow{6}{*}{$\begin{array}{c}\text { PPLT to } \\
\text { PPLT-FYA }\end{array}$} & \multirow{3}{*}{ all } & all & 0.99 & 0.131 & 1.07 & 0.159 \\
\hline & & 3 & 0.70 & 0.152 & 0.71 & 0.183 \\
\hline & & 4 & 1.26 & 0.211 & 1.37 & 0.255 \\
\hline & \multirow{3}{*}{ angle } & all & 0.87 & 0.159 & 0.92 & 0.191 \\
\hline & & 3 & 0.63 & 0.194 & 0.63 & 0.220 \\
\hline & & 4 & 1.05 & 0.243 & 1.20 & 0.311 \\
\hline \multirow{2}{*}{$\begin{array}{c}\text { Protected- } \\
\text { Only to } \\
\text { PPLT-FYA }\end{array}$} & all & all & 1.57 & 0.328 & 2.13 & 0.475 \\
\hline & angle & all & 3.30 & 0.955 & 4.01 & 1.227 \\
\hline
\end{tabular}

\subsubsection{CMFs}

CMFs and their corresponding standard errors and significance levels were computed, the values of which can be found below in Table 8 and Table 9, with bold face and italicized CMFs 
being statistically significant at the $95 \%$ confidence level and italicized CMFs being statistically significant at the $90 \%$ confidence level. The angle crash CMFs for the PPLT to PPLT-FYA conversion type and for all severities were statistically significant to the $90 \%$ confidence level or better, with the all and three-leg CMFs statistically significant to the $95 \%$ confidence level. Also, the three-leg CMFs for the PPLT to PPLT-FYA conversion for all and angle crash types for fatal and injury crash severities were significant to the $90 \%$ confidence level.

For the protected-only to PPLT-FYA conversion, the all crash types CMF was statistically significant to the $90 \%$ confidence level for fatal and injury severities, and the angle crash CMFs for all and fatal injury severities, as well as the all crash types for all injury type CMF were statistically significant to the $95 \%$ confidence level.

Table 8 - CMF Results for All Crash Severities

\begin{tabular}{|c|c|c|c|c|}
\hline Conversion & Crash Type & \# Legs & CMF & Std. Err. \\
\hline \multirow{6}{*}{$\begin{array}{l}\text { PPLT to } \\
\text { PPLT-FYA }\end{array}$} & \multirow{3}{*}{ all } & $\overline{\text { all }}$ & 0.95 & 0.075 \\
\hline & & 3 & 0.96 & 0.123 \\
\hline & & 4 & 0.94 & 0.096 \\
\hline & \multirow{3}{*}{ angle } & all & 0.71 & 0.090 \\
\hline & & 3 & 0.62 & 0.130 \\
\hline & & 4 & 0.77 & 0.121 \\
\hline \multirow{2}{*}{$\begin{array}{c}\text { Protected- } \\
\text { Only to } \\
\text { PPLT-FYA }\end{array}$} & all & all & 1.47 & 0.206 \\
\hline & angle & all & 2.56 & 0.525 \\
\hline
\end{tabular}


Table 9 - CMF Results for Fatal and Injury Crash Severities

\begin{tabular}{c|c|c|c|c} 
Conversion & Crash Type & \# Legs & CMF & Std. Err. \\
\hline & & all & 0.99 & 0.131 \\
& \multirow{4}{*}{ all } & 3 & 0.70 & 0.152 \\
PPLT to & & 4 & 1.26 & 0.211 \\
\cline { 3 - 5 } PPLT-FYA & & all & 0.87 & 0.159 \\
& \multirow{2}{*}{ angle } & 3 & 0.63 & 0.194 \\
& & 4 & 1.05 & 0.243 \\
\hline \hline Protected- & \multirow{2}{*}{ all } & all & 1.57 & 0.328 \\
Only to & angle & all & $\mathbf{3 . 3 0}$ & $\mathbf{0 . 9 5 5}$ \\
\cline { 3 - 5 } PPLT-FYA & ang & & &
\end{tabular}

\subsubsection{PPLT to PPLT-FYA CMFs}

As found in Table 8, the PPLT to PPLT-FYA conversion for angle crashes created a 29\%, $38 \%$, and $23 \%$ reduction in crashes for all, three-, and four-leg intersection types, respectively, the first two of which were statistically significant at the $95 \%$ level, and the third at the $90 \%$ level. In addition to this, fatal and injury crash severities for all and angle crash types at threeleg intersections created a $30 \%$ and $37 \%$ crash reduction, both of which being significant at the 90\% level. These show the general trend of a decrease in crashes with this conversion type.

\subsubsection{Protected-Only to PPLT-FYA CMFs}

As found in Table 8 and Table 9, the protected-only to PPLT-FYA conversion resulted in an increase in crashes for the all and angle crash types as well as all and fatal and injury severities. The increase in crashes can be quantified as $47 \%$ for all crash types and severities, $57 \%$ for all crash types and fatal and injury severities, $156 \%$ for angle crash types and all severities, and finally $230 \%$ for angle crash types and fatal and injury severities. The second number listed is significant to the $90 \%$ confidence level and the others are significant to the $95 \%$ 
confidence level. From this, all crash types increase for this conversion type, but angle crashes increase by much more significant margins.

\subsubsection{Summary of Findings}

Using a before and after study with the Empirical Bayes (EB) method, CMFs were calculated for the conversion from PPLT to PPLT-FYA, the values of which can be found below.

- Angle crashes for all intersection types and all severities: 0.71, significant at the $95 \%$ confidence level,

- Angle crashes for 3-leg intersections and all severities: 0.62, significant at the 95\% confidence level,

- Angle crashes for 4-leg intersections and all severities: 0.77, significant at the 90\% confidence level,

- Angle crashes for 3-leg intersections and fata and injury severities: 0.63 , significant at the $90 \%$ confidence level, and

- All crash types for 3-leg intersections and fatal and injury severities: 0.70, significant at the $90 \%$ confidence level.

Additionally, CMFs were calculated for the conversion from protected-only to PPLTFYA, shown below.

- All crash types and all severities: 1.47, significant at the 95\% confidence level,

- Angle crash types and all severities: 2.56, significant at the 95\% confidence level,

- All crash types and fatal and injury severities: 1.57, significant at the $90 \%$ confidence level, and 
- Angle crash types and fatal and injury severities: 3.30, significant at the 95\% confidence level.

All of the CMFs had relatively large confidence intervals, which shows the variability of the effect of the conversion between different sites

The reduction in angle crashes represented by the CMFs for green ball to FYA conversions supports the findings of previous studies that drivers understand FYA better than green ball. Additionally, with the CMFs developed, practitioners and researchers are now able to quantify the safety effect of converting PPLT traffic signals from green ball to FYA permissive left-turn indication for angle crashes at three- and four-leg intersections for all crash severities, as well as at three-leg intersections for fatal and injury crash severities.

For conversions from protected-only phasing to PPLT-FYA, increases in crashes represented by the CMFs also support the theory that less-restrictive left-turn phasing increases crashes, although the important contribution was the quantification of this increase. The target crash type, angle crashes, showed a much larger increase than for all crash types, which is to be expected. Additionally, the CMF for fatal and injury crash severities for angle crash types was higher than the angle crash CMF for all crash severities, which is likely explained by the thought that angle crashes are typically more severe than other crash types.

In reviewing the results presented above, the reliable CMFs that were calculated are the angle crash CMFs for all crash severities and all and angle crash type for three-leg intersection CMFs for fatal and injury crash severities for the green ball to FYA conversion, and all CMFs developed for the protected-only to PPLT-FYA conversion. Although they are statistically significant, the angle CMFs for the protected-only to PPLT-FYA conversion had large confidence intervals, most likely due to only having eight intersections to analyze. 
Comparing the results of this study to the CMFs developed by the previously mentioned papers by Srinivasan, et al. (13) and Simpson and Troy (2), the CMF value for this study for angle crashes and all severities for the green ball to FYA conversion was 0.71 , which is close to the 0.81 and 0.84 values found by the other studies, respectively, considering the confidence interval overlap. The CMFs developed by this study corresponding to the conversion from protected-only to PPLT with FYA for all and angle crash types for all severities were also close to those determined by Srinivasan, et al. of 1.34 and 2.24, respectively. Although the values found by this paper were not exactly what were found in the literature, the confidence intervals overlapped, meaning that with more data, lower standard errors could be produced. 


\section{CHAPTER 4: LEFT-TURN CAPACITY AND CONFLICT MODELS}

After determining the safety effects of left-turn phasing mode and displays, models were created to predict left-turn capacities and the number of crossing conflicts based on simulation data for permissive-only and PPLT phasing modes. Since the results in Chapter 3 focused on static phasing mode choice, models developed in this chapter were critical in the evaluation timeof-day operations of signals, as they take into consideration intersection parameters that change throughout the day, affecting operations and safety dynamically. In modeling combinations of traffic signal timing parameters, traffic volumes, and intersection characteristics, the effects of these variables could be analyzed and used in time-of-day phasing decisions.

For the operational models, left-turn capacities were simulated in the software package PTV VISSIM 8 based on several input variables. Trajectory files were also obtained from the simulations for processing in the FHWA's Surrogate Safety Assessment Model (SSAM) software to determine the number of crossing conflicts from each simulation run. The conflict models were developed from these outputs and the input variables used in the simulations. The models created in this part of the research will allow engineers to determine the operational and safety effects of different signal timing, traffic, and geometric variables.

\subsection{METHODOLOGY}

\subsubsection{VISSIM Simulations}

To evaluate the impacts of signal parameters, traffic characteristics, and geometric conditions on the operations and safety of permissive-only and PPLT phasing control modes, simulations were run in PTV's VISSIM 8. Simulation modeling was chosen so that variables could be controlled, and hundreds of combinations of input variables could be tested with 
relative ease. VISSIM, a microsimulation program that models individual vehicles on the roadway network, was used as the simulation software for the research since it had the ability to be run via a script to automate scenario creation so that the inputs of hundreds of scenarios did not have to be entered manually. Additionally, VISSIM supported the tracking of individual vehicles' paths through trajectory (TRJ) files that would be later analyzed for conflicts in SSAM.

\subsubsection{Experimental Design}

The first step in creating simulation models for the research was to select the parameters that would be used in the simulations. It was also important to decide which parameters would be constant in each model, and which would vary from model to model. Ultimately, eight variables were chosen to be simulated, shown below in Table 10. Each variable is discussed separately in subsequent sections.

Table 10 - Simulation Parameters

\begin{tabular}{c|c} 
Parameter & Values/Range \\
\hline Area Type & Rural, Urban \\
\hline \# of Opposing Lanes & $1,2,3$ lanes \\
\hline Left-Turn Mode & Permissive-Only, PPLT-Lead \\
\hline Protected Green Ratio (PPLT) & $0.10,0.15,0.20,0.25$ \\
\hline Cycle Length & $80-240 \mathrm{~s}$ \\
\hline Green Ratio & $0.3-0.8$ \\
\hline Opposing Volume & $200-1,200 \mathrm{veh} / \mathrm{hr} / \mathrm{ln}$ \\
\hline Average Opposing Speed & $35-55 \mathrm{mph}$
\end{tabular}

\subsection{Area Type}

Urban and Rural area types were chosen to be modeled to distinguish between results with different saturation flow rates. As defined by the Highway Capacity Manual (HCM) (27), 
the defining traffic characteristic between the two areas is the ideal saturation flow rate. For urban areas, a value of 1,900 vehicles per hour per lane (veh/hr/ln) is assumed, and for rural areas, a value of 1,750 is assumed.

\subsection{Number of Opposing Lanes}

As the number of opposing through lanes that the left-turning vehicles must cross has been proven to be significant in the efficient operations and safety of left-turns in past studies (1) (4) (11) (14) (16) (17) (18) (19) (20) (21) (22) (23) (24), this was decided to be included as a variable in the simulations. One, two, and three opposing lanes were used as the levels of this variable, as less-restrictive left-turn phasing modes such as permissive-only and PPLT are extremely uncommon at intersections with more than three opposing through lanes in Virginia.

\subsection{Left-Turn Phasing Mode}

With the purpose of this research being to analyze the impacts of different left-turn phasing control modes on the safety and operations of left-turns, permissive-only and PPLT control modes were selected as the two left-turn phasing modes for simulation. The third type of phasing control mode, protected-only, was not simulated, as left-turn capacities could easily be determined for this mode analytically, and the safety of this mode is generally understood. Furthermore, since VISSIM simulates fundamentally safe driving behavior, few if any left turn conflicts should be generated from protected only phasing. If protected-only phasing was modeled, the number of unique scenarios would increase by $50 \%$, making running and processing the simulations take far longer. Since the behavior of protected-only phasing is already well-understood, simulating the phasing mode was omitted from this analysis. It should 
also be noted that for all PPLT scenarios, the protected portion of the phase was always the leading portion.

\subsection{Protected Green Ratio}

Since PPLT phasing can have many different protected-portion green times, a ratio of protected green time to the cycle length for PPLT was used as another simulation parameter. It should also be noted that the numerator of the ratio includes yellow and all-red times; i.e. a protected green ratio of 0.1 and cycle length of 90 seconds $(\mathrm{s})$ would have $0.1 * 90 \mathrm{~s}=9 \mathrm{~s}$ of combined green, yellow, and red times. Four levels of the protected green ratio were used in the simulation models: $0.10,0.15,0.20$, and 0.25 , corresponding to $10 \%, 15 \%, 20 \%$, and $25 \%$ of the signal cycle given to the protected portion of the subject approach left-turn, respectively. Ultimately, this was reflected in the left-turn mode parameter as one permissive-only left-turn mode $(0 \%$ protected) and the four levels of PPLT. These levels were selected in consultation with a panel of VDOT traffic engineers as being representative of common PPLT protected portions in Virginia.

\subsection{Cycle Length}

The length of the signal cycle was also accounted for as a variable in the simulation scenarios. A range of cycle lengths from 80 s to 240 s was used to capture most common possible values used in Virginia for traffic signals. This simulation parameter, along with the subsequent three parameters, was used as a continuous variable in determining the simulation scenarios, thus discrete values of this variable were not used. 


\subsection{Subject Street Green Ratio}

Similar to the protected green ratio, the subject street green ratio, or simply green ratio, was calculated as the ratio of the green time given to the subject "street" (the combination of two opposite approaches at the intersection) to the cycle length. This number determined the proportion of the cycle length given to the subject street, and the remaining time given to the cross-street. For example, if the green ratio was 0.6 , the subject street would get $60 \%$ of the cycle and the cross-street would get $40 \%$ of the cycle. It should also be noted that the simulations were set up in a manner that only one approach was identified as the subject left-turn approach and only one approach was identified as the opposing through approach, both of which are on the same street, corresponding to the subject street. The value of green ratios used in the simulation ranged from 0.3 to 0.8 as this was deemed to be a realistic, based on guidance from a panel of VDOT traffic engineers. The green ratio was also a continuous simulation parameter.

\subsection{Opposing Volume}

Opposing through volume, in units of vehicles per hour per lane, is widely accepted as one of the most influential factors on left-turn capacity and safety for permissive left-turns, as it determines the number gaps available to execute left-turns. As such, the opposing volume was included in the simulations as a continuous variable from $200 \mathrm{veh} / \mathrm{hr} / \mathrm{ln}$ to $1,200 \mathrm{veh} / \mathrm{hr} / \mathrm{ln}$. The range of opposing volumes was considered to be appropriate, as opposing volumes less than 200 $\mathrm{veh} / \mathrm{hr} / \mathrm{ln}$ would provide enough safe gaps for permissive left-turning vehicles. Volumes above 1,200 veh/hr/ln would likely have few safe gaps, thus protected-only phasing would be

recommended over this value. This parameter was represented in the VISSIM models by changing the "Volume" field in the vehicle inputs attribute for the opposing through lanes link. 
Since this value is inputted as vehicles per hour (veh/hr), the desired volumes in veh/hr/ln were multiplied by the number of lanes for the scenario before entering the value.

\subsection{Average Speed for Opposing Vehicles}

The final continuous variable, the speed for opposing through vehicles, ranged from 35 $\mathrm{mph}$ to $55 \mathrm{mph}$. This parameter was accounted for in the simulations by using it to determine the desired speed distributions. This was done by setting the average speed of through vehicles equal to the speed limit, the standard deviation of speeds to $5 \mathrm{mph}$, and the minimum and maximum values being plus or minus $10 \mathrm{mph}$ respectively, assuming a normal distribution. The speed limit was used as a simulation variable as it was determined to affect permissive left-turn modes by other studies, mentioned in (11) (14) (16) (17) (19) (20) (21) (22) (23) (24).

\subsection{Constants}

One constant parameter, percentage of heavy-goods vehicles, was set to 0.001 in VISSIM, to reflect roughly $0 \%$ trucks, as VISSIM does not allow the value to be exactly zero. This parameter did not vary in the simulations since adjustment factors for different percentages of trucks can be applied after the capacities for an intersection are determined, as defined in the HCM.

Another factor that was held constant throughout the simulations was the percent grade of the approaching roadways. This parameter was not studied as a part of the simulation modeling as the effect of roadway grade on operations and safety of an intersection is generally understood. Therefore, this parameter can be accounted for by engineers after they receive predictions from the models developed by this study. 
Finally, the yellow and all-red clearance times were held constant throughout the scenarios at values of four seconds and one second, respectively. These durations corresponded to the amount of time the yellow indication on the phase that was ending was displayed and the amount of time the red indication for all approaches was shown to clear the intersection for the next phase. In the case of PPLT switching from the protected to the permitted portion of the phase, the all-red clearance time was considered the PPLT clearance time, as the red indication was only shown on the left-turn approach rather than to all approaches. This value also was one second, thus when the protected portion of the PPLT phase ended, four seconds of yellow and one second of red was shown to the left-turn approach before beginning the permitted portion of the phase.

\subsubsection{Base Model Development}

A base model was first created so that the model could be calibrated to ensure accurate results. The geometry of the intersection of U.S. Route 29 and Timberwood Boulevard in Albemarle County, Virginia was replicated for the VISSIM base model, and the lanes not used in calibration were "blocked" in the model so that traffic was only produced on one left-turning lane and the two opposing through lanes that were closest to the median. The reason this intersection was replicated was that it provided the ability to expand the geometry options of the simulation to any desired combination of up to two left-turn lanes and up to three opposing through lanes.

Stop bar locations, conflict areas, and a data collector on the lane that received the leftturning vehicles were added. The conflict areas were a vital piece to the models because they were the only way to define the behavior of a permissive left-turn phase in VISSIM. A conflict 
area in the software is defined as a location where two links overlap, creating an area where conflicts may occur. These areas are used to establish which direction of travel has the right of way. Since VISSIM does not allow the signal phases to utilize both protected and permissive phases, the conflict area used established that the opposing through traffic always had the right of way. This worked since it applied all times green was displayed for permissive left-turns, and for protected left-turns there was no opposing through traffic traversing the intersection, thus the conflict zone was ignored by the turning vehicles in these instances.

The stop bars in the model were located at logical spots for the intersection, based on the intersection referenced in creating the model. Additionally, the base simulation model was configured such that if left-turning vehicles were waiting for opposing vehicles to pass, two leftturning vehicles were past the stop bar. This was done so that when the signal indication turned red, these vehicles would clear the intersection, executing the left-turn. The two vehicles that "sneak" through the intersection at the end of the permissive indication are considered left-turn sneakers. As stated in the HCM, an average value of two sneakers per cycle in permissive indications should be assumed. This was achieved by setting the "AddStopDist" parameter in the conflict area's attributes to a value that would produce two sneakers per cycle. This parameter depends on the geometry of the intersection and the location of the conflict area; therefore, several values were tested to determine if they produced the desired number of sneakers. A final value of 40 was used for the parameter.

A data collector was positioned on the receiving end of the turn, just past the final opposing lane that the turning traffic had to cross. This data collector acted similar to a roadway tube used on an actual roadway to collect traffic counts as it counted each vehicle that passed over it for the duration of the simulation. 
To determine the capacity of the simulation runs, the left-turn link volumes were set to 2,000 veh/hr in the "Vehicle Inputs" asset for the road link to ensure that the lane was oversaturated. By doing this, the counts produced by the data collector for the link were considered to be left-turn capacities, as they were the maximum number of vehicles that could be served by the particular simulation run. Additionally, all simulation runs lasted for 75 minutes, with 60 minutes of data collection. Data collection did not occur during the first 15 minutes to ensure that the traffic volumes reached equilibrium values. In doing this, accurate simulation runs were produced, and the capacity numbers from the simulations were in vehicles per hour units.

Finally, a VAP file for determining signal timing was created. Since the signal timing variables used in the simulations were to be tested to determine their impact on left-turn capacity and conflicts, a fixed-time signal plan was used in the VAP file. In running each scenario in the simulations, the VAP file was automatically updated to reflect the combination of phasing mode, protected green ratio, cycle length, and subject street green ratio variables being simulated through a script developed by another researcher. These parameters determined the display sequence and green times given to each intersection approach. In each of the simulation runs for PPLT phasing, the subject street's left-turn approaches started first with the protected portion of their phase. Once this portion's time elapsed, the through movements' green time would start, with the left-turn approaches having permissive time. Once that time elapsed, all of one side street's approaches would go, followed by the all of other side street's approaches, as the side street was set up as split phasing. For the permissive-only runs, a similar sequence would occur, with the exception that the subject street's left-turn approaches were not given any protected green time. Therefore, the subject street's green time consisted of only the two through approaches, with permissive time for the left-turns occurring at the same time. 


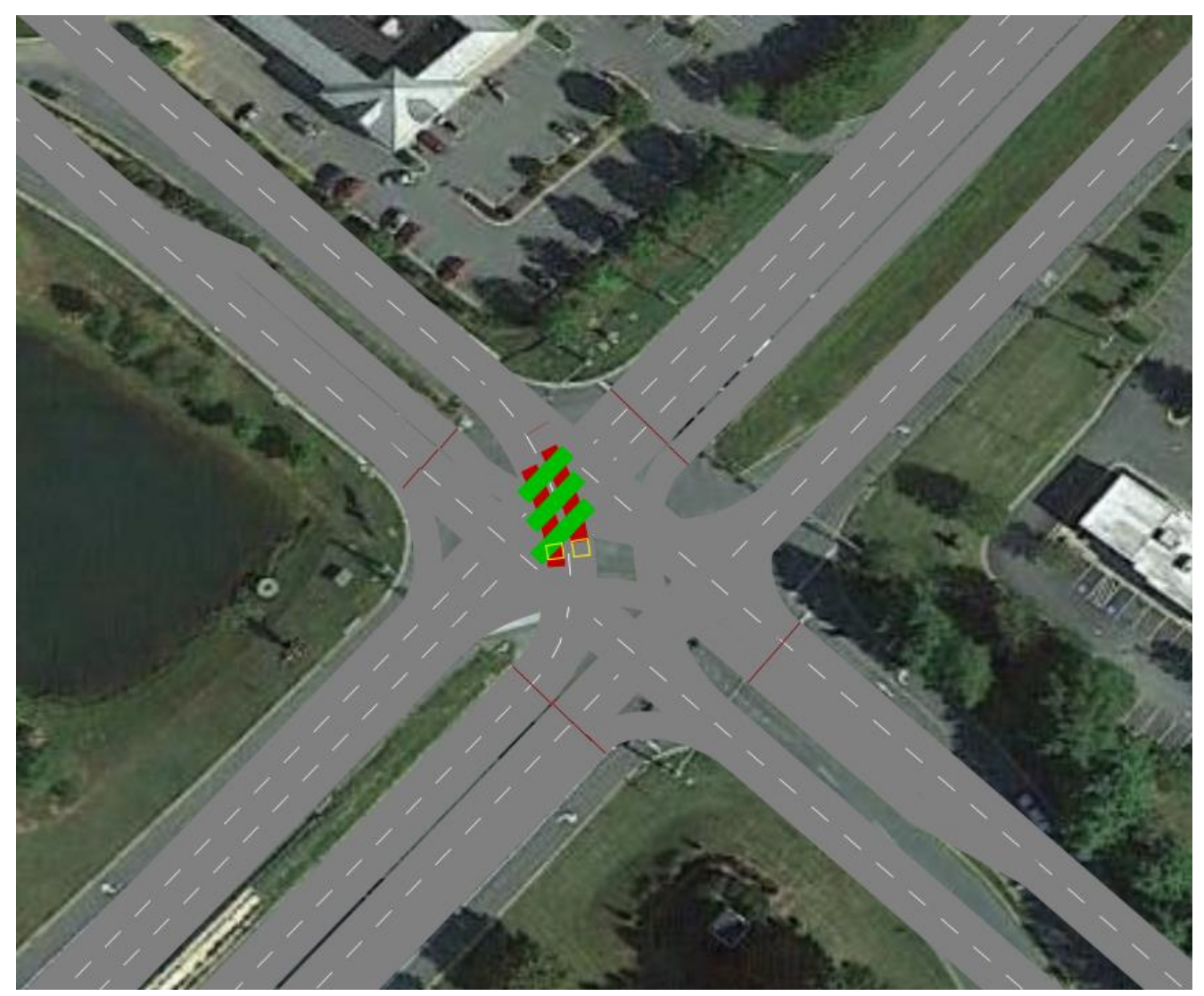

Figure 11 - VISSIM Base Model

In Figure 11 above, the VISSIM Base Model is shown, with the subject analysis street going from the bottom left of the image to the top right of the image. The subject left-turn is represented by the lanes starting from the bottom left corner of the image and traversing across the intersection to the top left. The conflict areas, shown as the red and green polygons, are positioned such that they cross the left-turn and opposing through lanes. The red in the conflict zones represents the left-turn lanes, as they must wait for the opposing lanes during the permissive portion of the signal phase, and the green represents the opposing through lanes, which always have right of way when given a green indication. The data collector can be seen as a small line crossing the outer left-turn lane, just past the conflict areas. Finally, stop bars are shown as red lines that cross each intersection approach. 


\subsubsection{Model Calibration}

In creating a model that accurately represents theoretical conditions, two forms of calibration were completed: one for ensuring the saturation flow rates for the urban and rural cases were correct, and one for ensuring the model produced accurate capacity estimates. For the first calibration, the Wiedemann 74 car following models used in VISSIM were calibrated to produce the appropriate saturation flow rates. Five simulations were run for thirteen different combinations of the multiplicative and additive part of desired safety distance factors to determine headways for vehicles in the left-turn and opposing through directions separately. Headway values for the fifth through tenth vehicles were averaged across the five runs and for $2^{\text {nd }}$ through $29^{\text {th }}$ signal cycles, producing an average of 840 values. Then, 3600 seconds per hour was then divided by these values, in seconds per vehicle per lane, to determine saturation flow rates in vehicles per hour per lane. Results from these calculations are shown below in Table 11, with the bold face rows representing the successful combination of parameters to produce urban and rural saturation flow rates, respectively. It should also be noted that the ideal saturation flow rates that were desired were $1,900,1,805,1,750$, and 1,663 for urban through lanes, urban leftturn lanes, rural through lanes, and rural left-turn lanes, respectively. Lower values for saturation flow rates for the left-turns were desired, as left-turn saturation flow rates are assumed to be $95 \%$ of the through lane saturation flow rates. In following the aforementioned procedure, it was found that a value of 4.00 for the multiplicative part of desired safety distance and a value of 3.25 for the additive part of desired safety distance were optimal for urban saturation flow rates, and values of 4.75 and 3.75 respectively were optimal for rural saturation flow rates. 
Table 11 - Saturation Flow Rate Calibration

\begin{tabular}{cc|cc}
$\begin{array}{c}\text { Multiplicative } \\
\text { Part of Safety }\end{array}$ & $\begin{array}{c}\text { Additive Part } \\
\text { of Safety }\end{array}$ & \multicolumn{2}{|c}{ Saturation Flow } \\
Left-Turn & Through \\
\hline 3 & 2 & 2103 & 2199 \\
3.25 & 2.25 & 2041 & 2163 \\
3.5 & 2.25 & 2025 & 2122 \\
3.75 & 2.25 & 2017 & 2115 \\
3.75 & 2.5 & 1985 & 2056 \\
3.75 & 2.75 & 1940 & 2030 \\
3.75 & 3 & 1907 & 1974 \\
4 & 3 & 1879 & 1957 \\
4 & 3.25 & 1840 & 1917 \\
4.5 & 3.5 & 1777 & 1842 \\
4.75 & 3.5 & 1754 & 1819 \\
$\mathbf{4 . 7 5}$ & 3.75 & 1720 & 1783 \\
5 & 3.75 & 1706 & 1770
\end{tabular}

Once a base model was formed, calibration had to be performed to ensure the results reflected theoretical values calculated by HCM procedures. To achieve this, 56 scenarios were developed and run using the base model to compare the simulated capacities to HCM-calculated capacities. In each scenario, two opposing lanes, a $45 \mathrm{mph}$ speed limit for opposing vehicles, and permissive-only left-turn phasing were held constant. Permissive-only phasing was used since the capacities were easily determinable from HCM equations and the calibration could be applied to the PPLT mode. The variable parameters used for calibration were area type, cycle length, subject green ratio, and opposing volume, as these could be used in the HCM capacity calculations. All combinations of rural and urban area types, cycle lengths of $90 \mathrm{~s}$ and $120 \mathrm{~s}$, green ratios of 0.6 and 0.8 , and 100, 200, 400, 600, 800, 1,000, and 1,200 veh/hr/ln opposing volumes were used to create the 56 scenarios.

To calculate the capacity of each of the permitted left-turn scenarios, the following HCM 
equation was used:

$$
c_{p}=\frac{g_{u}}{C} s_{p}+\text { sneakers }
$$

where

$$
\begin{aligned}
& c_{p}=\text { capacity of the permissive left-turn (veh/hr), } \\
& g_{u}=\text { duration of permissive left-turn green time that is not blocked by an } \\
& \quad \text { opposing queue }(\mathrm{s}), \\
& C=\text { cycle length (s), and } \\
& s_{p}=\text { saturation flow rate of the permissive left-turn movement }(\mathrm{veh} / \mathrm{hr} / \mathrm{ln}) .
\end{aligned}
$$

Effectively, the equation determines the number of vehicles that are able to execute the permissive left-turn after the opposing queue clears and the number of vehicles in an hour that are sneakers, based on two sneakers per cycle. The duration of the permissive left-turn that is not blocked by the opposing queue (the unblocked green time), was calculated by deriving information from the queue polygon in the HCM that represented protected movements. In Figure 12 below, the amount of time that the opposing queue took to dissipate is represented by the variable $g_{s}(27)$. To determine this value for each scenario, the initial queue had to be divided by the rate at which the queue dissipated. 


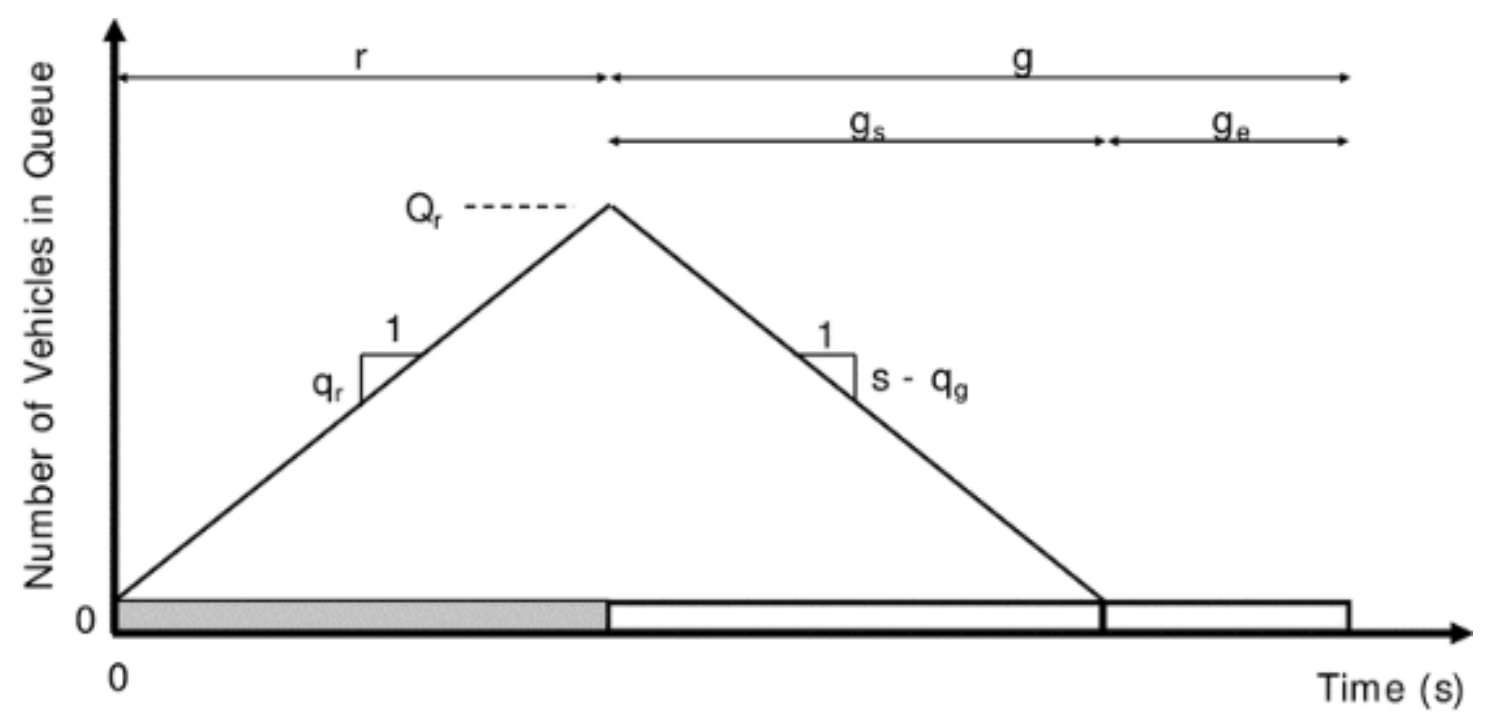

Figure 12 - Queue Accumulation Polygon for Protected Movements (27)

$$
g_{b}=g_{s}=\frac{Q_{r}}{s-q_{g}}=\frac{q_{r} r}{s-q_{g}}
$$

where

$$
\begin{aligned}
& g_{s}=\quad \text { queue service time }(\mathrm{s}) \text { or } g_{b}=\text { duration of permissive left-turn green time that } \\
& \quad \text { is not blocked by an opposing queue }(\mathrm{s}), \\
& Q_{r}=\text { queue size at the end of the effective red time }=q_{r} r(\text { veh }), \\
& q_{r}=\text { arrival flow rate during the effective red time }=\text { opposing volume }(\mathrm{veh} / \mathrm{hr} / \mathrm{ln}), \\
& r=\quad \text { effective red time }=C-g(\mathrm{~s}), \\
& g=\quad \text { effective subject street green time }=C \frac{G}{C}-l(\mathrm{~s}), \\
& \frac{G}{C}=\quad \text { subject street green ratio (decimal), } \\
& l=\quad \text { loss time, } 5 \text { assumed (s), } \\
& s=\quad \text { ideal saturation flow rate, } 1,900 \text { for urban and } 1,750 \text { for rural }(\mathrm{veh} / \mathrm{hr} / \mathrm{ln}),
\end{aligned}
$$




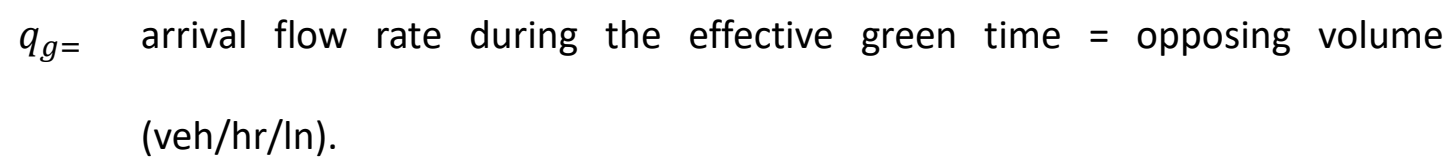

Once the blocked green time is determined, it can be subtracted by the effective green time for the subject street to calculate the unblocked green time, $g_{u}$. The saturation flow rate of the permissive left-turn approach, $s_{p}$, is determined from the HCM equation:

$$
s_{p}=\frac{v_{o} e^{-v_{o} t_{c g} / 3,600}}{1-e^{-v_{o} t_{f h} / 3,600}}
$$

where

$$
\begin{aligned}
& v_{o}=\text { opposing demand flow rate }=\mathrm{N} * \text { opposing volume }(\mathrm{veh} / \mathrm{h}), \\
& t_{c g}=\text { critical headway }=4.5(\mathrm{~s}) \text {, and } \\
& t_{f h}=\text { Follow-up headway }=2.5 \text { for exclusive lane }(\mathrm{s}) .
\end{aligned}
$$

Finally, the number of sneakers per hour is calculated by multiplying the value of two sneakers per cycle by the number of cycles in an hour:

$$
\text { sneakers }=\frac{3,600}{C} 2
$$

After the HCM-calculated permissive capacity corresponding to each scenario was determined, the values were compared to the outputs of one simulation run per scenario. Since the errors were larger than anticipated, changes to the base model had to be made to produce better results. The best method to achieve more accurate capacities was to alter the behavior of left-turning drivers when they approach conflict areas, as other parameters such as saturation flow rate had already been calibrated. In the VISSIM conflict areas, the "FrontGapDef" and "RearGapDef" attributes corresponding to the front and rear gap defaults for the turning vehicles were varied to determine values that would produce more accurate capacities. These values, as defined in the VISSIM User Manual, are the "time that a vehicle waits before entering the 
conflict area, after the vehicle with the right of way has left it" (28). By shortening or lengthening the time a left-turning vehicle waits to execute their turn, the number of vehicles served by each scenario can be changed, thus values of $0.3,0.4,0.5,0.6,0.7$, and 0.8 were tested for their effect, with both the front and rear gap matching one another in each scenario. From this, 280 new scenarios were run in the simulation (not including the original 56 scenarios with default values of 0.5 for the front and rear gap defaults). Again, each scenario was run once, and the errors were compiled over combinations of opposing volumes and area types for each value of front and rear gap default, the results of which can be found in Table 12 and Table 13.

Table 12 - Rural Calibration Percent Errors

\begin{tabular}{c|cccccc} 
Opposing & \multicolumn{7}{|c}{ Front and Rear Gap Default } \\
Volume & $\mathbf{0 . 3}$ & $\mathbf{0 . 4}$ & $\mathbf{0 . 5}$ & $\mathbf{0 . 6}$ & $\mathbf{0 . 7}$ & $\mathbf{0 . 8}$ \\
\hline 100 & $9 \%$ & $7 \%$ & $6 \%$ & $5 \%$ & $4 \%$ & $3 \%$ \\
200 & $10 \%$ & $6 \%$ & $\mathbf{4} \%$ & $2 \%$ & $-1 \%$ & $-2 \%$ \\
400 & $5 \%$ & $0 \%$ & $-3 \%$ & $-7 \%$ & $-10 \%$ & $-12 \%$ \\
600 & $-4 \%$ & $-9 \%$ & $-13 \%$ & $-17 \%$ & $-19 \%$ & $-22 \%$ \\
800 & $-9 \%$ & $-14 \%$ & $-18 \%$ & $-21 \%$ & $-23 \%$ & $-26 \%$ \\
1,000 & $-6 \%$ & $-9 \%$ & $-11 \%$ & $-12 \%$ & $-13 \%$ & $-14 \%$ \\
1,200 & $-15 \%$ & $-15 \%$ & $-15 \%$ & $-15 \%$ & $-15 \%$ & $-15 \%$
\end{tabular}

Table 13 - Urban Calibration Percent Errors

\begin{tabular}{c|cccccc} 
Opposing & \multicolumn{7}{|c}{ Front and Rear Gap Default } \\
Volume & $\mathbf{0 . 3}$ & $\mathbf{0 . 4}$ & $\mathbf{0 . 5}$ & $\mathbf{0 . 6}$ & $\mathbf{0 . 7}$ & $\mathbf{0 . 8}$ \\
\hline 100 & $15 \%$ & $13 \%$ & $12 \%$ & $10 \%$ & $9 \%$ & $8 \%$ \\
200 & $12 \%$ & $10 \%$ & $7 \%$ & $4 \%$ & $2 \%$ & $1 \%$ \\
400 & $4 \%$ & $0 \%$ & $-3 \%$ & $-7 \%$ & $-9 \%$ & $-12 \%$ \\
600 & $0 \%$ & $-6 \%$ & $-11 \%$ & $-16 \%$ & $-19 \%$ & $-22 \%$ \\
800 & $-9 \%$ & $-14 \%$ & $-20 \%$ & $-23 \%$ & $-26 \%$ & $-27 \%$ \\
1,000 & $-14 \%$ & $-17 \%$ & $-19 \%$ & $-21 \%$ & $-22 \%$ & $-24 \%$ \\
1,200 & $-15 \%$ & $-16 \%$ & $-16 \%$ & $-17 \%$ & $-17 \%$ & $-17 \%$
\end{tabular}


Though the simulation results did not perfectly match the theoretical HCM capacities, the most optimal front and rear gap values from the runs were selected. To produce better model performance, values were selected for two different levels of opposing volumes: 0.6 for opposing volumes of $400 \mathrm{veh} / \mathrm{hr} / \mathrm{ln}$ or less, and 0.3 for opposing volumes higher than $400 \mathrm{veh} / \mathrm{hr} / \mathrm{ln}$. Once the threshold values were selected, simulations were run 19 more times per scenario (56 scenarios) with the new values to ensure consistent results. This produced average HCM and simulated permissive left-turn capacities, as well as percent errors, below in Table 14 and a maximum of $10 \%$ variation across runs per scenario.

Table 14 - Final Calibration Results

\begin{tabular}{c|ccc|ccc}
$\begin{array}{c}\text { Opposing } \\
\text { Volume }\end{array}$ & HCM & $\begin{array}{c}\text { Rural } \\
\text { Simulation }\end{array}$ & \% Error & HCM & $\begin{array}{c}\text { Urban } \\
\text { Simulation }\end{array}$ & \% Error \\
\hline 100 & 827 & 877 & $6 \%$ & 829 & 925 & $12 \%$ \\
200 & 677 & 689 & $2 \%$ & 681 & 715 & $5 \%$ \\
400 & 448 & 413 & $-8 \%$ & 456 & 419 & $-8 \%$ \\
600 & 292 & 287 & $-2 \%$ & 302 & 289 & $-4 \%$ \\
800 & 186 & 173 & $-7 \%$ & 198 & 172 & $-13 \%$ \\
1,000 & 116 & 107 & $-8 \%$ & 128 & 106 & $-17 \%$ \\
1,200 & 85 & 74 & $-14 \%$ & 94 & 79 & $-16 \%$
\end{tabular}

\subsubsection{Experimental Design of Simulation Scenarios}

Rather than a full-factorial experimental design that would produce millions of simulation scenarios to run, a sliced Latin Hypercube Design (LHD) was used to determine the combinations of model parameters to simulate. A LHD with $\mathrm{N}$ variables produces $\mathrm{M}$ random samples from $\mathrm{M}$ equally-probable intervals for each $\mathrm{N}$. In doing so, exactly one point is selected from each interval of each variable. These values were then permuted to ensure random combinations of the variables, rather than each variable following the same trend (i.e. as variable 
A increases, so does variable B).

An illustration of a sampling of two of the simulation parameters using a LHD can be seen below in Figure 13, and an illustration of a full-factorial sampling is shown in Figure 14. In both cases, 25 samples are chosen from the variables opposing volume and average speed. Using the LHD sampling, only one sample can occur in an interval (between the gridlines) in each variable, and the points are scattered across the array. For opposing volume, intervals of size 40 were used $((\max -\min ) / \mathrm{M}=(1,200-200) / 25)$, and for average speed, intervals of size 0.8 were used ((55-35)/25). In the full-factorial sampling, five levels of each variable were used, and each combination of the levels was plotted. As evident by the two graphs, the LHD produces a random scatter of samples that can be modeled as continuous variables, whereas the full-factorial design uses discrete levels of the variables. In addition to the continuous nature of the variables, LHD produces fewer combinations with similar modeling accuracy, as the number of samples can be controlled, with a general rule of thumb being 10 observations per dimension (29). In a full-factorial design, the number of samples increases exponentially with the number of dimensions, creating considerably more scenarios to achieve the same result. 


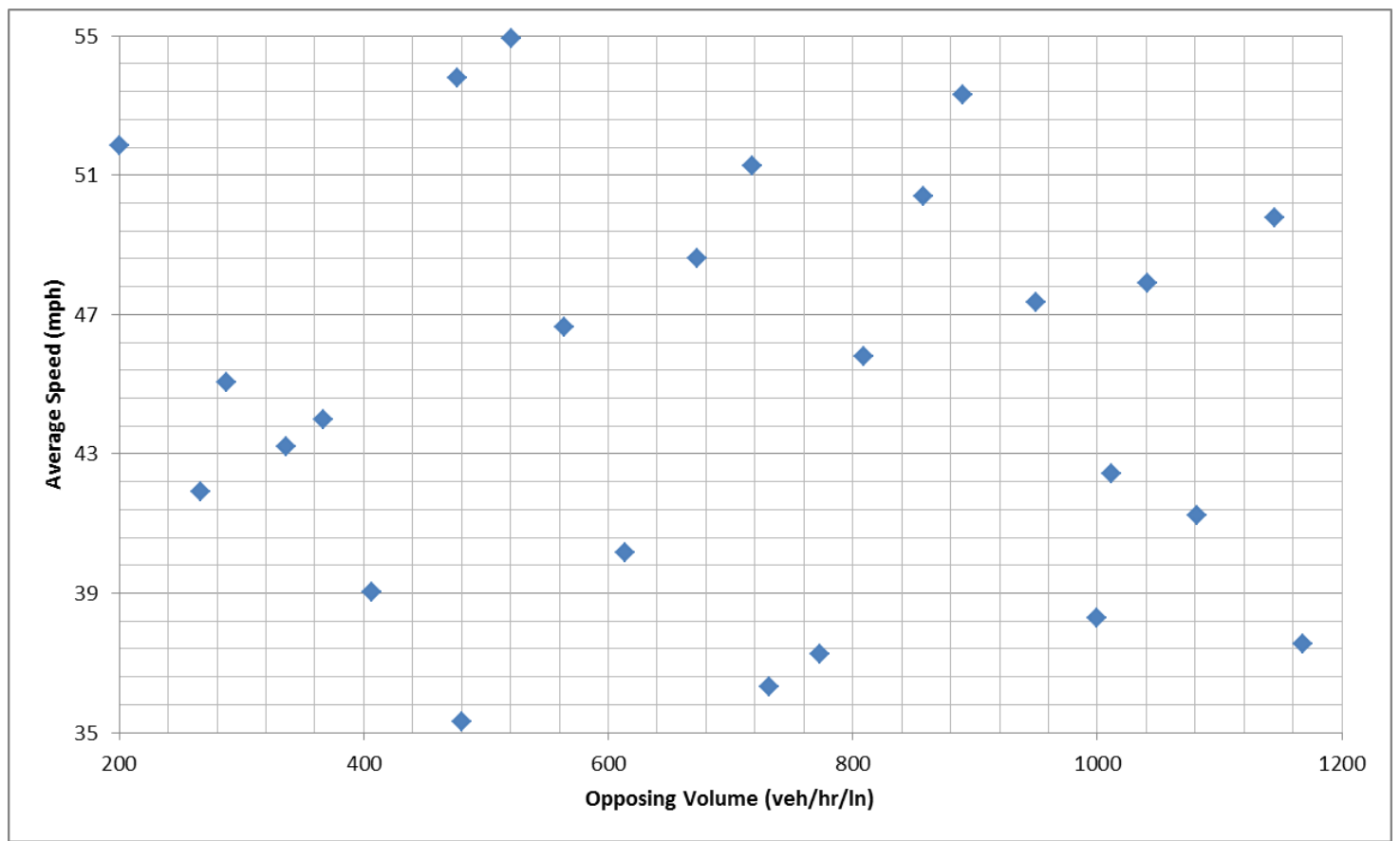

Figure 13 - Example LHD with 25 Samples

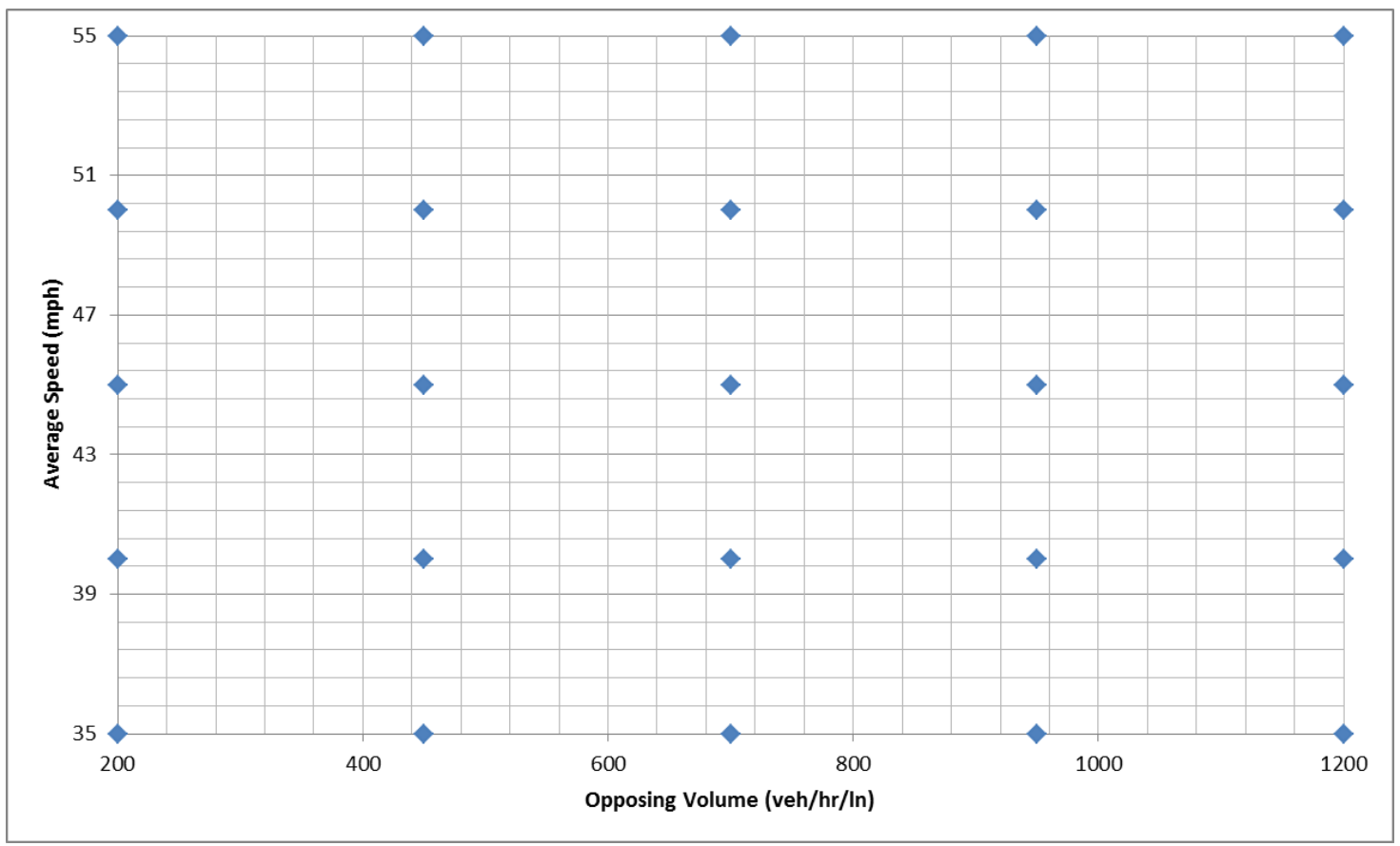

Figure 14 - Example Full-Factorial Design with 25 Samples 
LHD's require all variables to be continuous; therefore, a sliced LHD had to be used in developing the simulation scenarios, as there were three discrete variables that needed to be modeled (area type, number of opposing lanes, and left-turn mode). In a sliced LHD, a Latin hypercube is created for each combination of discrete variables, considered a slice. For the purpose of the research, 30 slices were created ( 2 levels of area type $x 3$ levels of opposing lanes x 5 levels of left-turn mode). In each slice, 25 combinations of the continuous variables were determined using the aforementioned procedure for LHDs. A value of 25 combinations was considered to be sufficient for the purpose of the simulations as it provided the required degree of freedom for a quadratic response surface and provided an acceptable number of observations per dimension (29). As a result, 750 unique combinations of the seven simulation parameters were used in VISSIM.

\subsubsection{VISSIM Model Execution}

Once the VISSIM model was calibrated and simulation parameter combinations were selected, simulation runs could be executed. Each of the 750 scenarios was run ten times each in VISSIM using a script developed by another researcher to automate the process. It was decided that ten runs would be sufficient, as during the calibration process the minimum sample size for each of the scenarios to which the model was calibrated was calculated at a 95\% confidence level using the average and standard deviation of the results of 20 runs. As the model runs completed, capacity results were compiled in a spreadsheet for each of the 7,500 simulation runs. Additionally, TRJ files were produced by VISSIM for each run and saved to be analyzed for conflicts in SSAM. 


\subsubsection{SSAM Conflict Analysis}

Since VISSIM models safe driving behaviors, traditional measures of safety could not be used in the evaluation of the models; therefore, surrogate safety measures had to be used, in particular Time to Collision (TTC) and Post Encroachment Time (PET). TTC is defined as the time remaining until two vehicles would come in contact with one another, based on their trajectories, if they did not alter their paths. PET is the time that elapsed between one vehicle crossing a reference point and the second vehicle crossing that same point. Both of these values help to indicate potential conflicts between vehicles.

To determine the number of vehicles that encountered potential conflicts in each simulation run, the Federal Highway Administration's (FHWA) Surrogate Safety Assessment Model (SSAM) was used to process TRJ files from VISSIM. Using the TRJ files, SSAM models each vehicle's trajectory throughout the simulation to determine minimum TTC and PET values for each vehicle's interactions with other vehicles. In doing so, SSAM classifies conflicts as three types: Crossing, Rear End, and Lane Change, based on conflict angles, shown below in Figure 15 (30). When evaluating the TRJ files, SSAM classifies conflicts based on user-defined thresholds. Maximum TTC, maximum PET, rear end conflict angle, and crossing conflict angle were set at 2, 5, 30, and 80, respectively, for the purposes of the research, as these were the default values suggested by the software. Once SSAM processes all of the vehicle trajectories and determines conflicts, the results can be exported as a CSV file. This file lists conflicts as separate rows, with the columns indicating information about each conflict such as the TRJ file name, minimum TTC, minimum PET, conflict type, lane information, etc. 

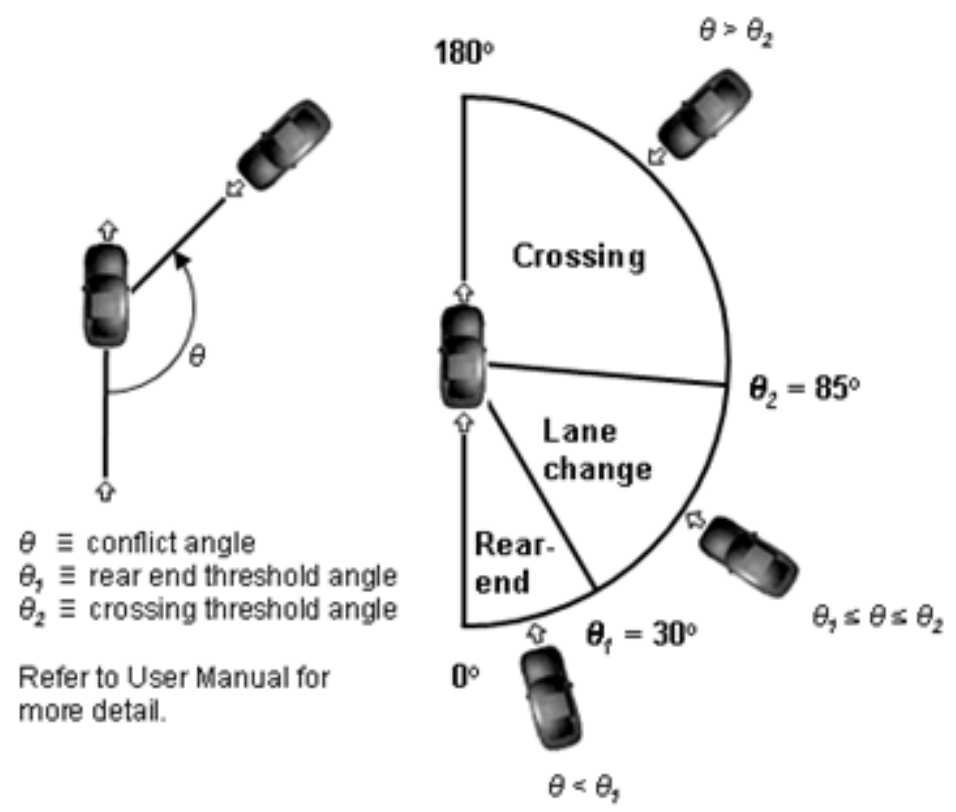

Figure 15 - SSAM Conflict Angles (30)

Once the 780 GB of TRJ files were processed with SSAM, roughly two to three million conflicts resulted. Since the target conflict type for left-turns was the crossing conflict type, rear end and lane change conflicts had to be removed from the files. To do this, an executable Java program was developed to scan through each CSV file and remove lines that corresponded to non-crossing conflict types. As a result, roughly 860,000 crossing conflicts were found, though over $90 \%$ had TTC or PET values of 0 , which would be considered "crashes." Since crashes cannot occur in VISSIM, these values were decided to be errors in the SSAM processing and were removed, as is suggested in the SSAM Final Report (30). Additionally, it was observed that in many instances, vehicle ID's were found in more than one conflict. Since only one conflict record should exist per turning vehicle, duplicates were removed, resulting in a total of 64,000 final, filtered conflicts across the 7,500 simulation runs.

The final step in the post-processing of the SSAM results was to determine the number of conflicts for each simulation run, using different TTC and PET thresholds. A final Excel 
workbook was created that included capacity and conflict data from the VISSIM and SSAM models at two levels: a simulation run level (7,500 runs) and an aggregate scenario-level (750 scenarios). Data in both spreadsheets included VISSIM simulation parameters, capacities, and counts of conflicts with TTC less than or equal to $0.5,1,1.5$, and 2 , as well as counts of conflicts with PET less than or equal to $2.5,3,3.5,4$, and 4.5. For the simulation run level there were 7,500 rows of data corresponding to each of the ten runs for the 750 scenarios. The scenariolevel sheet had 750 rows of data corresponding to average values of the number of conflicts and capacities for the ten runs of each scenario. Ultimately, the aggregate data across each of the simulation iterations for separate scenarios were used for further analysis.

\subsubsection{Capacity and Conflict Prediction Model Development}

With the simulated capacity and conflict results from VISSIM and SSAM, along with the parameters used for each result, multiple regression analysis was performed to create models to predict capacity and conflicts based on different combinations of variables. Separate models were developed for capacity and conflicts for both permissive-only and PPLT control modes using IBM's SPSS Statistics software package. Additionally, the data for each left-turn mode was separated into two sets of data each: cases with non-zero permissive capacities and cases with no permissive capacity (i.e. sneakers only for permissive-only phasing). The following sections describe how the models were created and validated.

\subsubsection{Separation of Datasets}

Two sets of data were created for both PPLT and permissive-only capacity and conflict results to represent cases with non-zero permissive capacities and cases with zero permissive 
capacity. This was done to separate cases that produced zero conflicts from those that produced non-zero conflicts so that models would fit the data better. Additionally, with zero vehicles turning during the permissive portion of PPLT or as non-sneakers for permissive-only, no conflicts were expected; therefore, zero conflicts could be assumed with zero permissive capacity, yet the conflicts for the other cases still had to be modeled.

Thresholds were determined separately for permissive-only and PPLT phasing modes to determine to which case a simulation scenario belonged. Beginning with the permissive-only left-turn phasing, the percentage of the capacity that was sneakers was calculated for each scenario, using Equation 8 in the Model Calibration section of this document to determine the theoretical number of sneakers per scenario. The data was then sorted vertically from greatest to least percentage of sneakers, and then classified as a "sneakers-only" case or not. The determination as to whether a scenario produced only sneakers was made by classifying any scenario with greater than a certain percentage of its capacity coming from sneakers as a sneakers-only case. This threshold was determined based on the existence of a clear break point in the percent sneaker capacity data. Additionally, the average number of conflicts per scenario for each of the TTC and PET thresholds was evaluated to determine which threshold conformed to the fact that zero conflicts should occur when zero vehicles are able to execute a permissive left-turn.

Characteristics of the sneakers-only cases were then analyzed to determine combinations of values of the simulation parameters that set these cases apart from the non-sneakers-only cases. As opposing volume was a continuous variable and is accepted as one of the most influential factors in determining permissive capacities, it was used as a threshold value for different combinations of other variables. Two sets of thresholds were developed and compared 
for their ability to correctly predict whether a scenario was a non-zero permissive capacity case or not, and the most optimal set of thresholds was chosen based on simplicity of the thresholds and prediction ability. A similar threshold development approach was used for the PPLT scenarios. For these cases, instead of using the percentage of the capacity that were sneakers, as PPLT control mode also has protected portion capacities that produce zero conflicts, the number of conflicts in a scenario was used as a metric since it was common between the two sets of leftturn modes. Once each scenario was classified as either a zero-permissive capacity or a non-zero permissive capacity from the number of conflicts, characteristics of the two sets were compared, as was done with the permissive-only dataset. Again, the opposing volume was used as a threshold value for different combinations of other simulation parameters.

\subsubsection{Model Development Process in SPSS}

After datasets were created for the combinations of permissive-only phasing, PPLT phasing, zero permissive capacities, and non-zero permissive capacities, more accurate prediction models could be created than for the permissive-only and PPLT models with one set of data each. Additionally, it should be noted that the conflict prediction models used normalized conflicts, with the scenario's left-turn capacity as the method of exposure, to produce the number of conflicts per 100 turning vehicles. With the models predicting number of conflicts per 100 left-turning vehicles, the number of conflicts for a particular scenario can be calculated by multiplying the prediction by the left-turn demand, divided by 100 . For each of the sets of data, roughly $70-80 \%$ of the data was randomly selected as the training dataset and the remaining data as the validation set. The following sections outline the steps taken in developing the five models in SPSS. 


\subsection{Analysis of Variance (ANOVA)}

To determine which parameters were significant in predicting the capacities or conflicts for a dataset, Analysis of Variance (ANOVA) was performed using a univariate general linear model in SPSS. All continuous variables and discrete variables (area type, number of opposing lanes, and the protected green ratio for PPLT) were entered as either covariates or fixed factors, respectively, and the dependent variable was set to either capacity or conflicts per 100 turning vehicles. Once the software processed the inputs, significance levels of each parameter were evaluated. Parameters that had significance values of less than 0.05 , corresponding to the $95 \%$ confidence level, were noted, and further used in the analysis to develop the models. As is the convention for ANOVA, parameters whose significance values were higher than 0.05 were discarded, as they were not statistically significant predictors for the particular model.

\subsection{Stepwise Regression}

Significant parameters were further determined through forward stepwise regression. In this step, the statistically significant predictor variables found from ANOVA were entered as input variables and either capacity or the number of conflicts per 100 turning vehicles was set as the dependent variable. Using stepwise regression, parameters are entered into a prediction model in order of their contribution, with the variables with the highest contribution to the model being entered first. This produces multiple models that each have adjusted $r$-squared $\left(\mathrm{R}^{2}\right)$ values associated with them. Adjusted $\mathrm{R}^{2}$ values were compared to determine whether adding a particular parameter into the model made the predictions more accurate. Typically, the last variable added to the model was evaluated as to whether or not it made enough of a contribution 
to the model fit to justify a more complex model. If the increase in $\mathrm{R}^{2}$ was not justifiable, the parameter was removed from further model construction

Potential transformations of the significant continuous variables were also explored. This was done by using the "Curve Estimation" tool in SPSS, which fits several curves including a logarithmic, inverse, quadratic, power, and exponential curve, among others, to the single variable. For each continuous variable, up to two different curves (not including linear) and their parameters were chosen to be analyzed in the regression models; these curves typically were those with the highest $\mathrm{R}^{2}$ values.

\subsection{Linear Regression and Model Selection}

The final step in creating predictions for capacity and number of conflicts per 100 leftturning vehicles was the development of several models and selecting the model that fit the data the best, while avoiding violating regression assumptions. Once each of the linear regression models was created in SPSS, adjusted $\mathrm{R}^{2}$ values were compared against one another, expected cumulative probability vs. observed cumulative probability (P-P) plots were checked for linearity, standardized residuals were checked for a normal distribution, Variance Inflation Factors (VIF) were checked to ensure multicollinearity was not an issue, and parameter coefficients were checked to make sure they were logical in sign and order of magnitude. Additionally, for each model, the residuals for each continuous variable were plotted against the variable to check for linearity, homoscedasticity, and independence of errors. Of all the models, the optimal model was selected as the model that fit the data best while conforming to the regression assumptions, though in some cases the increase in model fit over another model outweighed the marginal decrease in regression assumption conformity. 


\subsection{Model Validation}

After selecting the best model form for each set of data, the models were reconstructed with $70-80 \%$ of the overall data using the selected model forms, and validated with the remaining data to ensure the models were not overfit to the data used in construction. The validation was completed by calculating the respective predicted capacities or number of conflicts per 100 left-turning vehicles for each model using the entire dataset (including the training and validation datasets). These predictions were then compared to the actual simulated capacitates or number of conflicts and aggregated based on the dataset: build or validation data. Statistics such as mean square prediction error, mean absolute error, mean absolute percent error, and mean bias were compared across the training and validation datasets to ensure each produced a similar result.

\subsection{RESULTS}

\subsubsection{Separation of Datasets}

To create as few models as possible, it was first attempted to create two capacity and two conflict models (one each for permissive-only and PPLT). Following the same process as described in the model development section of this chapter, these models were found to not fit the data well and violated regression assumptions; therefore, it was decided to create several more specific models for the data.

In separating the datasets, the determination as to whether a scenario produced only sneakers for the permissive-only mode was made by classifying any scenario with greater than $90 \%$ of its capacity coming from sneakers as a sneakers-only case, as there was a clear break in 
the data (i.e. there were many scenarios with $90 \%$, one in the $80 \%-90 \%$ range, and the rest of the data less than $80 \%$ ). Additionally, it was determined that the TTC less than or equal to 2 cases for conflicts matched the assumption that when there are zero permissive capacity zero conflicts should occur. Because of this, the average number of conflicts with TTC less than or equal to 2 was selected as the average number of conflicts per scenario for all of the models.

Using a threshold value of opposing volume, two sets of thresholds were determined to produce a $1 \%$ and a $2 \%$ prediction error for predicting whether a scenario was a sneakers-only case. The threshold set that produced a $1 \%$ prediction error was based on combinations of the number of opposing lanes and the subject street green ratio, rounded to the nearest tenth, and the set that produced a $2 \%$ prediction error was purely based on the subject street green ratio, rounded to the nearest tenth. Since removing the number of opposing lanes criteria for the threshold only produced a $1 \%$ increase in prediction error, the more simplified threshold set was selected, seen below in Table 15. These thresholds established the maximum opposing volumes, inclusive of the value, to which non-zero permissive capacities can be realized, based on the subject street's percentage of the signal cycle.

\section{Table 15 - Sneakers-Only Thresholds for Permissive-Only}

\begin{tabular}{c|c}
$\begin{array}{c}\text { Subject } \\
\begin{array}{c}\text { Street Green } \\
\text { Ratio }\end{array}\end{array}$ & $\begin{array}{c}\text { Opposing } \\
\text { Volume } \\
\text { (veh/hr/ln) }\end{array}$ \\
\hline 0.3 & 450 \\
0.4 & 625 \\
0.5 & 875 \\
0.6 & 900 \\
0.7 & 1,000 \\
0.8 & 1,100
\end{tabular}

In determining the thresholds for the PPLT scenarios, the greater than sneakers-only cases 
corresponded to an average number of conflicts of 2.3 or greater was carried over from the permissive-only scenarios to establish the cases where capacities were greater than just protected and sneaker capacities.

The set of thresholds for PPLT that was found to produce the most accurate result with the fewest combinations of parameters was the combination of protected green ratio and subject street green ratio. Like with the permissive-only dataset, the thresholds established maximum opposing volumes, inclusive of the value, to which non-zero permissive capacities are found, based on the subject street's and the subject left-turn's protected portion percentage of the signal cycle, found in Table 16. 
Table 16 - Zero-Permissive Capacity Thresholds for PPLT

\begin{tabular}{c|c|c}
$\begin{array}{c}\text { Protected } \\
\text { Green } \\
\text { Ratio }\end{array}$ & $\begin{array}{c}\text { Subject } \\
\text { Street Green } \\
\text { Ratio }\end{array}$ & $\begin{array}{c}\text { Opposing } \\
\text { Volume } \\
\text { (veh/hr/ln) }\end{array}$ \\
\hline \multirow{5}{*}{0.1} & 0.3 & 250 \\
& 0.4 & 450 \\
& 0.5 & 625 \\
& 0.6 & 825 \\
& 0.7 & 975 \\
0.15 & 0.8 & 975 \\
\hline & 0.3 & 0 \\
& 0.4 & 300 \\
& 0.5 & 550 \\
& 0.6 & 700 \\
& 0.7 & 925 \\
0.2 & 0.8 & 975 \\
\hline \multirow{5}{*}{0.25} & 0.3 & 0 \\
& 0.4 & 0 \\
& 0.5 & 475 \\
& 0.6 & 625 \\
& 0.7 & 700 \\
& 0.8 & 900 \\
\hline & 0.3 & 0 \\
& 0.4 & 0 \\
& 0.5 & 400 \\
& 0.6 & 525 \\
& 0.8 & 600 \\
& & 900 \\
& 0.7 &
\end{tabular}

\subsubsection{Left-Turn Capacity Models}

Three left-turn capacity prediction models were created using SPSS, corresponding to non-zero permissive capacity cases of PPLT, zero permissive capacity cases of PPLT, and nonzero permissive capacity cases of permissive-only phasing. The zero-permissive capacity cases 
(sneakers-only cases) for permissive-only phasing were not modeled, as capacities for these cases could be calculated using Equation 8. For each of the models, regression assumptions were conformed to, good model fit was found, and the models were validated.

\subsubsection{PPLT Capacity Model for Non-Zero Permissive Capacities}

As shown in Table 17, ANOVA results indicated that the discrete variables for area type, opposing lanes, and the protected green ratios, as well as continuous variables of cycle length, green ratio, opposing volume, and speed had a statistically significant impact on capacity, as they had significance values less than 0.05 . 
Table 17 - ANOVA Results for PPLT Capacity Model for Non-Zero Permissive Capacities

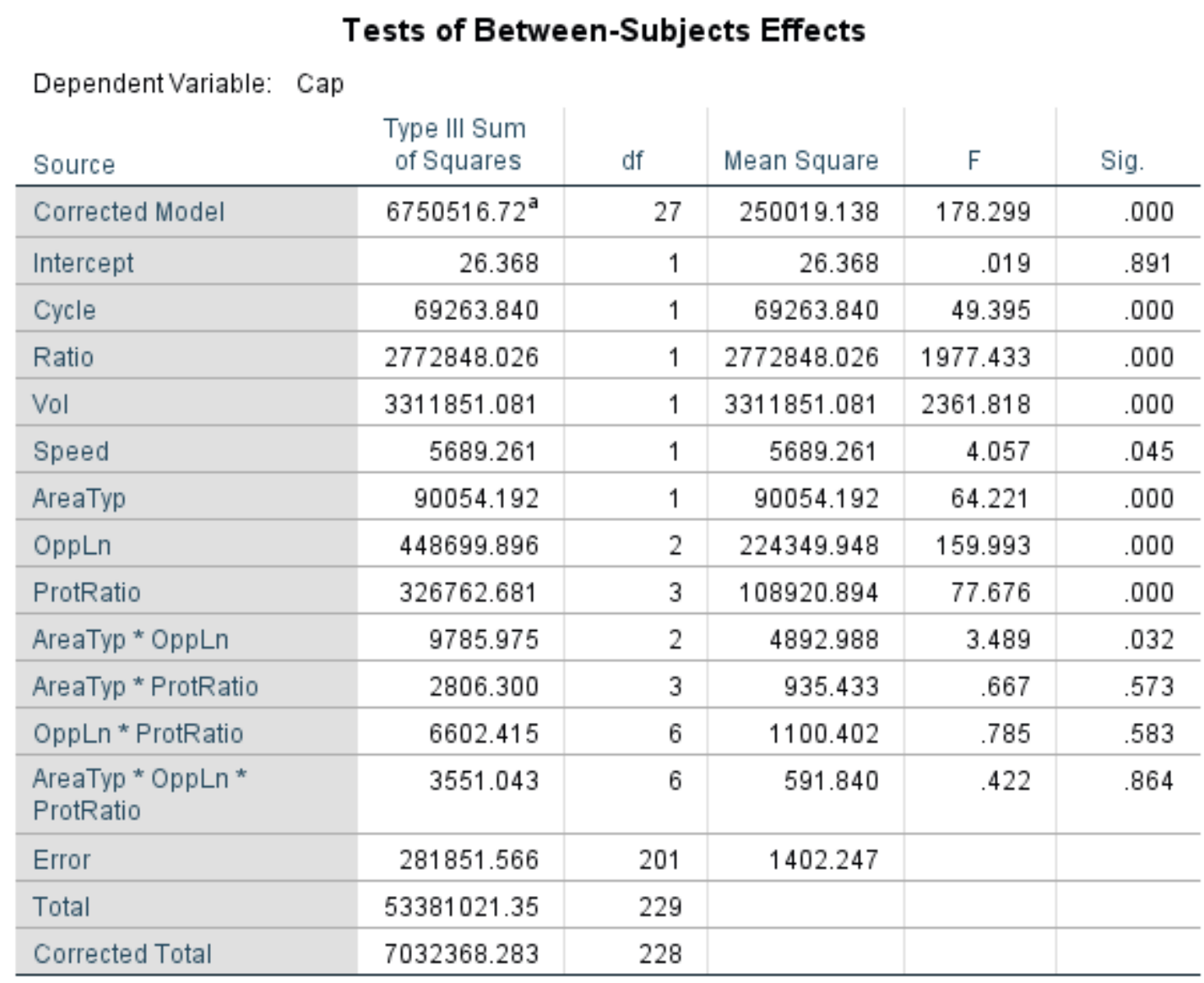

a. $\mathrm{R}$ Squared $=.960($ Adjusted $\mathrm{R}$ Squared $=.955)$

Following the ANOVA analysis, models for predicting capacity were developed. Although speed was a variable found to be statistically significant, it was later removed from the model, as it did not add value when stepwise regression was performed. Equation 9 shows the PPLT capacity when permissive capacity is non-zero. The final model that provided the best fit for the data for this dataset included dummy variables for area type (URB), one lane configurations (LN1), two lane configurations (LN2), PPLT with 10\% protected ratios (PP10), PPLT with 15\% protected ratios (PP15), and PPLT with 20\% protected ratios (PP20). Additionally, green ratio, opposing volume, and the inverse of cycle length were used as the 
significant continuous variables. The coefficients for the parameters of the aforementioned model parameters, along with the constant factor for the model can be found in the second column of Table 18 below. An adjusted $\mathrm{R}^{2}$ value of 0.956 resulted from this model form, providing good fit with the variables included. For the validation phase of the finalization of this model, mean square prediction error (MSPE), mean absolute error (MAE), mean absolute percent error (MAPE), and mean bias were calculated for both the training and validation datasets, shown in Table 19. The model validation showed acceptable results, with similar and reasonable error levels.

$$
\begin{aligned}
c=128.5+ & (39.6 * U R B)+(120.2 * L N 1)+(54.0 * L N 2) \\
& -(109.8 * P P 10)-(66.21 * P P 15)-(33.51 * P P 20) \\
& -\left(\frac{10540}{C}\right)+\left(1119 * \frac{G}{C}\right)-(0.7103 * q)
\end{aligned}
$$

where

$$
\begin{gathered}
c=\quad \text { left-turn capacity (veh/hr) } \\
U R B=\text { area type dummy variable (1 for urban), } \\
L N 1=\text { one opposing lane dummy variable (1 for one opposing lane), } \\
L N 2=\text { two opposing lanes dummy variable (1 for two opposing lanes), } \\
P P 10=\text { PPLT with } 0.10 \text { protected ratio dummy variable, } \\
P P 15=\text { PPLT with } 0.15 \text { protected ratio dummy variable, } \\
P P 20=\text { PPLT with } 0.20 \text { protected ratio dummy variable, } \\
C=\quad \text { cycle length (s), } \\
\frac{G}{C}=\quad \text { subject street green ratio (decimal), and } \\
q=\quad \text { opposing flow rate (opposing volume) (veh } / \mathrm{hr} / \mathrm{ln}) .
\end{gathered}
$$


Table 18 - Regression Parameters for PPLT Capacity Model for Non-Zero Permissive Capacities

\begin{tabular}{|c|c|c|c|c|c|c|c|c|}
\hline \multicolumn{9}{|c|}{ Coefficients $^{a}$} \\
\hline & & \multicolumn{2}{|c|}{ Unstandardized Coefficients } & \multirow{2}{*}{$\begin{array}{c}\text { Standardized } \\
\text { Coefficients } \\
\text { Beta }\end{array}$} & \multirow[b]{2}{*}{$t$} & \multirow[b]{2}{*}{ Sig. } & \multicolumn{2}{|c|}{ Collinearity Statistics } \\
\hline \multicolumn{2}{|c|}{ Model } & $\mathrm{B}$ & Std. Error & & & & Tolerance & VIF \\
\hline \multirow[t]{10}{*}{1} & (Constant) & 128.507 & 20.760 & & 6.190 & .000 & & \\
\hline & URB & 39.557 & 5.558 & .112 & 7.118 & .000 & .991 & 1.009 \\
\hline & LN1 & 120.150 & 6.938 & .332 & 17.318 & .000 & .668 & 1.497 \\
\hline & LN2 & 54.042 & 7.185 & .144 & 7.522 & .000 & .671 & 1.491 \\
\hline & PP10 & -109.765 & 9.084 & -.297 & -12.083 & .000 & .407 & 2.459 \\
\hline & PP15 & -66.205 & 8.996 & -.169 & -7.359 & .000 & .463 & 2.158 \\
\hline & PP20 & -33.514 & 9.471 & -.077 & -3.539 & .001 & .516 & 1.937 \\
\hline & Cyclelnv & -10544.478 & 1322.935 & -.126 & -7.971 & .000 & .986 & 1.014 \\
\hline & Ratio & 1118.851 & 27.603 & .733 & 40.533 & .000 & .750 & 1.333 \\
\hline & Vol & -.710 & .016 & -.815 & -44.670 & .000 & .735 & 1.360 \\
\hline
\end{tabular}

a. Dependent Variable: Cap

Table 19 - Validation Results for PPLT Capacity Models for Non-Zero Permissive Capacities

\begin{tabular}{cccccc} 
Dataset & N & MSPE & MAE & MAPE & Mean Bias \\
\hline Build & 182 & 1316.1 & 27.33 & $6.02 \%$ & 0.00 \\
Validation & 47 & 1385.6 & 30.42 & $6.98 \%$ & 1.23
\end{tabular}

\subsubsection{PPLT Capacity Model for Zero-Permissive Capacities}

The ANOVA results for the PPLT model when permitted capacity is zero is shown in Table 20. The fixed variables for area type, protected green ratio, and the continuous variable for cycle length were all found to have a statistically significant effect on capacity. 
Table 20 - ANOVA Results for PPLT Capacity Model for Zero-Permissive Capacities

\section{Tests of Between-Subjects Effects}

Dependent Variable: Cap

\begin{tabular}{lr|r|r|r|r}
\hline Source & $\begin{array}{c}\text { Type III Sum } \\
\text { of Squares }\end{array}$ & df & Mean Square & \multicolumn{1}{c}{ F } & \multicolumn{1}{c}{ Sig. } \\
\hline Corrected Model & $3687675.47^{\text {a }}$ & 27 & 136580.573 & 1370.517 & .000 \\
\hline Intercept & 410316.938 & 1 & 410316.938 & 4117.322 & .000 \\
\hline Cycle & 1065.461 & 1 & 1065.461 & 10.691 & .001 \\
\hline Ratio & 297.362 & 1 & 297.362 & 2.984 & .085 \\
\hline Vol & 234.946 & 1 & 234.946 & 2.358 & .126 \\
\hline Speed & 104.247 & 1 & 104.247 & 1.046 & .307 \\
\hline AreaTyp & 40500.944 & 1 & 40500.944 & 406.406 & .000 \\
\hline OppLn & 198.463 & 2 & 99.232 & .996 & .371 \\
\hline ProtRatio & 3427748.641 & 3 & 1142582.880 & 11465.238 & .000 \\
\hline AreaTyp * OppLn & 42.755 & 2 & 21.378 & .215 & .807 \\
\hline AreaTyp * ProtRatio & 5556.258 & 3 & 1852.086 & 18.585 & .000 \\
\hline OppLn * ProtRatio & 59.982 & 6 & 9.997 & .100 & .996 \\
\hline $\begin{array}{l}\text { AreaTyp * OppLn * } \\
\text { ProtRatio }\end{array}$ & 350.959 & 6 & 58.493 & .587 & .741 \\
\hline Error & 34182.101 & 343 & 99.656 & & \\
\hline Total & 44946635.39 & 371 & & & \\
\hline Corrected Total & 3721857.573 & 370 & & & \\
\hline
\end{tabular}

a. R Squared $=.991$ (Adjusted R Squared $=.990$ )

The results of the stepwise regression for the capacity model are shown in Equation 10. This model form produced a 0.990 adjusted $\mathrm{R}^{2}$ value with the variables included. Table 21 also shows the parameter coefficients, as well as the constant in the model. Finally, Table 22 lists the results from validating the model for this dataset. Validation results again showed good performance, as each of the statistics were within reason of each other between to two datasets. Additionally, the percent error values for both sets were the same.

$$
\begin{aligned}
c=406.5+ & (22.10 * U R B)-(275.0 * P P 10)-(179.6 * P P 15) \\
- & (89.09 * P P 20)+(0.5015 * C)-\left(0.00166 * C^{2}\right)
\end{aligned}
$$


Table 21 - Regression Parameters for PPLT Capacity Model for Zero-Permissive Capacities

\begin{tabular}{|c|c|c|c|c|c|c|c|c|}
\hline \multicolumn{9}{|c|}{ Coefficients $^{a}$} \\
\hline \multirow[b]{2}{*}{ Model } & & \multicolumn{2}{|c|}{ Unstandardized Coefficients } & \multirow{2}{*}{$\begin{array}{c}\text { Standardized } \\
\text { Coefficients } \\
\text { Beta }\end{array}$} & \multirow[b]{2}{*}{$t$} & \multirow[b]{2}{*}{ Sig. } & \multicolumn{2}{|c|}{ Collinearity Statistics } \\
\hline & & B & Std. Error & & & & Tolerance & VIF \\
\hline \multirow[t]{7}{*}{1} & (Constant) & 406.484 & 7.327 & & 55.477 & .000 & & \\
\hline & URB & 22.104 & 1.181 & .109 & 18.711 & .000 & .990 & 1.010 \\
\hline & PP10 & -274.969 & 1.714 & -1.081 & -160.404 & .000 & .745 & 1.343 \\
\hline & PP15 & -179.552 & 1.617 & -.760 & -111.016 & .000 & .721 & 1.386 \\
\hline & PP20 & -89.093 & 1.574 & -.391 & -56.618 & .000 & .710 & 1.409 \\
\hline & Cycle & .502 & .097 & .231 & 5.188 & .000 & .017 & 58.761 \\
\hline & Cycle2 & -.002 & .000 & -.245 & -5.507 & .000 & .017 & 58.746 \\
\hline
\end{tabular}

a. Dependent Variable: Cap

Table 22 - Validation Results for PPLT Capacity Models for Zero-Permissive Capacities

\begin{tabular}{cccccc} 
Dataset & N & MSPE & MAE & MAPE & Mean Bias \\
\hline Build & 290 & 97.8 & 7.68 & $2.33 \%$ & -0.01 \\
Validation & 81 & 109.9 & 8.01 & $2.33 \%$ & 0.28
\end{tabular}

\subsubsection{Permissive-Only Capacity Model for Non-Zero Permissive Capacities}

The ANOVA results for permitted phasing where the permitted capacity is greater than zero are shown in Table 23. Discrete variables for area type, opposing lanes, and the continuous variables for green ratio and opposing volume produced statistically significant impacts on mean capacity. 
Table 23 - ANOVA Results for Permissive-Only Capacity Model for Non-Zero Permissive Capacities

\begin{tabular}{|c|c|c|c|c|c|}
\hline \multicolumn{6}{|c|}{ Tests of Between-Subjects Effects } \\
\hline \multicolumn{6}{|c|}{ Dependent Variable: Cap } \\
\hline Source & $\begin{array}{c}\text { Type III Sum } \\
\text { of Squares }\end{array}$ & df & Mean Square & $\mathrm{F}$ & Sig. \\
\hline Corrected Model & $2885258.99^{\mathrm{a}}$ & 9 & 320584.332 & 174.293 & .000 \\
\hline Intercept & 1172.111 & 1 & 1172.111 & .637 & .427 \\
\hline Cycle & 3165.920 & 1 & 3165.920 & 1.721 & .193 \\
\hline Ratio & 1356081.367 & 1 & 1356081.367 & 737.267 & .000 \\
\hline Vol & 1983795.271 & 1 & 1983795.271 & 1078.538 & .000 \\
\hline Speed & 5120.861 & 1 & 5120.861 & 2.784 & .099 \\
\hline AreaTyp & 14349.080 & 1 & 14349.080 & 7.801 & .006 \\
\hline OppLn & 431797.157 & 2 & 215898.579 & 117.378 & .000 \\
\hline AreaTyp * OppLn & 1141.216 & 2 & 570.608 & .310 & .734 \\
\hline Error & 167379.643 & 91 & 1839.337 & & \\
\hline Total & 10323058.86 & 101 & & & \\
\hline Corrected Total & 3052638.630 & 100 & & & \\
\hline
\end{tabular}

a. R Squared $=.945$ (Adjusted R Squared $=.940$ )

The final model that provided the best fit for this dataset used dummy variables for area type and number of opposing lanes, as well as continuous variables for subject street green ratio (squared) and opposing volume. The coefficients for the significant parameters can be found in Table 24, and the equation for the model can be found below in Equation 11. The model described for this dataset had an adjusted $\mathrm{R}^{2}$ value of 0.926 , and the validation results are shown in Table 25. Validation results were within 13 percent, and the validation data set proved to fit the models better in all categories except for mean bias, which showed a 16-vehicle underprediction overall.

$$
\begin{aligned}
c=246.2+ & (26.05 * U R B)+(161.8 * L N 1)+(64.77 * L N 2) \\
& +\left(844.4 *\left(\frac{G}{C}\right)^{2}\right)-(0.6788 * q)
\end{aligned}
$$


Table 24 - Regression Parameters for Permissive-Only Capacity Model for Non-Zero Permissive Capacities

\begin{tabular}{|c|c|c|c|c|c|c|c|c|}
\hline \multicolumn{9}{|c|}{ Coefficients $^{a}$} \\
\hline \multirow[b]{2}{*}{ Model } & & \multicolumn{2}{|c|}{ Unstandardized Coefficients } & \multirow{2}{*}{$\begin{array}{c}\text { Standardized } \\
\text { Coefficients } \\
\text { Beta }\end{array}$} & \multirow[b]{2}{*}{$t$} & \multirow[b]{2}{*}{ Sig. } & \multicolumn{2}{|c|}{ Collinearity Statistics } \\
\hline & & B & Std. Error & & & & Tolerance & VIF \\
\hline \multirow[t]{6}{*}{1} & (Constant) & 246.227 & 17.789 & & 13.841 & .000 & & \\
\hline & URB & 26.052 & 10.169 & .078 & 2.562 & .012 & .997 & 1.003 \\
\hline & LN1 & 161.812 & 12.920 & .457 & 12.524 & .000 & .702 & 1.424 \\
\hline & LN2 & 64.773 & 12.492 & .189 & 5.185 & .000 & .703 & 1.422 \\
\hline & Ratio2 & 844.414 & 37.108 & .808 & 22.756 & .000 & .741 & 1.349 \\
\hline & Vol & -679 & .024 & -.986 & -27.918 & .000 & .749 & 1.336 \\
\hline
\end{tabular}

a. Dependent Variable: Cap

Table 25 - Validation Results for Permissive-Only Capacity Models for Non-Zero Permissive Capacities

\begin{tabular}{cccccc} 
Dataset & N & MSPE & MAE & MAPE & Mean Bias \\
\hline Build & 80 & 1903.0 & 34.47 & $12.95 \%$ & 0.00 \\
Validation & 21 & 1892.4 & 30.77 & $11.15 \%$ & -15.62
\end{tabular}

\subsubsection{Left-Turn Conflict Models}

Two left-turn conflict prediction models were created using SPSS, corresponding to nonzero permissive capacity cases of PPLT and non-zero permissive capacity cases of permissiveonly phasing. The zero-permissive capacity cases for permissive-only and PPLT phasing modes were not modeled, as conflicts for these cases were assumed to be zero. For both of these models, model fits were not as good as the capacity models, but still produced acceptable results. Additionally, regression assumptions were met, and validation was performed.

\subsubsection{PPLT Left-Turn Conflict Model for Non-Zero Permissive Capacities}

Table 26 shows the ANOVA for PPLT conflicts when the permitted capacity is greater than zero. The discrete variables for the protected green ratio, along with the continuous 
variables for subject street green ratio, volume, and speed produced statistically significant effects on conflicts.

Table 26 - ANOVA Results for PPLT Conflict Model for Non-Zero Permissive Capacities

\begin{tabular}{|c|c|c|c|c|c|}
\hline \multicolumn{6}{|c|}{ Dependent Variable: PCon } \\
\hline Source & $\begin{array}{l}\text { Type III Sum } \\
\text { of Squares }\end{array}$ & df & Mean Square & $\mathrm{F}$ & Sig. \\
\hline Corrected Model & $837.897^{\mathrm{a}}$ & 27 & 31.033 & 13.532 & .000 \\
\hline Intercept & 168.977 & 1 & 168.977 & 73.681 & .000 \\
\hline Cycle & .695 & 1 & .695 & .303 & .583 \\
\hline Ratio & 395.012 & 1 & 395.012 & 172.241 & .000 \\
\hline Vol & 40.693 & 1 & 40.693 & 17.744 & .000 \\
\hline Speed & 102.575 & 1 & 102.575 & 44.727 & .000 \\
\hline AreaTyp & 2.569 & 1 & 2.569 & 1.120 & .291 \\
\hline OppLn & 3.778 & 2 & 1.889 & .824 & .440 \\
\hline ProtRatio & 416.361 & 3 & 138.787 & 60.517 & .000 \\
\hline AreaTyp * OppLn & .974 & 2 & .487 & .212 & .809 \\
\hline AreaTyp * ProtRatio & 5.568 & 3 & 1.856 & .809 & .490 \\
\hline OppLn * ProtRatio & 2.733 & 6 & .456 & .199 & .977 \\
\hline $\begin{array}{l}\text { AreaTyp * OppLn * } \\
\text { ProtRatio }\end{array}$ & 3.705 & 6 & .617 & .269 & .951 \\
\hline Error & 460.966 & 201 & 2.293 & & \\
\hline Total & 4282.089 & 229 & & & \\
\hline Corrected Total & 1298.864 & 228 & & & \\
\hline
\end{tabular}

a. R Squared $=.645$ (Adjusted R Squared $=.597$ )

Equation 12 shows the conflict prediction model that was produced from stepwise regression. The final parameters in the model were the dummy variables for the different levels of PPLT and continuous variables for subject street green ratio, opposing volume (with a quadratic transformation), and average opposing speed. The coefficients of the model are shown in Table 27, and the model had an adjusted $\mathrm{R}^{2}$ value of 0.709 . Additionally, the validation results are listed in Table 28. While validation results were relatively worse for conflict prediction 
models than capacity models, the large percentage errors are attributable to the small magnitudes of conflicts. Additionally, the build and validation datasets closely matched in terms of the statistics calculated, indicating the model fit each dataset similarly.

$$
\begin{aligned}
\operatorname{con}_{100}=- & 15.41+(3.939 * P P 10)+(2.110 * P P 15) \\
& +(0.9920 * P P 20)+\left(12.96 * \frac{G}{C}\right)+(0.01653 * q) \\
& -\left(1.751 \times 10^{-5} * q^{2}\right)+(0.1194 * S)
\end{aligned}
$$

where

\begin{tabular}{|c|c|c|c|c|c|c|c|c|}
\hline \multicolumn{9}{|c|}{ Coefficients $^{a}$} \\
\hline \multirow[b]{2}{*}{ Mode } & & \multicolumn{2}{|c|}{$\begin{array}{l}\text { Unstandardized } \\
\text { Coefficients }\end{array}$} & \multirow{2}{*}{$\begin{array}{c}\text { Standardized } \\
\text { Coefficients } \\
\text { Beta }\end{array}$} & \multirow[b]{2}{*}{$\mathrm{t}$} & \multirow[b]{2}{*}{ Sig. } & \multicolumn{2}{|c|}{ Collinearity Statistics } \\
\hline & & $\mathrm{B}$ & Std. Error & & & & Tolerance & VIF \\
\hline \multirow[t]{8}{*}{1} & (Constant) & -15.414 & 1.139 & & -13.528 & .000 & & \\
\hline & PP10 & 3.939 & .286 & .830 & 13.786 & .000 & .440 & 2.271 \\
\hline & PP15 & 2.110 & .293 & .416 & 7.210 & .000 & .481 & 2.080 \\
\hline & PP20 & .992 & .301 & .178 & 3.299 & .001 & .550 & 1.819 \\
\hline & Ratio & 12.958 & .883 & .661 & 14.670 & .000 & .786 & 1.273 \\
\hline & Vol & .017 & .002 & 1.478 & 6.719 & .000 & .033 & 30.290 \\
\hline & Vol2 & $-1.751 \mathrm{E}-5$ & .000 & -1.723 & -7.753 & .000 & .032 & 30.914 \\
\hline & Speed & .119 & .016 & .307 & 7.609 & .000 & .980 & 1.021 \\
\hline
\end{tabular}

$$
\begin{gathered}
\operatorname{con}_{100}=\text { number of conflicts per } 100 \text { left-turning vehicles, and } \\
S=\quad \text { average opposing speed. }
\end{gathered}
$$

Table 27 - Regression Parameters for PPLT Conflict Model for Non-Zero Permissive Capacities

a. Dependent Variable: PCon

Table 28 - Validation Results for PPLT Conflict Models for Non-Zero Permissive Capacities

\begin{tabular}{cccccc} 
Dataset & N & MSPE & MAE & MAPE & Mean Bias \\
\hline Build & 183 & 1.5 & 0.94 & $26.31 \%$ & -0.13 \\
Validation & 46 & 2.1 & 1.08 & $28.98 \%$ & -0.24
\end{tabular}




\subsubsection{Permissive-Only Left-Turn Conflict Model for Non-Zero Permissive Capacities}

Table 29 shows the ANOVA results for conflicts for permitted phasing with a non-zero permitted capacity. The fixed variable for opposing lanes and continuous variables for cycle length, green ratio, opposing volume, and speed were found to create statistically significant effects on left turn conflicts.

Table 29 - ANOVA Results for Permissive-Only Conflict Model for Non-Zero Permissive Capacities

\begin{tabular}{|c|c|c|c|c|c|}
\hline \multicolumn{6}{|c|}{ Tests of Between-Subjects Effects } \\
\hline Source & $\begin{array}{c}\text { Type III Sum } \\
\text { of Squares }\end{array}$ & df & Mean Square & $\mathrm{F}$ & Sig. \\
\hline Corrected Model & $1376.861^{\mathrm{a}}$ & 9 & 152.985 & 17.601 & .000 \\
\hline Intercept & 360.344 & 1 & 360.344 & 41.459 & .000 \\
\hline Cycle & 141.174 & 1 & 141.174 & 16.243 & .000 \\
\hline Ratio & 307.754 & 1 & 307.754 & 35.408 & .000 \\
\hline Vol & 166.937 & 1 & 166.937 & 19.207 & .000 \\
\hline Speed & 241.720 & 1 & 241.720 & 27.811 & .000 \\
\hline AreaTyp & 8.713 & 1 & 8.713 & 1.002 & .319 \\
\hline OppLn & 70.377 & 2 & 35.188 & 4.049 & .021 \\
\hline AreaTyp * OppLn & 7.519 & 2 & 3.759 & .433 & .650 \\
\hline Error & 790.935 & 91 & 8.692 & & \\
\hline Total & 11984.843 & 101 & & & \\
\hline Corrected Total & 2167.796 & 100 & & & \\
\hline
\end{tabular}

a. $\mathrm{R}$ Squared $=.635$ (Adjusted R Squared $=.599$ )

Next, stepwise regression was used to create a model to predict conflicts when capacities are not zero. The dummy variable for the two opposing lane configurations was identified as the only dummy variable that was statistically significant in the models, but it was not used in the final model, as it would group one and three opposing lane configurations into one prediction, which was not logical. The final conflicts per 100 left-turning vehicles model for permissiveonly left-turn mode for non-zero permissive capacities had only continuous variables as 
significant parameters, which were found to be cycle length, subject street green ratio, the inverse of the opposing volume, and the average speed of the opposing direction. This model had a 0.599 adjusted $\mathrm{R}^{2}$ value, and the equation for the model is shown below in Equation 13. Additionally, the coefficients for the parameters used in the model are shown in Table 30, and the results from model validation are shown in Table 31. Again, large percent errors can be attributed to the small magnitudes of conflicts, as well as the model fit determined by the adjusted $\mathrm{R}^{2}$ value. Additionally, the validation dataset appeared to have fit the model better than the build dataset, with the exception of the mean bias statistic, indicating an inconsistent performance of the model.

$\operatorname{con}_{100}=-12.10+(0.2685 * C)+\left(14.12 * \frac{G}{C}\right)-\left(\frac{1884}{q}\right)+(0.2962 * S)$

Table 30 - Regression Parameters for Permissive-Only Conflict Model for Non-Zero Permissive Capacities

\begin{tabular}{|c|c|c|c|c|c|c|c|c|}
\hline \multicolumn{9}{|c|}{ Coefficients $^{a}$} \\
\hline \multirow[b]{2}{*}{ Model } & & \multicolumn{2}{|c|}{ Unstandardized Coefficients } & \multirow{2}{*}{$\begin{array}{c}\text { Standardized } \\
\text { Coefficients } \\
\text { Beta }\end{array}$} & \multirow[b]{2}{*}{$t$} & \multirow[b]{2}{*}{ Sig. } & \multicolumn{2}{|c|}{ Collinearity Statistics } \\
\hline & & B & Std. Error & & & & Tolerance & VIF \\
\hline \multirow[t]{5}{*}{1} & (Constant) & -12.102 & 3.444 & & -3.514 & .001 & & \\
\hline & Cycle & .027 & .007 & .273 & 3.661 & .000 & .986 & 1.014 \\
\hline & Ratio & 14.121 & 2.782 & .399 & 5.075 & .000 & .887 & 1.128 \\
\hline & Vollnv & -1884.240 & 356.797 & -.420 & -5.281 & .000 & .867 & 1.153 \\
\hline & Speed & .296 & .060 & .375 & 4.968 & .000 & .964 & 1.038 \\
\hline
\end{tabular}

a. Dependent Variable: PCon

Table 31 - Validation Results for Permissive-Only Conflict Models for Non-Zero Permissive Capacities

\begin{tabular}{cccccc} 
Dataset & N & MSPE & MAE & MAPE & Mean Bias \\
\hline Build & 74 & 8.6 & 2.34 & $24.97 \%$ & 0.00 \\
Validation & 27 & 4.3 & 1.71 & $15.37 \%$ & -0.23
\end{tabular}




\subsubsection{Summary of Findings}

One of the most impactful findings of this phase of the research was the set of thresholds to determine when permissive capacities cannot be provided by either PPLT or permissive-only left-turn phasing, based on protected green ratio, subject street green ratio, and opposing volume (flow rate), found in Table 15 and Table 16. The reason for this is that the thresholds establish circumstances under which these phasing modes should not be considered, and protected-only left-turns should be the selected control mode if the left-turn demand cannot be met purely from sneaker capacities for permissive-only phasing. Since the simulation models imitated safe driving behaviors, if turns could not be made during the permissive phase, real-world drivers would have a higher risk of causing a crash if any permitted phasing was provided. This would occur because some drivers would likely get impatient, believing they should be able to execute their turn during the permissive portion of the phase, and cross traffic during inadequate gaps.

Three capacity and two conflict models were created in this portion of the study, representing capacity models for non-zero permissive capacities for PPLT, zero-permissive capacities for PPLT, and non-zero permissive capacities for permissive-only phasing mode, as well as conflict models for non-zero permissive capacities for both PPLT and permissive-only. Coefficients were found to be logical in magnitude and sign for each of the models, and parameters excluded from models were found to be appropriate. Additionally, all regression assumptions were checked and found to be unviolated.

The three remaining phasing mode predictions were derived from assumptions, rather than modeled using regression. For scenarios over the thresholds for permissive-only mode developed, a capacity consisting of only sneakers is assumed (two sneakers per cycle), as these were defined as zero-permissive capacity cases in determining the thresholds. For cases with 
zero-permissive capacities for PPLT and permissive-only phasing, it can also be assumed that zero conflicts will occur. Though these cases were determined to have zero conflicts from the simulations, this does not indicate that these situations are safe for left-turning drivers. As mentioned earlier in this section, cases with zero-permissive capacity provide danger due to driver habits, such as impatience or misjudging gaps in opposing vehicle; therefore, traffic engineers should carefully evaluate these cases to determine if permissive phasing of any type is appropriate. 


\section{CHAPTER 5: RISK ASSESSMENT MODEL FOR TIME-OF-DAY SAFETY} ANALYSIS

Number of conflicts, as derived by the prediction models developed in the previous chapter of this document, are not a commonly-used safety metric by practitioners; therefore, a model to assess crash risk based on conflicts was created. Additionally, a time-of-day safety analysis measure is not currently available to engineers, thus a risk assessment model based on varying conflicts should be developed. Six intersections from the Southwest region of Virginia were selected to build the risk assessment model, based on availability of complete signal timing and hourly volume data. The number of conflicts, along with SPF-predicted hourly crash rates, were determined using the prediction models created by this study and Virginia-specific SPFs. A model was then developed to relate predicted crash frequencies to conflicts using the data from the intersections. Finally, the model was validated with data that was initially set aside from the model construction dataset.

\subsection{METHODOLOGY}

\subsubsection{Data Compilation}

The model for assessing crash risk based on number of predicted conflicts was based on data from six intersection sites in the Southwest region of Virginia. These intersections were identified as analysis sites since hourly turning movement counts and signal timing plans were readily available, as provided by VDOT traffic engineers. Available data included the workbooks used for the signal studies that were conducted by VDOT, turning movement counts, Synchro file outputs, among other files. Information needed to predict conflicts and crashes needed to be extracted from these files to gather data for model development. Because of a lack 
of full-day timing plans, PM peak hour timing plans for each of the intersections were assumed to represent average signal parameters needed for the conflict models throughout the analysis day. Signal timing information needed for the conflict prediction models such as green ratios and cycle lengths were extracted from the Synchro output files for the proposed timing plans for each intersection approach. Left-turn phasing modes for each approach were then determined from the signal study workbooks. Site characteristics, such as speed limits and lane geometries were also obtained from the files provided. Finally, hourly turning movement counts for all vehicles over a 24 -hour period were copied into a spreadsheet. Once information pertinent to each intersection and approach was compiled into one workbook, calculations could be performed to predict conflicts and crashes. A summary of the intersections can be found below in Table 32, and specific parameters for the approaches of each intersection are listed in Table 33, with asterisks indicating that the value was adjusted to fit the model restrictions (i.e. some average speeds were adjusted from $25 \mathrm{mph}$ to $35 \mathrm{mph}$ ).

Table 32 - Intersection Characteristics

\begin{tabular}{cccccc} 
Site & Intersection & Locality & $\begin{array}{c}\text { \# of } \\
\text { Legs }\end{array}$ & $\begin{array}{c}\text { Approach } \\
\text { Phasing Modes }\end{array}$ & $\begin{array}{c}\text { Average } \\
\text { Cycle } \\
\text { Length }\end{array}$ \\
\hline A & $\begin{array}{c}\text { Main Street and Industrial } \\
\text { Park Drive }\end{array}$ & $\begin{array}{c}\text { Montgomery } \\
\text { County }\end{array}$ & 4 & 4 PPLT-FYA & $107.5 \mathrm{~s}$ \\
\hline B & $\begin{array}{c}\text { Main Street and } \\
\text { Professional Park Drive }\end{array}$ & $\begin{array}{c}\text { Montgomery } \\
\text { County }\end{array}$ & 4 & 4 PPLT-FYA & $88 \mathrm{~s}$ \\
\hline C & Route 220 and Route 1290 & $\begin{array}{c}\text { Roanoke } \\
\text { County }\end{array}$ & 4 & $\begin{array}{c}\text { 2 PPLT-FYA, } 2 \\
\text { PO }\end{array}$ & $153.1 \mathrm{~s}$ \\
\hline D & Route 220 and Route 862 & $\begin{array}{c}\text { Roanoke } \\
\text { County }\end{array}$ & 4 & $\begin{array}{c}\text { 2 PPLT-FYA, } \\
\text { PO }\end{array}$ & $162.8 \mathrm{~s}$ \\
\hline E & Route 221 and Route 687 & $\begin{array}{c}\text { Roanoke } \\
\text { County }\end{array}$ & 4 & $\begin{array}{c}\text { 3 PPLT-FYA, 1 } \\
\text { PO }\end{array}$ & $131.9 \mathrm{~s}$ \\
\hline F & Route 220 and Route 789 & $\begin{array}{c}\text { Roanoke } \\
\text { County }\end{array}$ & 3 & 1 PPLT-FYA & $155.7 \mathrm{~s}$
\end{tabular}

All intersections are of Urban area type and are located in the Western Operational region 
Table 33 - Intersection Approach-Level Characteristics

\begin{tabular}{|c|c|c|c|c|c|}
\hline Site & Approach & $\begin{array}{c}\text { Protected } \\
\text { Ratio }\end{array}$ & $\begin{array}{c}\text { Green } \\
\text { Ratio }\end{array}$ & $\begin{array}{c}\text { Opposing } \\
\text { Lanes }\end{array}$ & Speed \\
\hline \multirow{4}{*}{ A } & Northbound & 0.147 & \multirow{2}{*}{0.612} & \multirow{2}{*}{2} & \multirow{2}{*}{45} \\
\hline & Southbound & 0.099 & & & \\
\hline & Eastbound & 0.127 & \multirow{2}{*}{0.388} & \multirow{2}{*}{1} & \multirow{2}{*}{$35^{*}$} \\
\hline & Westbound & 0.145 & & & \\
\hline \multirow{4}{*}{ B } & Northbound & 0.130 & \multirow{2}{*}{0.698} & \multirow{2}{*}{2} & \multirow{2}{*}{45} \\
\hline & Southbound & 0.116 & & & \\
\hline & Eastbound & 0.099 & \multirow{2}{*}{0.302} & \multirow{2}{*}{1} & \multirow{2}{*}{$35^{*}$} \\
\hline & Westbound & 0.153 & & & \\
\hline \multirow{4}{*}{ C } & Northbound & $0.075^{*}$ & \multirow{2}{*}{0.736} & \multirow{2}{*}{2} & \multirow{2}{*}{45} \\
\hline & Southbound & 0.115 & & & \\
\hline & Eastbound & - & \multirow{2}{*}{-} & \multirow{2}{*}{-} & \multirow{2}{*}{-} \\
\hline & Westbound & - & & & \\
\hline \multirow{4}{*}{ D } & Northbound & 0.100 & \multirow{2}{*}{0.790} & \multirow{2}{*}{2} & \multirow{2}{*}{45} \\
\hline & Southbound & 0.090 & & & \\
\hline & Eastbound & - & \multirow{2}{*}{-} & \multirow{2}{*}{ - } & \multirow{2}{*}{-} \\
\hline & Westbound & - & & & \\
\hline \multirow{4}{*}{ E } & Northbound & 0.160 & \multirow{2}{*}{0.510} & \multirow{2}{*}{2} & \multirow{2}{*}{45} \\
\hline & Southbound & 0.130 & & & \\
\hline & Eastbound & 0.127 & \multirow{2}{*}{0.490} & \multirow{2}{*}{1} & $35 *$ \\
\hline & Westbound & - & & & 30 \\
\hline & Northbound & - & $08 *$ & 2 & 45 \\
\hline$F$ & Southbound & 0.110 & & & \\
\hline & Eastbound & - & & - & 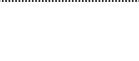 \\
\hline & Westbound & - & & - & - \\
\hline
\end{tabular}

\subsubsection{Conflict Calculations}

Using the models developed in Chapter 4, predicted conflicts were calculated for each hour of the six selected intersections on a left-turn approach level. The conflict prediction model that was used for each hour and each approach was determined by first evaluating whether or not 
permissive capacity could be provided for the particular phasing mode. If it was determined that no permissive capacity could be provided, or the approach operated in protected-only mode, zero conflicts per 100 left-turning vehicles were assumed for that hour, otherwise the appropriate prediction model was used, with negative predictions being assumed as zero conflicts. Also, if for a particular hour the opposing volumes were outside of the range of the 200-1,200 veh/hr/ln used in developing the conflict and capacity models, that hour was not used in further development of the models. Predictions of conflicts per 100 left-turning vehicles were then multiplied by the left-turn demands for each hour, divided by 100 , to find the number of conflicts for that hour. Finally, conflicts were summed across each left-turn approach for the intersectionlevel conflicts to be used in the crash prediction model development.

\subsubsection{Crash Frequency Calculations}

Average annual crash frequencies were calculated on an hourly basis for each intersection to compare with the predicted conflicts. Although a model for predicting these frequencies on an hourly basis does not currently exist, these were estimated using the Virginia-specific SPFs used in the CMF development section of this chapter. To do this, turning movement counts were summed across streets and multiplied by 24 to estimate an equivalent daily volume for each hour. Next, annual crashes were predicted using turning movement counts for the entire 24-hour period, representing estimated AADT values, and were used in calculating crash frequencies for a "true" prediction of daily crashes. Each of the previously-calculated hourly crash frequencies were then normalized by multiplying them by the sum of the hourly crash frequencies over the 24-hours, divided by the true crash frequency for the day. In doing so, the sum of the normalized crash counts across the 24-hours was equal to the annual crash count based on the daily traffic. 
These counts represented the number of crashes that would be expected in that hour if the characteristics of that hour were the same throughout the course of a year. While this is not a perfect estimate of traffic safety, it does provide a scalable metric that can be used to assess relative safety based on volume.

Similar to the approach used in developing CMFs, base conditions were adjusted for by calculating base CMFs for the site-specific characteristics defined in the HSM, including leftturn phasing. Once the SPF-predicted crash rates were calculated for each hour, the base CMFs were applied. Additionally, percentages of angle crashes found in the CMF development section of this chapter were multiplied by the predictions to determine the predicted number of angle crashes, the target crash type to be compared to left-turn conflicts. Values of $43 \%$ for $3-\operatorname{leg}$ intersections and 53\% angle crashes for 4-leg intersections were assumed.

\subsubsection{Model Development}

A model to predict the number of yearly crashes for a particular hour at an intersection based on that hour's conflict predictions was developed in SPSS, using a similar process as the development of the conflict and capacity models described in Chapter 4. Similar to the process taken to develop the capacity and conflict models, three main steps were taken: transformations of the conflicts variable, model selection, and model validation. Data used in the models consisted of each hour's conflicts and estimated crashes for all sites that conflicts where able to be calculated for each approach (76 total hours) as the independent and dependent variables, respectively. Similar to the previously developed models, $75 \%$ of data was used to build the model and the rest of the data was used as hold-back data for validation. 


\subsubsection{Linear Regression and Model Selection}

Using the curve fit tool in SPSS, several transformations on the independent variable, number of conflicts in an hour, were tested for their fit on the crash prediction data. $\mathrm{R}^{2}$ values and plots were compared across the different model forms. A similar process as was taken with the conflict and capacity models was used to compare the linear regression models resulting from the different model forms, although only one independent variable was used. Once each of the linear regression models was created, adjusted $\mathrm{R}^{2}$ values were compared against one another, P-P plots were checked for linearity, standardized residuals were checked for a normal distribution, and parameter coefficients were checked to make sure they were logical in sign and order of magnitude. Additionally, for each model, the residuals for the independent variable was plotted against the variable to check for linearity, homoscedasticity, and independence of errors. Of all the models, the optimal model was selected as the model that fit the data best while conforming to the regression assumptions, though in some cases the increase in model fit over another model outweighed the marginal decrease in regression assumption conformity. An additional check was also completed to determine the difference in SPF-predicted crashes vs the model-predicted crashes aggregated within the sites. Also, the model was compared with a previously-computed model developed in the SSAM Final Report (30).

\subsubsection{Model Validation}

Once the most optimal model form was selected from the linear regression stage, a final model was created using $75 \%$ of the original dataset. The model was then compared against the hold-back dataset to ensure validity of the prediction model. Using the number of conflicts, predictions for the crash rates were calculated using the model form for all of the 76 data points. 
Mean square prediction error, mean absolute error, mean absolute percent error, and mean bias were calculated for the validation and build datasets so that they could be compared. Once it was confirmed that both sets of data produced similar results, the final model was complete.

\subsection{RESULTS}

In determining the best model for predicting annual crash frequencies based on the number of conflicts, a final linear model was found, with the following equation, Equation 14. Outputs from SPSS for the model parameter and constant is shown in Table 34, and the results from model validation, which includes statistics from the build and validation datasets, are shown in Table 35. An adjusted $\mathrm{R}^{2}$ value for this model was determined to be 0.421 , and errors calculated were higher than $34 \%$ for the build and validation data. Model validation provided similar results between the two datasets, with the exception of a large difference in MAPE, which is likely due to the suboptimal model fit. It is also important to note that the prediction of this model results in an annual crash rate that would result from a constant level of conflicts over the course of an entire year for the corresponding hour of the day; therefore, these results should be used mostly as a relative risk measure rather than as an estimate of annual crash rates.

annual crashes per hour

$=0.0638+(0.00858 *$ number of conflicts per hour $)$ 
Table 34 - Regression Parameters for Crash Model

\begin{tabular}{|c|c|c|c|c|c|c|c|c|}
\hline \multicolumn{9}{|c|}{ Coefficients $^{a}$} \\
\hline \multirow[b]{2}{*}{ Model } & & \multicolumn{2}{|c|}{ Unstandardized Coefficients } & \multirow{2}{*}{$\begin{array}{c}\text { Standardized } \\
\text { Coefficients } \\
\text { Beta } \\
\end{array}$} & \multirow[b]{2}{*}{$\mathrm{t}$} & \multirow[b]{2}{*}{ Sig. } & \multicolumn{2}{|c|}{ Collinearity Statistics } \\
\hline & & B & Std. Error & & & & Tolerance & VIF \\
\hline \multirow[t]{2}{*}{1} & (Constant) & .064 & .010 & & 6.216 & .000 & & \\
\hline & Con & .009 & .001 & .657 & 6.464 & .000 & 1.000 & 1.000 \\
\hline
\end{tabular}

a. Dependent Variable: SPF

Table 35 - Validation Results for Crash Model

\begin{tabular}{cccccc} 
Dataset & N & MSPE & MAE & MAPE & Mean Bias \\
\hline Build & 57 & 0.002 & 0.039 & $34.10 \%$ & 0.00 \\
Validation & 19 & 0.002 & 0.045 & $40.12 \%$ & 0.00
\end{tabular}

In addition to the standard model validation process, the crash predictions produced by the risk assessment model were compared across the sites used to build the model, shown in Table 36. As with the mean bias shown above, the percent difference across all sites was one percent, meaning the overpredictions for some sites mostly matched the underpredictions for others. The site with the greatest error was Site F, which could be explained by the fact that it was the only 3-leg site. Additionally, Sites A through D performed similarly, with Site E proving to be different. This difference may be explained by the number of approaches with PPLT-FYA, as this site had an odd number of legs treated. From these results, four-leg intersections with two legs treated performed the best with the model created and the four-leg intersections with three legs treated were similar to absolute percent errors for the entire dataset, so they had acceptable performance. The three-leg intersections using this model performed poorly; therefore, the model is not as reliable for these intersection configurations. However, all crash predictions were within $1 \mathrm{crash} / \mathrm{yr}$ of what was predicted by the SPF. 
Table 36 - Crash Predictions Across Separate Sites

\begin{tabular}{cccc}
\multicolumn{5}{c}{ Predicted Crashes } \\
Site & SPF & Model & \% Difference \\
\hline A & 0.32 & 0.23 & $-29 \%$ \\
B & 0.97 & 1.23 & $27 \%$ \\
C & 2.42 & 2.53 & $4 \%$ \\
D & 2.51 & 2.09 & $-17 \%$ \\
E & 1.36 & 0.82 & $-40 \%$ \\
F & 1.14 & 1.92 & $68 \%$ \\
\hline & 8.72 & 8.81 & $1 \%$
\end{tabular}

The model developed in this study was compared to the crash prediction model developed in the SSAM Final Report (30), shown by Equation 15. Predictions using the conflict data in this portion of the study were made using this model and divided by 24 to compare with the prediction model developed in this study. Results from these calculations were compared across the individual sites and on an aggregate level, as with the calculations from the studydeveloped model. These results are shown below in Table 37, and indicate poor performance when compared to the study-developed model.

$$
\text { annual crashes }=0.119^{\text {number } \text { of } \text { conflicts } * 1.419}
$$


Table 37 - SSAM Model Crash Predictions Across Separate Sites

\begin{tabular}{cccc}
\multicolumn{5}{c}{ Predicted Crashes } \\
Site & SPF & SSAM Model & \% Difference \\
\hline A & 0.32 & 0.03 & $-92 \%$ \\
B & 0.97 & 0.32 & $-67 \%$ \\
C & 2.42 & 2.38 & $-2 \%$ \\
D & 2.51 & 1.43 & $-43 \%$ \\
E & 1.36 & 0.16 & $-88 \%$ \\
F & 1.14 & 1.42 & $24 \%$ \\
\hline & 8.72 & 5.72 & $-34 \%$
\end{tabular}

\subsubsection{Summary of Findings}

Developed with SPF-predicted angle crashes for six Virginia intersections with adjustments for base CMFs and percentage of angle crashes, a risk assessment model based on number of predicted conflicts calculated from the models in Chapter 4, was created. In creating this model, different parameters' effects on angle crashes can be quantified than was available previously, such as signal timing, opposing volume, and site characteristic parameters. This enables time-of-day evaluation of left-turn phasing previously unavailable. Additionally, this model could be incorporated into the spreadsheet tool for practitioners developed in this research, described in Chapter 6.

Although a model already existed for predicting annual crash frequencies based on number of conflicts, developed in the SSAM Final Report (30), the existing model did not perform nearly as well as the model developed in this study for Virginia intersection data. This was evident when comparing the two prediction models - the SSAM model for crash rates predicted 34 percent of the SPF-predicted crashes (the ground truth for the purpose of this study), when summed across all hours of the day, most likely since the SSAM model is only able to predict the number of crashes from all conflict types. Because of this, the model created by this 
research should be used for Virginia intersections and for left-turn conflicts, as was the intent. Also, the SSAM model uses a power model form where the conflicts are raised to the 1.419 power and then multiplied by 0.119 . Because of this, if zero conflicts are predicted, zero angle crashes are predicted to occur in a given year, which is not realistic, as crashes are random event and can be caused by human error (i.e. red light running). This is avoided in the model developed in this study by having a linear model form, with an average number of annual crashes as a constant, minimum value. This model form appeared to fit the SPF-predicted crashes better, as there were several cases with zero predicted conflicts and non-zero SPF-predicted crashes. 


\section{CHAPTER 6: SPREADSHEET TOOL FOR PRACTITIONERS}

With models to predict left-turn capacities, conflicts, and crash risk for permissive-only and PPLT left-turn phasing control modes having been developed, a spreadsheet tool was created to assist traffic engineers in using the models for intersections they wish to analyze. The tool requires users to input static input parameters as well as timing variables and volume counts for each analysis hour. Then, the spreadsheet calculates the left-turn capacities, volume to capacity ratios, number of conflicts per 100 left-turning vehicles, and crash risk using the appropriate models, assumptions, and calculations determined in previous chapters of this research. Finally, capacities and crash risk are plotted for each analysis hour in separate graphs for a visual representation of the operational and safety variations across a typical day. This spreadsheet tool is intended to aid field engineers in their left-turn mode decisions, rather than attempting to define strict guidelines.

\subsection{CONSTRUCTION OF SPREADSHEET}

\subsubsection{Inputs}

For the tool to work properly, input parameters and traffic counts must be specified by the engineer for the intersection they are analyzing. These parameters were the predictor variables required for the prediction models developed in Chapters 4 and 5, and are used for calculations in the spreadsheet. Cells in the spreadsheet that require information from the engineer were colored green to indicate inputs. As some parameters do not change throughout the course of a day and some are variable across each hour, two sets of parameters were required: constant parameters and hourly inputs, outlined below. 


\subsubsection{Constant Parameters}

Area type, number of opposing lanes, and speed limit of opposing lanes were the three constant parameters required for the spreadsheet, as these are site characteristics and do not vary by time-of-day. For these parameters, a table was provided at the top-left corner of the spreadsheet, with separate cells for the individual parameters. The input cells were formatted using the "Data Validation" tool in Microsoft Excel to ensure that valid inputs are given, as defined by the ranges for the models. For area type, a dropdown menu with options of "Urban" and "Rural" was provided. Number of lanes was also given a dropdown menu, with options of one, two, or three opposing lanes. Finally, the speed limit of opposing lanes (which was used as the average speed of opposing lanes predictor variable in the model calculations) required the value to be any value between 35 and $55 \mathrm{mph}$.

\subsubsection{Hourly Inputs}

For each hour of the analysis period, hourly inputs must be specified to achieve results from the spreadsheet tool, as these were used in model predictions and calculations. Cycle length, protected green ratio, subject street green ratio, yellow plus all red duration, opposing volumes, and left-turn volumes were indicated as parameters that can vary throughout the course of a day, as they pertain to timing parameters and traffic volumes. Similar to the constants, parameters associated with signal timing were restricted to ranges that corresponded to the predictor variable ranges for the capacity and conflict models; cycle length could be any value between 80 s and 240s, protected green ratios could be between 0.075 and 0.274 (as these are rounded to the nearest 0.05 in the calculations), subject street green ratios could be any value between 0.3 and 0.8 , and the yellow plus all red time parameter could be any value between 0 
and the cycle length multiplied by the protected green ratio. Restrictions were not set for the opposing and left-turn volume inputs, as these are field-reported values that should not be altered, though the spreadsheet could not make predictions if opposing volumes were outside of the acceptable range. Additionally, it should be noted that input volumes were required in units of vehicles per hour, so that the engineer could insert data directly from their turning movement counts without having to make any calculations.

\subsubsection{Predictions}

\subsubsection{Left-Turn Capacity}

Capacities for the left-turn movements were calculated for each analysis hour for three different phasing modes: PPLT, permissive-only, and protected-only. For PPLT and permissiveonly cases, appropriate model equations were used to complete these calculations. If for a particular hour the opposing volume was outside of the 200 to $1,200 \mathrm{veh} / \mathrm{hr} / \mathrm{ln}$ range established in model development, the capacities for that hour for PPLT and permissive-only modes were left blank. Protected-only capacities were calculated using Equation 16, derived from the HCM, with the 1.05 term representing a left-turn factor for saturation flow rate.

$$
c_{\text {protected-only }}=\left(\frac{G}{C}_{\text {prot }}-\frac{l}{C}\right) \frac{s}{1.05}
$$

where

$$
\begin{array}{cl}
\frac{G}{C_{\text {prot }}}= & \text { protected green ratio (decimal), } \\
l= & \text { loss time (yellow and all red time }+2 \mathrm{~s})(\mathrm{s}), \\
C= & \text { cycle length }(\mathrm{s}), \text { and } \\
s= & \text { ideal saturation flow rate, } 1,900 \text { for urban and } 1,750 \text { for rural }(\mathrm{veh} / \mathrm{hr} / \mathrm{ln}) .
\end{array}
$$




\subsubsection{Volume-to-Capacity Ratio (v/c)}

For easy identification of the percentage of capacity being used by the demand left-turn volume for each hour and each phasing mode, volume-to-capacity ratios (v/c) were calculated. This was done by simply dividing the demand left-turn volume by the capacities produced in the previous section of this document. In cases where PPLT and permissive-only capacities were not calculated due to the opposing volumes being outside of the appropriate range, the $\mathrm{v} / \mathrm{c}$ for that hour was left blank for these two modes. Additionally, conditional formatting was set in the Excel document such that $\mathrm{v} / \mathrm{c}$ values greater than 1.0 were automatically highlighted in red to identify cases where the phasing mode could not provide enough capacity to process all vehicles in that hour.

\subsubsection{Conflicts per 100 Left-Turning Vehicles}

Using the models developed by this study, the number of conflicts per 100 left-turning vehicles was calculated for each hour for the PPLT and permissive-only phasing control modes. As the study did not simulate protected-only scenarios and conflict prediction models are not currently available for this mode, predictions were not made for protected-only phasing. Using these values, the number of conflicts in an hour can be determined by multiplying the prediction by the left-turn demand volume and dividing by 100 . These values are then used to determine average annual angle crash frequencies for each hour, described in the following section. As with the capacity and v/c calculations, hours with opposing volumes outside of the modeled range were left blank for the conflicts per 100 left-turning vehicles calculations. 


\subsubsection{Crash Risk}

The average number of annual angle crashes was calculated for each set of conditions to illustrate the relative risk of permissive-only and PPLT modes over the course of a typical day, with changing conditions. This was done by using the crash risk assessment model, described in the previous chapter, Chapter 5, with the number of conflicts generated in the previous step of the spreadsheet tool. Again, hours with opposing volumes less than $200 \mathrm{veh} / \mathrm{hr} / \mathrm{ln}$ or greater than $1,200 \mathrm{veh} / \mathrm{hr} / \mathrm{ln}$ were left blank for these predictions. Additionally, it should be noted that these predictions represent the number of crashes predicted for a year if the same conditions existed for the entire year; therefore, the relative magnitude of these predictions when compared across control mode and hour are more important than the values themselves. Because of this, the outputs from this model should be used in evaluating relative risk, rather than predicting crashes.

\subsubsection{Graphs}

The final step in developing the spreadsheet tool was to create scatterplots that illustrated how left-turn capacities and angle crash risk varied throughout the analysis period. In both graphs, the $\mathrm{x}$-axis represented the hour of the day, from 0 to 23 , representing midnight to $11 \mathrm{pm}$. The $y$-axis for the capacity graph represented the predicted left-turn capacity, in vehicles per hour, and for the crash frequency graph represented the average annual angle crash frequency, in

crashes per year. In the capacity plot, predicted capacities for each of the three left-turn phasing control modes were shown, as well as the left-turn demand for each hour. For the crash frequency graph, predicted angle crash frequencies for PPLT and permissive-only modes were plotted. 


\subsection{RESULTS}

The resulting spreadsheet from the aforementioned process provided an output table of prediction for the different phasing modes and two graphs illustrating how operations and safety varied throughout the course of a 24-hour analysis period. Examples of these can be found in the subsequent sections in Table 38, Figure 16, and Figure 17. These examples were created using input volumes from the southbound left-turn approach of the intersection of Route 220 and Route 1290 in Roanoke County, as well as the area type, number of opposing lanes, and speed limit information for this intersection. Cycle lengths, protected green ratios, subject street green ratios, and yellow plus all red times were randomized between their appropriate values to generate different timing parameter scenarios. 
Table 38 - Sample Table for Spreadsheet Tool

\begin{tabular}{|c|c|c|c|c|c|c|c|c|c|c|c|c|c|c|c|c|}
\hline Variable & & & Input & Criteria & & & & & & & & & & & & \\
\hline$\overline{\text { Area Typ }}$ & & & Urban & Urban or Rura & & & & & & & & & & & & \\
\hline Number & f Opposin & Lanes & 2 & 1,2, or 3 & & & & & & & & & & & & \\
\hline Speed Li & nit of Oppc & sing Lanes & 45 & Any value be & tween 35 & $\mathrm{mph}$ and 55 & Smph & & & & & & & & & \\
\hline & $\begin{array}{l}\text { Input } \\
\text { (vehicl }\end{array}$ & $\begin{array}{l}\text { olumes } \\
\text { s/hour) }\end{array}$ & Cycle & Protected & $\begin{array}{l}\text { Subject } \\
\text { Street }\end{array}$ & Yellow + & & & & & to-Capacity $\mathrm{R}$ & & $\begin{array}{r}\text { Average A } \\
\text { Crash F }\end{array}$ & $\begin{array}{l}\text { nnual Angle } \\
\text { requency }\end{array}$ & $\begin{array}{r}\text { Conflicts } p \\
\text { Turning }\end{array}$ & $\begin{array}{l}\text { per } 100 \text { Left- } \\
\text { Vehicles }\end{array}$ \\
\hline Hour & $\begin{array}{l}\text { Opposing } \\
\text { Volume }\end{array}$ & $\begin{array}{l}\text { Left-Turn } \\
\text { Volume }\end{array}$ & Length & Green Ratio & $\begin{array}{c}\text { Green } \\
\text { Ratio }\end{array}$ & Time & $\begin{array}{l}\text { Protected- } \\
\text { Only }\end{array}$ & $\begin{array}{l}\text { Protected- } \\
\text { Permissive }\end{array}$ & $\begin{array}{c}\text { Permissive- } \\
\text { Only }\end{array}$ & $\begin{array}{l}\text { Protected- } \\
\text { Only }\end{array}$ & $\begin{array}{l}\text { Protected- } \\
\text { Permissive }\end{array}$ & $\begin{array}{c}\text { Permissive- } \\
\text { Only }\end{array}$ & $\begin{array}{l}\text { Protected- } \\
\text { Permissive }\end{array}$ & $\begin{array}{c}\text { Permissive- } \\
\text { Only }\end{array}$ & $\begin{array}{l}\text { Protected- } \\
\text { Permissive }\end{array}$ & $\begin{array}{l}\text { Permissive- } \\
\text { Only }\end{array}$ \\
\hline 0 & 83 & 3 & 114 & 0.11 & 0.33 & 8 & 33 & & & 0.09 & & & & & & \\
\hline 1 & 61 & 0 & 222 & 0.19 & 0.76 & 7 & 263 & & & 0.00 & & & & & & \\
\hline 2 & 68 & 0 & 176 & 0.22 & 0.52 & 8 & 292 & & & 0.00 & & & & & & \\
\hline 3 & 82 & 1 & 211 & 0.21 & 0.47 & 3 & 335 & & & 0.00 & & & & & & \\
\hline 4 & 171 & 11 & 238 & 0.21 & 0.54 & 7 & 306 & & & 0.04 & & & & & & \\
\hline 5 & 468 & 13 & 112 & 0.16 & 0.47 & 2 & 227 & 422 & 365 & 0.06 & 0.03 & 0.04 & 0.065 & 0.067 & 1.05 & 2.82 \\
\hline 6 & 1093 & 39 & 217 & 0.21 & 0.33 & 3 & 345 & 370 & 57 & 0.11 & 0.11 & 0.69 & 0.064 & 0.064 & 0.00 & 0.00 \\
\hline 7 & 1642 & 104 & 125 & 0.22 & 0.46 & 3 & 317 & 376 & 58 & 0.33 & 0.28 & 1.81 & 0.064 & 0.143 & 0.00 & 8.83 \\
\hline 8 & 1415 & 108 & 212 & 0.27 & 0.35 & 3 & 441 & 460 & 34 & 0.24 & 0.23 & 3.18 & 0.064 & 0.064 & 0.00 & 0.00 \\
\hline 9 & 1021 & 119 & 198 & 0.13 & 0.54 & 6 & 153 & 344 & 237 & 0.78 & 0.35 & 0.50 & 0.093 & 0.171 & 2.81 & 10.48 \\
\hline 10 & 991 & 95 & 92 & 0.23 & 0.76 & 1 & 357 & 609 & 492 & 0.27 & 0.16 & 0.19 & 0.093 & 0.151 & 3.62 & 10.67 \\
\hline 11 & 963 & 163 & 202 & 0.15 & 0.74 & 4 & 224 & 586 & 468 & 0.73 & 0.28 & 0.35 & 0.139 & 0.248 & 5.40 & 13.14 \\
\hline 12 & 919 & 214 & 214 & 0.15 & 0.41 & 1 & 240 & 280 & 164 & 0.89 & 0.76 & 1.31 & 0.064 & 0.222 & 0.00 & 8.60 \\
\hline 13 & 984 & 212 & 130 & 0.10 & 0.46 & 1 & 138 & 199 & 184 & 1.53 & 1.07 & 1.16 & 0.130 & 0.199 & 3.66 & 7.41 \\
\hline 14 & 953 & 192 & 80 & 0.19 & 0.59 & 8 & 111 & 381 & 310 & 1.72 & 0.50 & 0.62 & 0.104 & 0.192 & 2.42 & 7.79 \\
\hline 15 & 896 & 183 & 180 & 0.12 & 0.58 & 8 & 109 & 389 & 321 & 1.68 & 0.47 & 0.57 & 0.146 & 0.222 & 5.26 & 10.10 \\
\hline 16 & 926 & 197 & 190 & 0.17 & 0.71 & 8 & 214 & 570 & 453 & 0.92 & 0.35 & 0.43 & 0.150 & 0.272 & 5.11 & 12.34 \\
\hline 17 & 947 & 209 & 204 & 0.25 & 0.75 & 7 & 370 & 676 & 494 & 0.57 & 0.31 & 0.42 & 0.127 & 0.303 & 3.50 & 13.35 \\
\hline 18 & 793 & 162 & 184 & 0.11 & 0.54 & 4 & 136 & 383 & 318 & 1.19 & 0.42 & 0.51 & 0.129 & 0.190 & 4.68 & 9.10 \\
\hline 19 & 617 & 132 & 236 & 0.08 & 0.73 & 7 & 74 & 664 & 576 & 1.77 & 0.20 & 0.23 & 0.140 & 0.197 & 6.73 & 11.74 \\
\hline 20 & 410 & 87 & 85 & 0.20 & 0.44 & 5 & 212 & 406 & 358 & 0.41 & 0.21 & 0.24 & 0.064 & 0.067 & 0.00 & 0.46 \\
\hline 21 & 324 & 31 & 239 & 0.16 & 0.58 & 7 & 226 & & & 0.14 & & & & & & \\
\hline 22 & 267 & 27 & 134 & 0.24 & 0.60 & 2 & 384 & & & 0.07 & & & & & & \\
\hline 23 & 158 & 13 & 94 & 0.10 & 0.48 & 6 & 24 & & & 0.53 & & & & & & \\
\hline
\end{tabular}




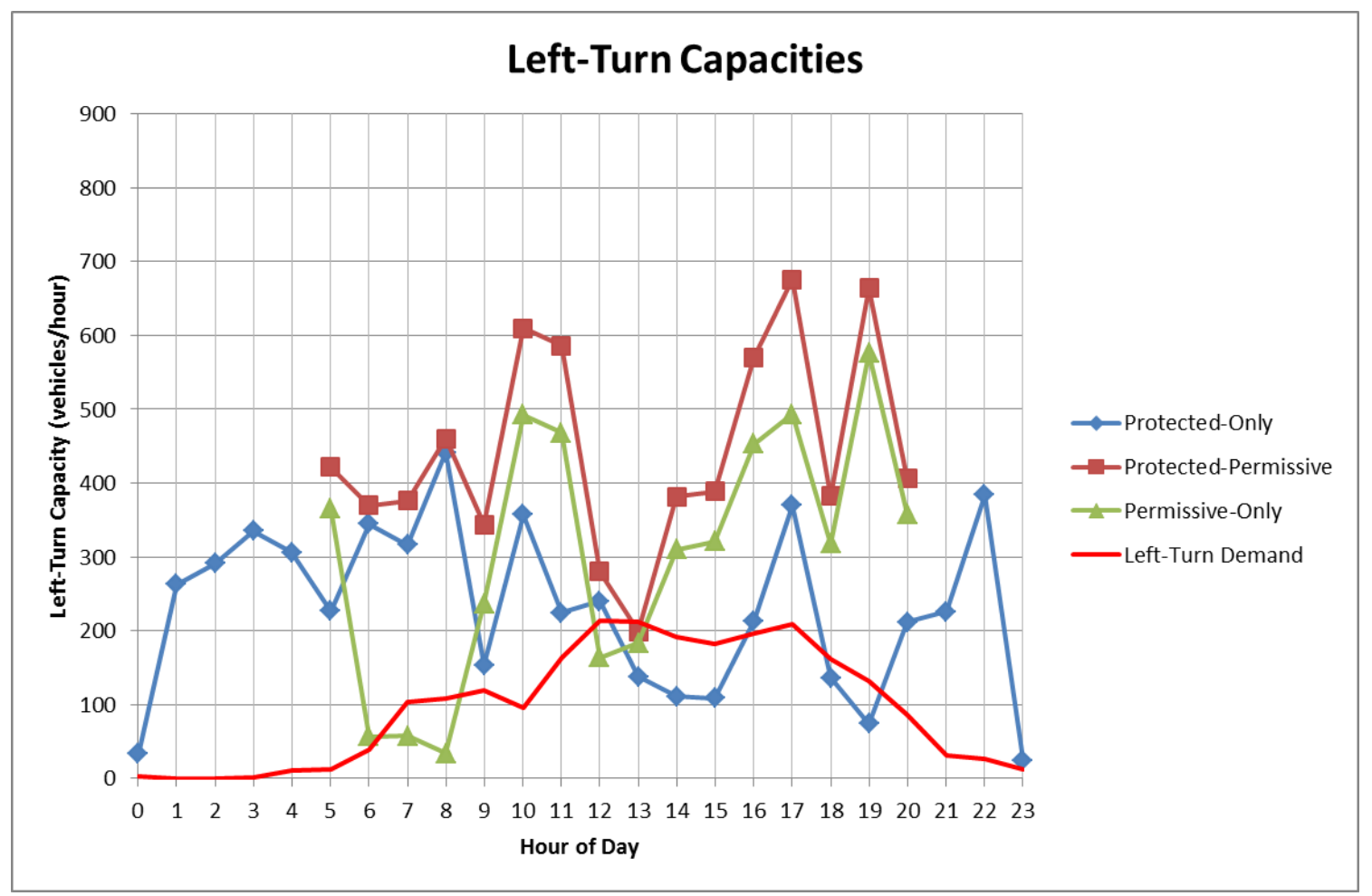

Figure 16 - Sample Capacity Plot for Spreadsheet Tool

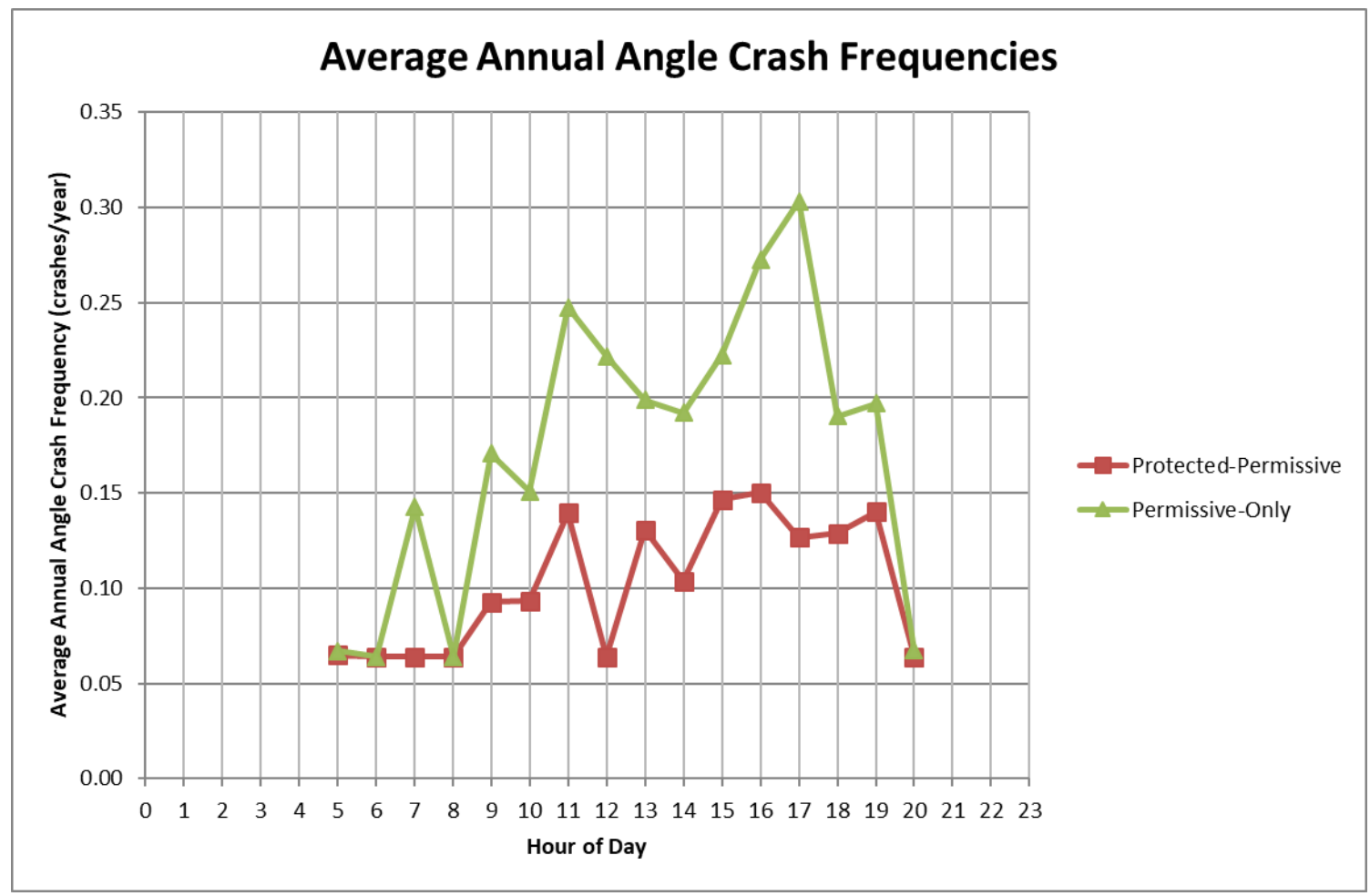

Figure 17 - Sample Crash Frequency Plot for Spreadsheet Tool 


\subsubsection{Summary of Findings}

With a tool for automatically calculating predictions for left-turn capacities, volume-tocapacity ratios, angle conflicts, and average annual angle crash frequency based on signal parameters, site characteristics, and traffic counts, engineers will now have a better way to analyze left-turn phasing control modes. Also, the spreadsheet allows engineers to visually inspect and compare predicted capacities and demands across hours of a day, and visually inspect and compare predicted angle crash frequencies across hours of a day. Currently, there are no known methods to evaluate left-turn modes for safety and operations concurrently in a time-ofday nature; therefore, this tool is the optimal technique for engineers to evaluate the time-of-day implementation of left-turn phasing.

Although the spreadsheet tool does not suggest the left-turn mode that should be used in a particular hour of the day, practitioners will be able to make a more informed decision using their own judgement regarding locational conditions and motives for the evaluation of the turn phasing (i.e. more capacity is desired, therefore turn phasing that provide additional capacity is being explored). Additionally, as this tool is intended for the use of VDOT traffic engineers, phasing mode choice should not be, and is not, prescriptive from the spreadsheet tool, since it is the intent of the VDOT Traffic Engineering Division to provide information to engineers to allow flexibility in left-turn mode determination. 


\section{CHAPTER 7: CONCLUSIONS AND RECOMMENDATIONS}

\subsection{CONCLUSIONS AND DISCUSSION}

\subsubsection{Safety Effects of Signal Conversions from PPLT to PPLT-FYA}

Converting left-turn signal displays from PPLT to PPLT-FYA can produce significant safety benefits for the intersections. From the results of this study, up to a $38 \%$ reduction in the target crash type of angle crashes can be realized for all severity types. Additionally, angle crashes for fatal and injury crash severities can be reduced by up to $37 \%$, due to the severe nature of angle crashes. These results support the findings of past studies that the FYA display is better understood than the traditional green ball for the permissive portion of PPLT phasing.

\subsubsection{Safety Effect of Signal Conversions from Protected-Only to PPLT-FYA}

Even with the implementation of FYA, protected-only to PPLT conversions can degrade safety at intersections. This could be expected, however, as the PPLT mode is less restrictive than protected-only, leading to more opportunity for human error. To quantify the safety impacts of this conversion type, this study determined that angle crashes will increase by $156 \%$ at intersections that undergo the conversion, in terms of all crash severities. Fatal and injury severities were found to increase by a larger amount, with a $230 \%$ increase in these crash severities for angle crash types. The results from this study support the notion that converting from a highly-restrictive to a less-restrictive left-turn mode will result in a significant increase in crashes, especially angle crashes, as these are typically left-turning crashes.

\subsubsection{Simulation Models}

Simulation results were used to successfully create models to predict capacity and 
conflicts for permissive-only and PPLT left-turn control modes. The models were found to have several predictor variables, such as different signal timing, traffic volume, and intersection characteristic parameters. Good model fit was found for the capacity models, although the conflict models' fit was not as good. Both the capacity and conflict models were successful in providing a method to evaluate time-of-day safety and operations. In addition to these models, a crash prediction model was found to provide acceptable crash risk by time-of-day. This model proved to match the data for Virginia intersections significantly better than previously developed models.

\subsubsection{Decision Support Tool}

A spreadsheet tool to assist engineers in the time-of-day evaluation of left-turn modes was successfully developed using models determined in this study. The tool requests signal timing, volume, and intersection parameters, and outputs the predicted capacities and safety risk for each hour of the day, both as a table and as a set of graphs. As there have been no prior tools to help with this decision in Virginia, the support tool will allow for optimal left-turn control mode choice based on changing conditions throughout the course of a day.

\subsection{RECOMMENDATIONS}

Based on the results of this study, the following recommendations can be made:

1. Crash modification factors for protected-only to protected-permissive with flashing yellow arrow should be used as an initial safety analysis method. The CMFs developed by this study are useful in determining the overall effect that a signal conversion from protected-only to PPLT with FYA phasing will make. Therefore, 
evaluating these factors should be the first step an engineer takes in determining if the increase in crashes on a yearly basis for a typical conversion is acceptable. Given that crashes are expected to increase, this information can be used in conjunction with operational analysis to determine if net benefits merit the conversion.

2. Flashing yellow arrow left-turn indications should continue to replace traditional green ball displays for protected-permissive left-turn phasing. As indicated by several past studies and the results from the crash modification factors developed by this study, flashing yellow arrows have improve safety over the green ball indication. CMFs developed in this document expanded upon currently published CMFs for the conversion by evaluating angle crash types and separate intersection configurations. Additionally, past studies have shown that the reduction in crashes can outweigh the conversion costs in most cases. Because of this, VDOT should continue to replace green ball displays for PPLT left-turn modes with FYA to improve the safety of signalized left-turns.

3. The spreadsheet tool developed by this study should be used to help guide engineers in the time-of-day analysis of left-turn control modes. Implemented using the prediction models developed in this report, the spreadsheet tool consolidates capacity and crash prediction into one worksheet, allowing for the two to be evaluated concurrently. Additionally, the tool enables time-of-day analysis of left-turn modes to be completed, so that signalized intersections can be designed to have the optimal left-turn control mode throughout the course of the day, based on changing conditions. 
4. Conflict models for protected-permissive and permissive-only left-turn modes should be used in isolation when the relative safety of the two needs to be compared, or if the effects of different parameters on the safety of the mode are to be evaluated. Since the number of left-turn conflicts is not a commonly-used safety measure, the conflict models should be used without other predictions when comparing the relative safety of permissive-only and PPLT modes. This allows for the quantification of the change in safety of a conversion from one mode to the other. Additionally, the models can be used to evaluate the relative safety effect that a parameter has, using the coefficients from the models.

5. The risk assessment model for protected-permissive and permissive-only left-turn modes should be used in conjunction with the conflict prediction models. With the lack of an existing method to determine safety effects based on surrogate measures, the risk assessment model developed addresses the gap. This method could be used in the evaluation of a newly-installed traffic signal at a location to ensure the signal will not have major safety concerns. With the signal timing, traffic volume, and site characteristic information, engineers can use the conflict prediction models and the risk assessment model to evaluate the safety of an intersection. A limitation to this, however, is that the model is currently a relative risk measure, therefore accurate crash frequencies cannot be determined.

\subsection{LIMITATIONS AND FUTURE RESEARCH}

Further research that can be done from this study includes the further development of additional CMFs, evaluation of additional simulation parameters, determination of delay 
predictions based on simulated data, examining the impact of operations on safety of phasing modes, evaluating intersection-level effects left-turn phasing modes, evaluation of field data collection methods to predict crashes based on conflicts, and advancing the spreadsheet tool for practitioners.

The largest limitation of the computation of CMFs was the number of sites found in Virginia that underwent a conversion from either green ball or protected-only to PPLT with FYA. Possible remedies for this lack of data would be to include sites from other states, as well as to re-evaluate the data source which identified locations that underwent the conversion. With the re-evaluation of sites, more sites could be added to the data set that were previously overlooked, and with including other states' data, a broader geographic area could be represented.

In considering changes to the data available in Virginia only, waiting for more available data could increase the number of sites analyzed because more sites could have been converted and more AADT data would be available. When identifying the locations to be analyzed in this report, 63 sites were not considered since they were only planned and not built, and 43 additional intersections were not considered since they did not have complete AADT data. If in the future more of the planned sites are converted to FYA from green ball or protected-only and more traffic count data is available, a potential for up to 106 more sites could be analyzed, depending on whether they are conversions from green ball or protected-only to FYA PPLT specifically, resulting in 142 total analysis sites. With more sites, the analysis should produce narrower confidence intervals and more statistically significant results. Additionally, with more data in the after period of signal conversions, sites could be evaluated to determine if there is a learning curve over time when converting from protected-only to PPLT. This would be useful to understand if drivers comprehend the proper actions for permissive left-turns at newly-converted 
sites.

For the prediction models developed by the simulation data, capacities and conflicts can be calculated with a high level of confidence, but there is still some uncertainty in the results. Further evaluation of simulation scenarios could assist in explaining some of the variability in the models. Additional parameters should be modeled in future iterations of the simulation models. These parameters could include friction factors such as pedestrians and bicyclists, the effects of vehicle arrivals in platoons simulated by signal coordination, and different intersection geometries. If additional simulation runs are created with new parameters, the effects of more parameters in the determination of capacities and conflicts could be evaluated.

Another prediction model that could be developed in the future would be for left-turn vehicle delay. If this is done, safety and operations could be compared by using dollar values assigned to delay and crashes. This could not be done before, as the monetary effects from the increase in capacity are ambiguous. Assigning dollar values to the value of travel time and cost of an angle crash could be combined with the predictions to evaluate the impacts of PPLT and permissive-only relative to protected-only phasing, on a cost-to-benefit basis. This could help in the determination of phasing mode, as a quantitative evaluation metric could be created.

With the knowledge of how vehicle delay and capacities are affected by left-turn modes and other parameters, safety could be evaluated as a function of operations. It is typically assumed that the longer a vehicle waits to make a permitted left-turn, the more willing they are to accept smaller gaps in opposing traffic. This should be quantified in future research to have a better understanding of the impact of permissive capacity on safety under high demand volumes.

Other limitations of the operational and safety predictions for left-turn mode are the lack of knowledge of how other approaches are impacted, and not understanding how vehicle 
composition affects the measures. A large part in the determination of phasing is how the intersection performs as a whole; therefore, intersection-level capacity and delay effects should be evaluated in later iterations of this research. Additionally, the study assumed zero heavy vehicles in the simulations, therefore, the effects of these types of vehicles was not studied. In later studies, the percentages of heavy vehicles should be varied to better understand the parameter's impact.

In furthering the usefulness of the crash prediction model based on conflicts, the evaluation of field collection techniques on the prediction of crashes could be evaluated. Fieldgathered conflict data could provide a short-term safety assessment method to engineers when historical crash data is unavailable. This would be useful in the new installation of traffic signals, and would take into consideration the effects of real-world driver behavior. Also, the crash prediction models could be redeveloped using more refined datasets, such as separating by intersection configuration. In doing this, more precise models could be developed, as it was found that the model developed in this study varied in performance across intersection types.

Finally, the spreadsheet tool developed in this study could be further improved upon by adding delay calculations and evaluating the crash costs and delay benefits of different left-turn phasing modes, as well as including estimates of uncertainty on conflict and capacity predictions. By improving upon the tool, engineers would have more metrics to evaluate the modes so that they can make a better-informed phasing mode determination. Additionally, the cost-benefit analysis of the modes could help to provide recommended phasing modes to the engineer, although other factors may lead them to choose a different left-turn mode. With the implementation of benefit and cost metrics, the evaluation of the time-of-day conversion of a signal could be completed in instances where time-of-day PPLT is only needed for a couple of 
hours per day. This would aid the engineer in deciding if the benefits of converting the signal for only a couple of hours of use outweigh the overall costs of the change. In including uncertainty estimates for the predictions made by the models in the spreadsheet tool, the engineers could also better understand the accuracy of the results and realize that capacity predictions for different left-turn modes may not be significantly different from one another. 


\section{REFERENCES}

1. Schattler, K. L., E. Anderson, and T. Hanson. Safety Evaluation of Flashing Yellow Arrows for Protected/Permissive Left-Turn Control. Illinois Department of Transportation, Springfield, Volume 3 FInal Report 2016.

2. Simpson, C. L., and S. A. Troy. Safety Effectiveness of Flashing Yellow Arrow: Evaluation of 222 Signalized Intersections in North Carolina. Transportation Research Record: Journal of the Transportation Research Board, Vol. 2492, no. 05, 2015, pp. 46-56. DOI: $10.3141 / 2492-05$

3. Federal Highway Administration. Manual on Uniform Traffic Control Devices: 2009 Edition. Federal Highway Administration, Washington, D.C., United States of America, 2009.

4. VDOT Traffic Engineering Division. Guidance for Determination and Documentation of Left-Turn Phasing Mode. Virginia Department of Transportation, Version 1.0 2015.

5. Noyce, D. A., and K. C. Kacir. Driver's Understanding of Protected/Permitted Left-Turn Signal Displays. Transportation Research Record: Journal of the Transportation Research Board, January 2001.

6. Brehmer, C. L., K. C. Kacir, D. A. Noyce, and M. P. Manser. Evaluation of Traffic Signal Displays for Protected/Permissive Left-Turn Control. Transportation Research Board, Washington, D.C., NCHRP Report 4932003.

7. Knodler, M. A. Evaluation of Traffic Signal Displays for Protected-Permissive Left-Turn Control Using Driving Simulator Technology. Journal of Transportation Engineering, Vol. 131, no. 4, April 2005, pp. 270-278.

8. Reitgraf, A., and K. L. Schattler. Behavior of Left-Turning Drivers During Permissive 
Interval of Protected-Permissive Operation: Effect of Signal Display. Transportation Research Record: Journal of the Transportation Research Board, Vol. 2384, no. 5, 2013, pp. 35-44. DOI: $10.3141 / 2384-05$

9. Rescot, R. A., S. Qu, R. Noteboom, and A. Nafakh. Evaluation of Flashing Yellow Arrow Traffic Signals in Indiana. Indiana Department of Transportation, Indianapolis, Final Report 2015.

10. Pulugurtha, S. S., M. Agurla, and K. S.C. Khader. How Effective are "Flashing Yellow Arrow" Signals in Enhancing Safety? in Transportation and Development Institute, Chicago, 2011, pp. 1096-1104.

11. QI, Y., M. Zhang, Y. Wang, and X. Chen. Safety Performance of Flashing Yellow Arrow Signal Indication. Transportation Research Record: Journal of the Transportation Research Board, 2012.

12. Lin, P.-S., A. Fabregas, and E. Gonzalez-Velez. Assessment of a Flashing Yellow Arrow Signal Implementation using Gap Acceptance Measures. Sustainable Transportation Systems, 2012, pp. 341-348.

13. Srinivasan, R., C. Lyon, B. Persaud, J. Baek, F. Gross, S. Smith, and C. Sundstrom. Crash Modification Factors for Changes to Left-Turn Phasing. Transportation Research Record: Journal of the Transportation Research Board, Vol. 2279, 2012, pp. 108-117. DOI: $10.3141 / 2279-13$

14. Navarro, A. K. An Analysis of the Protected-Permitted Left-Turn at Intersections with a Varying Number of Opposing Through Lanes. University of Central Florida, Orlando, Master's Thesis 2014. 
15. Qi, Y., X. Chen, A. Guo, and L. Yu. Protected-Permissive Left-Turn Signal Control Mode: New Analytical Approach to Estimate Operational Benefit and Safety Cost. Transportation Research Record: Journal of the Transportation Research Board, Vol. 2149, no. 5, 2010, pp. 37-49. DOI: $10.3141 / 2149-05$

16. Agent, K. R. Guidelines for the Use of Protected/Permissive Left-Turn Phasing. Kentrucky Transportation Research Program, Lexington, Final Report 1985.

17. Agent, K. R., and N. Stamatiadis. Guidelines for the Installation of Left-Turn Phasing. Kentucky Transportation Center, Lexington, Final Report 1995.

18. Bonneson, J. A., and M. D. Fontaine. Engineering Study Guide for Evaluating Intersection Improvements. Transportation Research Board, Washington, D.C., NCHRP Report 4572001.

19. Zhang, L., P. Prevedouros, and H. Li. Warrants for Protected Left-Turn Phasing. Journal of Transportation Engineering, February 2005.

20. Federal Highway Administration. Traffic Signal Timing Manual. Federal Highway Administration, Washington, D.C., Final Report 2008.

21. Yu, L., Y. Qi, H. Yu, L. Guo, and X. Chen. Development of Left-Turn Operations Guidelines at Signalized Intersections. Texas Department of Transportation, Austin, Technical Report 2009.

22. Radwan, E., H. Abou-Senna, A. Navarro, and S. Chalise. Dynamic Flashing Yellow Arrow (FYA): A Study on Variable Left Turn Mode Operational and Safety Impacts. Florida Department of Transportation Research Center, Tallahassee, Final Report 2013.

23. Chalise, S., E. Radwan, and H. Abou-Senna. Analysis of Variable Left Turn Mode by Time of Day for Flashing Yellow Arrow Signals Using the Left Turn Delay Prediction Models. 
Transportation Research Record: Journal of the Transportation Research Board, 2017.

24. Davis, G. A., J. Hourdos, and V. Moshtagh. Development of Guidelines for Permitted LeftTurn Phasing Using Flashing Yellow Arrows. Minnesota Department of Transportation, St. Paul, Final Report 2015.

25. Garber, N. J., and G. Rivera. Safety Performance Functions for Intersections on Highways Maintained by the Virginia Department of Transportation. Virginia Department of Transportation, Richmond, FInal Contract 2010.

26. American Association of State Highway and Transportation Officials. Highway Safety Manual, Volume 1, 1st ed. AASHTO, Washington, D.C., United States of America, 2010.

27. Transportation Research Board. HCM 2010: Highway Capacity Manual, 2010th ed. TRB, Washington, D.C., United States of America, 2010.

28. PTV AG. PTV VISSIM 8 User Manual. PTV AG, Karlsruhe, Final Report 2015.

29. Notz, W. (2005, August) Introductory Overview Lecture on Computer Experiments - Design. Lecture. [Online]. http://citeseerx.ist.psu.edu/viewdoc/ download?doi=10.1.1.526.8650\&rep=rep1\&type=pdf. DOI: 10.1.1.526.8650

30. Gettman, D., L. Pu, T. Sayed, and S. Shelby. Surrogate Safety Assessment Model and Validation: Final Report. Federal Highway Administration, McLean, Final Report 2008. 


\section{APPENDICIES}

\section{APPENDIX A: PUBLICATIONS}

"Safety Effects of Flashing Yellow Arrows Used in Protected Permitted Phasing: Comparison of Full Bayes and Empirical Bayes Results,” Poster Presentation, Publication Pending Approval. TRB Annual Meeting, Washington, D.C., 2018. 


\section{APPENDIX B: DATA FOR CMF DEVELOPMENT}

Table B1: AADT Data

\begin{tabular}{|c|c|c|c|c|c|c|c|c|c|c|c|c|c|c|c|c|c|c|}
\hline Site & \begin{tabular}{|c|}
2016 AADT \\
Major
\end{tabular} & $\begin{array}{c}2016 \text { AADT } \\
\text { Minor }\end{array}$ & $\begin{array}{c}2015 \text { AADT } \\
\text { Major }\end{array}$ & $\begin{array}{c}2015 \text { AADT } \\
\text { Minor }\end{array}$ & $\begin{array}{c}2014 \text { AADT } \\
\text { Major }\end{array}$ & $\begin{array}{c}2014 \text { AADT } \\
\text { Minor }\end{array}$ & $\begin{array}{c}2013 \text { AADT } \\
\text { Major }\end{array}$ & $\begin{array}{c}2013 \text { AADT } \\
\text { Minor }\end{array}$ & r $\begin{array}{c}2012 \text { AADT } \\
\text { Major }\end{array}$ & $\begin{array}{c}2012 \text { AADT } \\
\text { Minor }\end{array}$ & \begin{tabular}{|c|}
2011 AADT \\
Major
\end{tabular} & $\begin{array}{c}2011 \text { AADT } \\
\text { Minor }\end{array}$ & $\begin{array}{c}2010 \text { AADT } \\
\text { Major }\end{array}$ & $\begin{array}{c}2010 \text { AADT } \\
\text { Minor }\end{array}$ & $\begin{array}{c}2009 \text { AADT } \\
\text { Major }\end{array}$ & $\begin{array}{c}2009 \text { AADT } \\
\text { Minor }\end{array}$ & $\begin{array}{c}2008 \text { AADT } \\
\text { Major }\end{array}$ & $\begin{array}{c}2008 \text { AADT } \\
\text { Minor }\end{array}$ \\
\hline 7 & 22823 & 6815.5 & 22099.5 & 7876 & 20951.5 & 7565.5 & 20445.5 & 7460 & 19091.5 & 7256.5 & 19294.5 & 7290 & 19631.5 & 7429 & 19179.5 & 6877 & 17757 & 7293 \\
\hline 10 & 12673 & 2912 & 15137 & 2933 & 14351 & 2817 & 14004 & 2778 & 13440 & 2739 & 13583 & 2751 & 13820 & 2804 & 12634 & 2743 & 11697 & 2909 \\
\hline 14 & 9740 & 4444 & 8941 & 3893 & 8589 & 3739 & 8469 & 3687 & 10141 & 4255 & 10187 & 4274 & 10382 & 4356 & 10239 & 4393 & 10859 & 4658 \\
\hline 15 & 7425 & 3404 & 8551 & 3294 & 8214 & 3164 & 8099 & 3120 & 7774 & 3189 & 7810 & 3204 & 7959 & 3265 & 7278 & 3646 & 7718 & 3867 \\
\hline 21 & 8855 & 2533.5 & 8551.5 & 2447 & 8786 & 2429.5 & 8681.5 & 2401 & 8699.5 & 2406 & 8890.5 & 2089 & 9004.5 & 2115.5 & 8943 & 2101 & 8049.5 & 2128.5 \\
\hline 36 & 9287 & 5511 & 9919 & 5591 & 9628.5 & 5427 & 9513 & 5362 & 9490 & 5622 & 9591 & 5682 & 9758.5 & 5781 & 9660 & 4882 & 10034.5 & 5072 \\
\hline 42 & 4409 & 3841 & 3940 & 3744 & 3825 & 3635 & 3779 & 3591 & 3768 & 3481 & 3808 & 3519 & 3875 & 3580 & 3547 & 3487 & 3684 & 3622 \\
\hline 43 & 18463.5 & 3255 & 17726 & 3076.5 & 17499.5 & 2983.5 & 17662 & 2942 & 18260.5 & 2712.5 & 18074.5 & 2740.5 & 18220.5 & 2772.5 & 17629.5 & 2642 & 17448 & 2681.5 \\
\hline 48 & 5552 & 2420 & 6121 & 2419 & 5942 & 2348 & 5870.5 & 2320 & 5704 & 2177 & 5764.5 & 2201 & 5865.5 & 2239 & 5542 & 2331 & 5756.5 & 2422 \\
\hline 52 & 7804 & 553 & 7524 & 534 & 8131 & 517 & 8146 & 528.5 & 8150 & 530 & 8222.5 & 535 & 8067.5 & 549 & 8248.5 & 551 & 8123 & 541.5 \\
\hline 55 & 4519 & 4088 & 4364 & 3948 & 4363 & 4045.5 & 4311.5 & 3997.5 & 4320.5 & 4005.5 & 4443.5 & 3653 & 4500.5 & 3700 & 4470 & 3675 & 4396.5 & 4252 \\
\hline 62 & 24544 & 14503 & 23254 & 13664 & 22817 & 13408 & 22790 & 13392 & 20747 & 13192 & 20861 & 13265 & 21039 & 13378 & 17857 & 9839 & 18680 & 10641 \\
\hline 64 & 15749 & 4966 & 14497 & 4666 & 14225 & 4578 & 14208 & 4573 & 14020.5 & 4557 & 14097.5 & 4582 & 14218 & 4621 & 10170.5 & 4698 & 10848 & 5081 \\
\hline 70 & 7935 & 3780 & 8127 & 3329 & 7841 & 3212 & 7719 & 3162 & 7198 & 3167 & 7126 & 3136 & 7191 & 3164.5 & 6903 & 3394 & 7224 & 3551.5 \\
\hline 78 & 26005 & 3258 & 24322 & 3201 & 24322 & 3141 & 24322 & 3137 & 26248 & 3300 & 26344 & 3318 & 26528 & 3346 & 24961 & 3334 & 25151 & 3488 \\
\hline 79 & 21872.5 & 5481 & 21167.5 & 5916 & 20632 & 5805 & 20456.5 & 5798 & 20318.5 & 5205 & 20116 & 5233 & 20299.5 & 5278 & 20899.5 & 5602 & 19775.5 & 5860 \\
\hline 91 & 5435 & 3161 & 5383 & 3131 & 5499 & 3350 & 5494 & 3316.5 & 5554 & 3352.5 & 5760 & 3564 & 5333 & 3300 & 5815 & 3598.5 & 7102 & 4202 \\
\hline 93 & 12504 & 3545 & 12385 & 3511.5 & 13900 & 3791 & 13897 & 3789.5 & 14040 & 3829 & 12676 & 3884 & 13150 & 3907 & 12745 & 3909.5 & 13646 & 4313 \\
\hline 94 & 7035 & 6844 & 6968 & 6778.5 & 7709 & 7075 & 7702 & 7069 & 7786 & 7146 & 8201 & 6902.5 & 7593 & 6390.5 & 8280 & 6968.5 & 8625.5 & 8506 \\
\hline 99 & 14992.5 & 5971 & 14797 & 5914 & 13976 & 6456 & 13649 & 6450 & 13429.5 & 6521 & 14375.5 & 9431 & 14545 & 8732 & 14587.5 & 9522 & 13805 & 7082 \\
\hline 116 & 27795 & 8820.5 & 27618 & 8781 & 27622 & 8831 & 26599 & 8828.5 & 26489 & 8919.5 & 26949 & 7859 & 27953 & 8062 & 29115 & 8363.5 & 28976 & 9268.5 \\
\hline 138 & 13105 & 11475 & 12823.5 & 11228 & 13678.5 & 11583 & 13520.5 & 11449 & 13946.5 & 11810 & 14344 & 10798 & 14466.5 & 10890 & 14436 & 10867 & 14730.5 & 10837 \\
\hline 139 & 8469 & 4240.5 & 8335.5 & 4436.5 & 8137 & 4331 & 8066 & 4293 & 8463.5 & 4194 & 8591 & 4257 & 8808.5 & 4365 & 8240 & 4700 & 8621 & 4917.5 \\
\hline 141 & 8431 & 1772 & 8962 & 2077 & 9131 & 2020 & 9284 & 2001 & 9743 & 1981 & 9583 & 1962 & 9398 & 1943 & 10475 & 1923 & 10315 & 1904 \\
\hline 142 & 7624.5 & 3867.5 & 7783 & 3648 & 7929.5 & 3540 & 8062.5 & 3511 & 8670 & 3389.5 & 8527.5 & 3268 & 8363 & 3147 & 9261 & 3025.5 & 9119 & 2904 \\
\hline 144 & 8093 & 1532 & 8781 & 2151 & 8948 & 2769 & 9096 & 2704 & 9310 & 2639 & 9157 & 2574 & 8980 & 2508 & 9200 & 2443 & 9059 & 2378 \\
\hline 147 & 5236 & 2267 & 5123 & 2218 & 6660 & 2086 & 6648 & 2094 & 6782 & 2127 & 6958 & 2937 & 7120 & 3011 & 6936 & 2768 & 7196 & 2872 \\
\hline 176 & 11039 & 10012 & 10949.5 & 9931 & 10648 & 10461 & 10760 & 10571 & 10929 & 10736.5 & 9722 & 8678 & 9962 & 8892 & 9821.5 & 8767 & 10349.5 & 7882 \\
\hline 179 & 16287 & 7849.5 & 15743.5 & 7587.5 & 15572.5 & 7505 & 16189.5 & 7599.5 & 16378 & 7051 & 16471 & 6501.5 & 14650.5 & 6116.5 & 13935.5 & 5818 & 14024.5 & 5855 \\
\hline 259 & 7736 & 7040 & 6994 & 6611.5 & 7173.5 & 5487.5 & 7108 & 4363 & 7514 & 4340.5 & 7446 & 4317.5 & 7378 & 4295 & 6737 & 4278 & 6615 & 4260.5 \\
\hline 265 & 34873 & 1962 & 34740 & 1958 & 34606 & 1953 & 34473 & 1949 & 38515 & 1945 & 38367 & 1940 & 38219 & 1936 & 39012 & 1928 & 37093 & 1919 \\
\hline 266 & 16387 & 4890 & 15402 & 4943 & 15096 & 4863 & 15654 & 5002.5 & 16373 & 4987 & 16743 & 5073 & 16723 & 5064 & 17189 & 4965 & 17890 & 5125.5 \\
\hline 273 & 10763 & 5464.5 & 10349 & 5390 & 9958 & 5335 & 10124 & 5411 & 9985 & 5381.5 & 9935 & 5371 & 8972 & 5303.5 & 8822 & 5269.5 & 9667 & 4988 \\
\hline 275 & 9205.5 & 2277.5 & 8869 & 2200 & 8577.5 & 2143 & 8715 & 2148 & 8595.5 & 2118.5 & 8552.5 & 2108 & 7392 & 2089.5 & 7255 & 2045 & 7644 & 2021 \\
\hline 277 & 29807 & 1923 & 27644 & 2244 & 27087 & 2180 & 26489 & 2141 & 25118 & 2776 & 25305 & 2762 & 25396 & 2763 & 26266 & 2770 & 25964 & 2942 \\
\hline 279 & 8765.5 & 4159.5 & 8521.5 & 4018.5 & 8318 & 3914.5 & 8839 & 3855.5 & 8867.5 & 3803 & 8790.5 & 3784 & 9033.5 & 3933 & 8610.5 & 3848.5 & 8499 & 3803.5 \\
\hline 283 & 33598 & 7582.5 & 32424 & 7317.5 & 31792.5 & 7175 & 32773.5 & 6972 & 32589.5 & 6933 & 33327.5 & 7090 & 31174 & 7516.5 & 29938 & 7219 & 29188.5 & 7038 \\
\hline
\end{tabular}


Table B2: Intersection Characteristics for Base CMFs

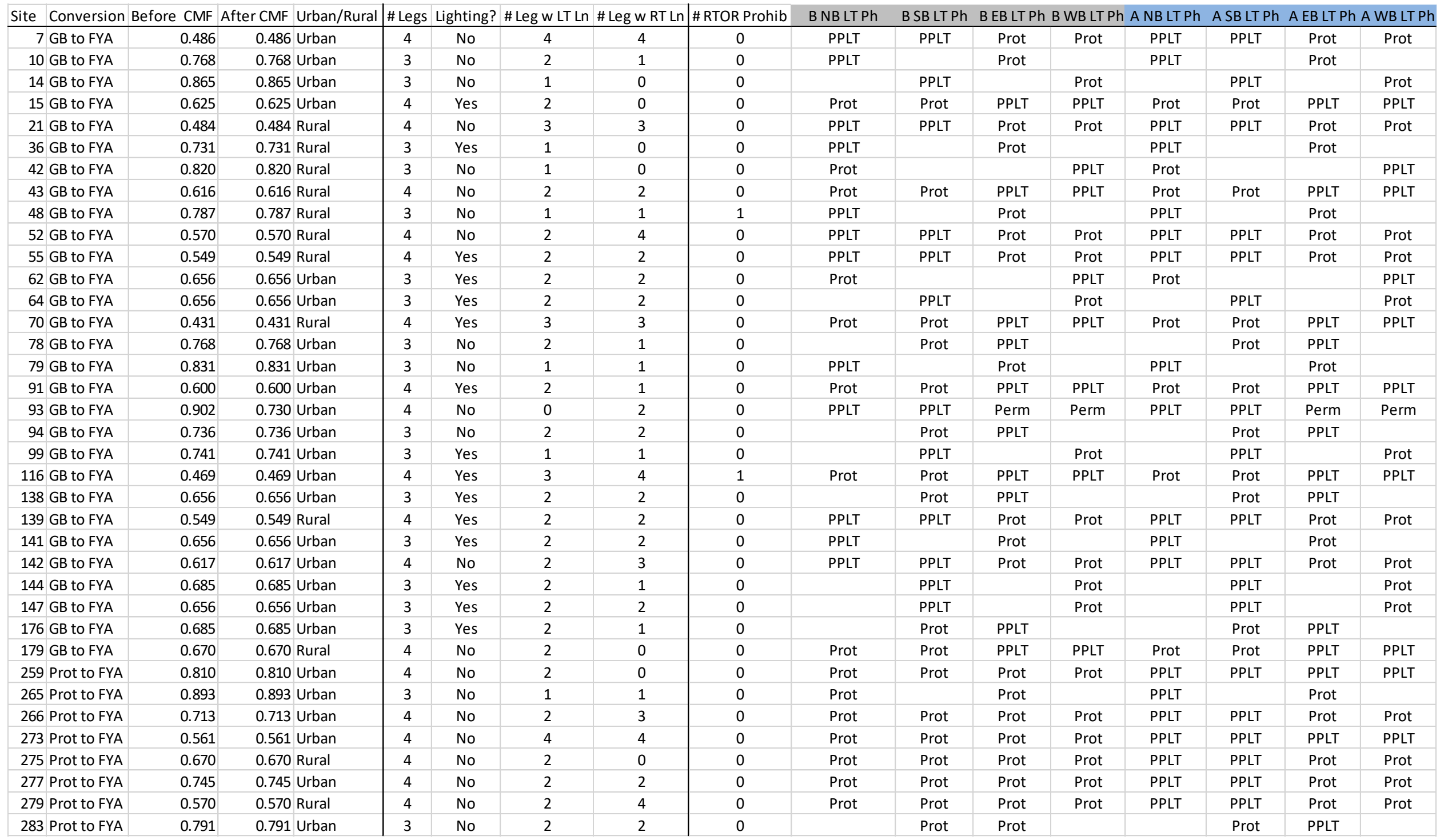


Table B3: Crashes for Before Period Years

\begin{tabular}{|c|c|c|c|c|c|c|c|c|c|c|c|c|c|c|c|c|c|c|}
\hline \multirow[b]{2}{*}{ Site } & \multicolumn{6}{|c|}{ Year } & \multicolumn{6}{|c|}{ Total Crashes } & \multicolumn{6}{|c|}{ FI Crashes } \\
\hline & B5 & B4 & B3 & B2 & B1 & $\mathrm{P} \_\mathrm{Bx}$ & B5 & B4 & B3 & B2 & B1 & P_Bx & B5 & B4 & B3 & B2 & B1 & P_Bx \\
\hline 7 & 2010 & 2011 & 2012 & 2013 & 2014 & & 9 & 13 & 6 & 11 & 9 & & 1 & 6 & 1 & 3 & 2 & \\
\hline 10 & 2010 & 2011 & 2012 & 2013 & 2014 & & 0 & 0 & 2 & 0 & 0 & & 0 & 0 & 2 & 0 & 0 & \\
\hline 14 & 2010 & 2011 & 2012 & 2013 & 2014 & & 0 & 2 & 0 & 3 & 1 & & 0 & 1 & 0 & 2 & 0 & \\
\hline 15 & 2010 & 2011 & 2012 & 2013 & 2014 & & 1 & 4 & 2 & 1 & 2 & & 0 & 1 & 0 & 1 & 1 & \\
\hline 21 & 2010 & 2011 & 2012 & 2013 & 2014 & & 5 & 1 & 6 & 3 & 3 & & 2 & 0 & 1 & 2 & 1 & \\
\hline 36 & 2009 & 2010 & 2011 & 2012 & 2013 & 2014 & 2 & 2 & 1 & 3 & 0 & 0 & 0 & 1 & 1 & 2 & 0 & 0 \\
\hline 42 & 2009 & 2010 & 2011 & 2012 & 2013 & 2014 & 3 & 0 & 0 & 2 & 0 & 4 & 2 & 0 & 0 & 1 & 0 & 1 \\
\hline 48 & 2010 & 2011 & 2012 & 2013 & 2014 & 2015 & 1 & 0 & 2 & 1 & 1 & 1 & 0 & 0 & 1 & 0 & 0 & 0 \\
\hline 52 & 2010 & 2011 & 2012 & 2013 & 2014 & & 1 & 3 & 0 & 1 & 2 & & 0 & 1 & 0 & 1 & 0 & \\
\hline 55 & 2010 & 2011 & 2012 & 2013 & 2014 & & 2 & 0 & 0 & 2 & 0 & & 0 & 0 & 0 & 1 & 0 & \\
\hline 62 & 2009 & 2010 & 2011 & 2012 & 2013 & 2014 & 6 & 4 & 3 & 6 & 8 & 1 & 4 & 1 & 3 & 3 & 5 & 1 \\
\hline 64 & 2009 & 2010 & 2011 & 2012 & 2013 & 2014 & 3 & 2 & 0 & 1 & 2 & 0 & 0 & 0 & 0 & 1 & 0 & 0 \\
\hline 70 & 2009 & 2010 & 2011 & 2012 & 2013 & 2014 & 1 & 0 & 1 & 0 & 1 & 1 & 0 & 0 & 0 & 0 & 0 & 0 \\
\hline 78 & 2008 & 2009 & 2010 & 2011 & 2012 & 2013 & 4 & 4 & 1 & 7 & 4 & 1 & 1 & 2 & 0 & 3 & 2 & 1 \\
\hline 79 & 2011 & 2012 & 2013 & 2014 & 2015 & 2016 & 3 & 4 & 5 & 3 & 3 & 0 & 1 & 3 & 0 & 2 & 2 & 0 \\
\hline 93 & 2010 & 2011 & 2012 & 2013 & 2014 & & 9 & 1 & 5 & 2 & 2 & & 2 & 1 & 2 & 2 & 0 & \\
\hline 94 & 2010 & 2011 & 2012 & 2013 & 2014 & & 0 & 3 & 2 & 3 & 1 & & 0 & 0 & 2 & 1 & 1 & \\
\hline 99 & 2010 & 2011 & 2012 & 2013 & 2014 & & 8 & 7 & 7 & 4 & 6 & & 4 & 2 & 4 & 2 & 3 & \\
\hline 116 & 2008 & 2009 & 2010 & 2011 & 2012 & 2013 & 8 & 19 & 18 & 22 & 22 & 9 & 3 & 6 & 7 & 2 & 7 & 3 \\
\hline 138 & 2009 & 2010 & 2011 & 2012 & 2013 & 2014 & 3 & 3 & 2 & 1 & 4 & 1 & 0 & 0 & 0 & 0 & 2 & 0 \\
\hline 139 & 2010 & 2011 & 2012 & 2013 & 2014 & 2015 & 3 & 3 & 4 & 0 & 1 & 0 & 1 & 2 & 0 & 0 & 1 & 0 \\
\hline 141 & 2010 & 2011 & 2012 & 2013 & 2014 & 2015 & 2 & 2 & 2 & 0 & 0 & 0 & 0 & 1 & 2 & 0 & 0 & 0 \\
\hline 142 & 2009 & 2010 & 2011 & 2012 & 2013 & 2014 & 4 & 8 & 1 & 0 & 3 & 1 & 3 & 5 & 0 & 0 & 1 & 0 \\
\hline 144 & 2010 & 2011 & 2012 & 2013 & 2014 & 2015 & 3 & 3 & 0 & 0 & 1 & 0 & 0 & 2 & 0 & 0 & 0 & 0 \\
\hline 147 & 2010 & 2011 & 2012 & 2013 & 2014 & 2015 & 1 & 0 & 2 & 0 & 1 & 0 & 1 & 0 & 0 & 0 & 0 & 0 \\
\hline 176 & 2008 & 2009 & 2010 & 2011 & 2012 & 2013 & 4 & 3 & 6 & 5 & 7 & 4 & 2 & 2 & 2 & 1 & 3 & 3 \\
\hline 179 & & 2008 & 2009 & 2010 & 2011 & 2012 & & 8 & 5 & 9 & 8 & 5 & & 0 & 4 & 1 & 2 & 2 \\
\hline 259 & 2009 & 2010 & 2011 & 2012 & 2013 & 2014 & 1 & 3 & 3 & 0 & 2 & 1 & 1 & 0 & 2 & 0 & 1 & 0 \\
\hline 265 & 2009 & 2010 & 2011 & 2012 & 2013 & 2014 & 2 & 1 & 2 & 2 & 3 & 0 & 1 & 0 & 0 & 2 & 0 & 0 \\
\hline 266 & 2010 & 2011 & 2012 & 2013 & 2014 & & 5 & 5 & 10 & 15 & 18 & & 4 & 4 & 5 & 4 & 9 & \\
\hline 273 & 2010 & 2011 & 2012 & 2013 & 2014 & 2015 & 5 & 0 & 0 & 10 & 3 & 3 & 3 & 0 & 0 & 4 & 3 & 2 \\
\hline 279 & 2010 & 2011 & 2012 & 2013 & 2014 & 2015 & 3 & 1 & 2 & 0 & 3 & 7 & 1 & 1 & 0 & 0 & 1 & 1 \\
\hline 283 & 2009 & 2010 & 2011 & 2012 & 2013 & 2014 & 5 & 2 & 2 & 1 & 2 & 3 & 1 & 0 & 0 & 0 & 0 & 2 \\
\hline
\end{tabular}


Table B4: Crashes for After Period Years

\begin{tabular}{|c|c|c|c|c|c|c|c|c|c|c|c|c|c|c|c|c|c|c|}
\hline \multirow[b]{2}{*}{ Site } & \multicolumn{6}{|c|}{ Year } & \multicolumn{6}{|c|}{ Total Crashes } & \multicolumn{6}{|c|}{ FI Crashes } \\
\hline & P_Ax & A1 & $\mathrm{A} 2$ & A3 & A4 & A5 & P_Ax & $\mathrm{A} 1$ & A2 & A3 & A4 & A5 & P_Ax & $\mathrm{A} 1$ & A2 & A3 & A4 & A5 \\
\hline 7 & 2015 & 2016 & & & & & 7 & 9 & & & & & 3 & 4 & & & & \\
\hline 10 & 2015 & 2016 & & & & & 1 & 1 & & & & & 1 & 1 & & & & \\
\hline 14 & 2015 & 2016 & & & & & 0 & 2 & & & & & 0 & 1 & & & & \\
\hline 15 & 2015 & 2016 & & & & & 3 & 1 & & & & & 1 & 1 & & & & \\
\hline 21 & 2015 & 2016 & & & & & 1 & 1 & & & & & 0 & 0 & & & & \\
\hline 36 & & 2015 & 2016 & & & & & 1 & 2 & & & & & 0 & 2 & & & \\
\hline 42 & & 2015 & 2016 & & & & & 3 & 0 & & & & & 1 & 0 & & & \\
\hline 43 & & 2015 & 2016 & & & & & 5 & 2 & & & & & 3 & 2 & & & \\
\hline 48 & 2015 & 2016 & & & & & 0 & 0 & & & & & 0 & 0 & & & & \\
\hline 52 & 2015 & 2016 & & & & & 1 & 2 & & & & & 0 & 1 & & & & \\
\hline 55 & 2015 & 2016 & & & & & 0 & 1 & & & & & 0 & 1 & & & & \\
\hline 62 & 2014 & 2015 & 2016 & & & & 3 & 5 & 4 & & & & 2 & 2 & 2 & & & \\
\hline 64 & 2014 & 2015 & 2016 & & & & 0 & 1 & 0 & & & & 0 & 0 & 0 & & & \\
\hline 70 & 2014 & 2015 & 2016 & & & & 0 & 1 & 2 & & & & 0 & 0 & 0 & & & \\
\hline 78 & 2013 & 2014 & 2015 & 2016 & & & 1 & 6 & 8 & 4 & & & 0 & 1 & 1 & 1 & & \\
\hline 79 & 2016 & & & & & & 3 & & & & & & 1 & & & & & \\
\hline 91 & 2015 & 2016 & & & & & 3 & 1 & & & & & 1 & 0 & & & & \\
\hline 93 & 2015 & 2016 & & & & & 5 & 4 & & & & & 3 & 1 & & & & \\
\hline 94 & 2015 & 2016 & & & & & 3 & 5 & & & & & 0 & 1 & & & & \\
\hline 99 & 2015 & 2016 & & & & & 4 & 4 & & & & & 2 & 1 & & & & \\
\hline 116 & 2013 & 2014 & 2015 & 2016 & & & 3 & 6 & 21 & 12 & & & 0 & 4 & 8 & 4 & & \\
\hline 138 & 2014 & 2015 & 2016 & & & & 0 & 2 & 5 & & & & 0 & 0 & 0 & & & \\
\hline 139 & 2015 & 2016 & & & & & 2 & 3 & & & & & 0 & 1 & & & & \\
\hline 141 & 2015 & 2016 & & & & & 0 & 2 & & & & & 0 & 0 & & & & \\
\hline 142 & 2014 & 2015 & 2016 & & & & 0 & 2 & 3 & & & & 0 & 0 & 0 & & & \\
\hline 144 & 2015 & 2016 & & & & & 1 & 3 & & & & & 0 & 1 & & & & \\
\hline 147 & 2015 & 2016 & & & & & 0 & 0 & & & & & 0 & 0 & & & & \\
\hline 176 & 2013 & 2014 & 2015 & 2016 & & & 3 & 1 & 5 & 5 & & & 2 & 0 & 2 & 2 & & \\
\hline 179 & 2012 & 2013 & 2014 & 2015 & 2016 & & 6 & 7 & 10 & 7 & 13 & & 2 & 5 & 4 & 1 & 2 & \\
\hline 259 & 2014 & 2015 & 2016 & & & & 1 & 2 & 3 & & & & 0 & 1 & 1 & & & \\
\hline 265 & 2014 & 2015 & 2016 & & & & 0 & 0 & 3 & & & & 0 & 0 & 0 & & & \\
\hline 266 & 2015 & 2016 & & & & & 7 & 7 & & & & & 0 & 4 & & & & \\
\hline 273 & 2015 & 2016 & & & & & 1 & 8 & & & & & 1 & 6 & & & & \\
\hline 275 & 2016 & & & & & & 1 & & & & & & 1 & & & & & \\
\hline 277 & 2015 & 2016 & & & & & 1 & 11 & & & & & 1 & 4 & & & & \\
\hline 279 & & 2016 & & & & & & 9 & & & & & & 4 & & & & \\
\hline 283 & & 2015 & 2016 & & & & & 5 & 13 & & & & & 2 & 5 & & & \\
\hline
\end{tabular}




\section{APPENDIX C: INTERSECTION DATA FOR RISK ASSESSMENT MODELS}

Table C1: Turning-Movement Counts for Intersection A

\begin{tabular}{|c|c|c|c|c|c|c|c|c|c|c|c|c|}
\hline & \multicolumn{3}{|c|}{ Southbound } & \multicolumn{3}{|c|}{ Westbound } & \multicolumn{3}{|c|}{ Northbound } & \multicolumn{3}{|c|}{ Eastbound } \\
\hline & 6 & 6 & 1 & 8 & 8 & 3 & 2 & 2 & 5 & 4 & 4 & 7 \\
\hline Start & Right & Thru & Left & Right & Thru & Left & Right & Thru & Left & Right & Thru & Left \\
\hline $0: 00$ & 12 & 24 & 6 & 3 & 11 & 5 & 1 & 28 & 0 & 1 & 0 & 4 \\
\hline 1:00 & 7 & 10 & 4 & 4 & 9 & 7 & 4 & 20 & 2 & 3 & 0 & 2 \\
\hline $2: 00$ & 6 & 8 & 1 & 2 & 7 & 5 & 4 & 8 & 4 & 1 & 0 & 1 \\
\hline $3: 00$ & 1 & 7 & 1 & 1 & 5 & 2 & 1 & 8 & 2 & 0 & 0 & 2 \\
\hline $4: 00$ & 2 & 8 & 2 & 0 & 4 & 6 & 17 & 16 & 4 & 1 & 0 & 0 \\
\hline $5: 00$ & 6 & 15 & 5 & 0 & 43 & 35 & 88 & 45 & 6 & 0 & 0 & 2 \\
\hline $6: 00$ & 20 & 44 & 37 & 14 & 104 & 104 & 233 & 95 & 24 & 4 & 0 & 4 \\
\hline $7: 00$ & 104 & 101 & 23 & 24 & 249 & 128 & 143 & 205 & 155 & 13 & 5 & 12 \\
\hline $8: 00$ & 140 & 174 & 48 & 10 & 260 & 99 & 113 & 284 & 190 & 20 & 9 & 30 \\
\hline 9:00 & 118 & 212 & 35 & 12 & 151 & 75 & 46 & 262 & 108 & 25 & 9 & 41 \\
\hline 10:00 & 87 & 270 & 23 & 19 & 95 & 84 & 47 & 305 & 73 & 22 & 6 & 26 \\
\hline 11:00 & 107 & 273 & 33 & 56 & 139 & 99 & 55 & 327 & 70 & 40 & 7 & 64 \\
\hline $12: 00$ & 149 & 356 & 54 & 68 & 168 & 97 & 62 & 402 & 97 & 54 & 10 & 89 \\
\hline 13:00 & 121 & 319 & 41 & 41 & 132 & 91 & 88 & 395 & 108 & 39 & 7 & 53 \\
\hline $14: 00$ & 110 & 308 & 29 & 28 & 175 & 121 & 103 & 414 & 80 & 30 & 3 & 46 \\
\hline $15: 00$ & 130 & 339 & 25 & 41 & 216 & 156 & 36 & 384 & 75 & 28 & 5 & 51 \\
\hline $16: 00$ & 140 & 351 & 11 & 50 & 215 & 99 & 30 & 356 & 81 & 46 & 3 & 76 \\
\hline $17: 00$ & 180 & 384 & 6 & 68 & 300 & 126 & 25 & 410 & 92 & 66 & 10 & 105 \\
\hline 18:00 & 106 & 258 & 20 & 37 & 115 & 57 & 54 & 317 & 48 & 35 & 4 & 51 \\
\hline 19:00 & 67 & 207 & 9 & 12 & 69 & 41 & 10 & 243 & 53 & 24 & 0 & 36 \\
\hline $20: 00$ & 57 & 150 & 6 & 11 & 23 & 30 & 5 & 223 & 37 & 9 & 0 & 27 \\
\hline 21:00 & 62 & 134 & 12 & 9 & 20 & 23 & 24 & 130 & 20 & 5 & 1 & 21 \\
\hline 22:00 & 24 & 48 & 12 & 20 & 72 & 54 & 59 & 84 & 12 & 2 & 0 & 9 \\
\hline 23:00 & 28 & 34 & 3 & 9 & 54 & 29 & 3 & 42 & 5 & 5 & 0 & 15 \\
\hline
\end{tabular}

Table C2: Turning-Movement Counts for Intersection B

\begin{tabular}{|c|c|c|c|c|c|c|c|c|c|c|c|c|}
\hline & \multicolumn{3}{|c|}{ Southbound } & \multicolumn{3}{|c|}{ Westbound } & \multicolumn{3}{|c|}{ Northbound } & \multicolumn{3}{|c|}{ Eastbound } \\
\hline & 6 & 6 & 1 & 4 & 4 & 7 & 2 & 2 & 5 & 8 & 8 & 3 \\
\hline Start & Right & Thru & Left & Right & Thru & Left & Right & Thru & Left & Right & Thru & Left \\
\hline 0:00 & 0 & 74 & 0 & 0 & 0 & 0 & 0 & 68 & 1 & 1 & 0 & 0 \\
\hline 1:00 & 1 & 40 & 2 & 1 & 0 & 2 & 0 & 34 & 2 & 1 & 1 & 1 \\
\hline 2:00 & 0 & 27 & 1 & 0 & 0 & 0 & 0 & 18 & 0 & 0 & 0 & 0 \\
\hline $3: 00$ & 0 & 18 & 0 & 0 & 0 & 0 & 0 & 26 & 2 & 1 & 0 & 0 \\
\hline 4:00 & 0 & 15 & 1 & 1 & 0 & 0 & 0 & 30 & 0 & 0 & 0 & 0 \\
\hline 5:00 & 0 & 69 & 5 & 6 & 0 & 1 & 2 & 94 & 2 & 2 & 1 & 0 \\
\hline $6: 00$ & 1 & 205 & 26 & 13 & 0 & 4 & 4 & 275 & 2 & 2 & 0 & 2 \\
\hline 7:00 & 0 & 480 & 51 & 31 & 0 & 4 & 43 & 560 & 12 & 4 & 0 & 1 \\
\hline $8: 00$ & 3 & 594 & 62 & 30 & 0 & 25 & 62 & 693 & 22 & 18 & 4 & 4 \\
\hline 9:00 & 0 & 558 & 28 & 35 & 0 & 24 & 39 & 563 & 16 & 13 & 0 & 4 \\
\hline 10:00 & 1 & 574 & 31 & 42 & 1 & 27 & 37 & 561 & 5 & 11 & 2 & 3 \\
\hline 11:00 & 1 & 652 & 50 & 63 & 0 & 51 & 35 & 686 & 8 & 6 & 0 & 4 \\
\hline $12: 00$ & 1 & 819 & 73 & 75 & 0 & 41 & 34 & 843 & 9 & 18 & 2 & 5 \\
\hline $13: 00$ & 3 & 801 & 46 & 44 & 1 & 28 & 47 & 730 & 16 & 13 & 2 & 1 \\
\hline $14: 00$ & 2 & 747 & 47 & 39 & 0 & 35 & 29 & 730 & 13 & 12 & 0 & 5 \\
\hline $15: 00$ & 0 & 817 & 35 & 47 & 0 & 45 & 27 & 769 & 17 & 17 & 0 & 8 \\
\hline $16: 00$ & 2 & 879 & 26 & 60 & 1 & 56 & 25 & 801 & 18 & 15 & 1 & 8 \\
\hline $17: 00$ & 0 & 1129 & 8 & 65 & 2 & 80 & 8 & 980 & 25 & 37 & 2 & 6 \\
\hline 18:00 & 2 & 706 & 8 & 27 & 0 & 11 & 1 & 772 & 10 & 2 & 0 & 8 \\
\hline 19:00 & 7 & 539 & 5 & 4 & 0 & 7 & 0 & 542 & 4 & 4 & 0 & 4 \\
\hline 20:00 & 5 & 405 & 3 & 1 & 0 & 3 & 2 & 439 & 6 & 5 & 0 & 0 \\
\hline 21:00 & 3 & 376 & 6 & 5 & 0 & 3 & 2 & 304 & 4 & 3 & 0 & 2 \\
\hline $22: 00$ & 3 & 197 & 6 & 3 & 0 & 2 & 0 & 178 & 5 & 3 & 0 & 0 \\
\hline $23: 00$ & 0 & 103 & 0 & 0 & 0 & 1 & 0 & 114 & 1 & 3 & 0 & 1 \\
\hline
\end{tabular}


Table C3: Turning-Movement Counts for Intersection C

\begin{tabular}{|c|c|c|c|c|c|c|c|c|c|c|c|c|}
\hline & \multicolumn{3}{|c|}{ Southbound } & \multicolumn{3}{|c|}{ Westbound } & \multicolumn{3}{|c|}{ Northbound } & \multicolumn{3}{|c|}{ Eastbound } \\
\hline & 2 & 2 & 5 & 8 & 8 & 8 & 6 & 6 & 1 & 4 & 4 & 4 \\
\hline Start & Right & Thru & Left & Right & Thru & Left & Right & Thru & Left & Right & Thru & Left \\
\hline $0: 00$ & 5 & 139 & 3 & 2 & 0 & 2 & 0 & 83 & 0 & 1 & 0 & 1 \\
\hline $1: 00$ & 2 & 83 & 0 & 2 & 1 & 1 & 3 & 61 & 0 & 0 & 0 & 1 \\
\hline 2:00 & 1 & 77 & 0 & 0 & 0 & 0 & 2 & 68 & 0 & 0 & 0 & 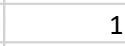 \\
\hline $3: 00$ & 2 & 67 & 1 & 0 & 0 & 1 & 0 & 82 & 0 & 0 & 0 & 1 \\
\hline 4:00 & 3 & 113 & 11 & 4 & 0 & 0 & 4 & 171 & 0 & 0 & 0 & 5 \\
\hline $5: 00$ & 3 & 184 & 13 & 2 & 0 & 1 & 3 & 468 & 0 & 1 & 1 & 12 \\
\hline 6:00 & 4 & 380 & 39 & 15 & 1 & 3 & 14 & 1093 & 2 & 1 & 1 & 36 \\
\hline $7: 00$ & 16 & 694 & 104 & 45 & 4 & 24 & 33 & 1642 & 7 & 6 & 19 & 88 \\
\hline $8: 00$ & 37 & 676 & 108 & 48 & 7 & 17 & 45 & 1415 & 16 & 5 & 19 & 82 \\
\hline 9:00 & 51 & 775 & 119 & 62 & 8 & 26 & 48 & 1021 & 6 & 5 & 16 & 76 \\
\hline $10: 00$ & 58 & 762 & 95 & 51 & 5 & 32 & 68 & 991 & 8 & 6 & 19 & 62 \\
\hline 11:00 & 52 & 882 & 163 & 75 & 14 & 54 & 75 & 963 & 13 & 5 & 23 & 63 \\
\hline $12: 00$ & 84 & 999 & 214 & 94 & 14 & 77 & 74 & 919 & 11 & 12 & 29 & 69 \\
\hline $13: 00$ & 64 & 1047 & 212 & 88 & 28 & 86 & 76 & 984 & 16 & 13 & 38 & 67 \\
\hline $14: 00$ & 85 & 1142 & 192 & 89 & 13 & 100 & 76 & 953 & 8 & 14 & 22 & 59 \\
\hline $15: 00$ & 80 & 1339 & 183 & 97 & 20 & 78 & 70 & 896 & 10 & 9 & 23 & 68 \\
\hline $16: 00$ & 82 & 1597 & 197 & 86 & 30 & 112 & 74 & 926 & 16 & 15 & 37 & 50 \\
\hline $17: 00$ & 99 & 1664 & 209 & 81 & 38 & 159 & 84 & 947 & 12 & 36 & 38 & 83 \\
\hline $18: 00$ & 84 & 1055 & 162 & 59 & 16 & 117 & 56 & 793 & 21 & 21 & 21 & 50 \\
\hline 19:00 & 69 & 875 & 132 & 37 & 11 & 72 & 47 & 617 & 20 & 12 & 12 & 50 \\
\hline $20: 00$ & 49 & 661 & 87 & 33 & 13 & 60 & 33 & 410 & 14 & 7 & 8 & 22 \\
\hline 21:00 & 20 & 517 & 31 & 18 & 4 & 38 & 11 & 324 & 7 & 2 & 4 & 13 \\
\hline $22: 00$ & 10 & 305 & 27 & 11 & 4 & 17 & 6 & 267 & 1 & 1 & 0 & 5 \\
\hline $23: 00$ & 10 & 234 & 13 & 14 & 1 & 7 & 4 & 158 & 2 & 0 & 1 & 4 \\
\hline
\end{tabular}

Table C4: Turning-Movement Counts for Intersection D

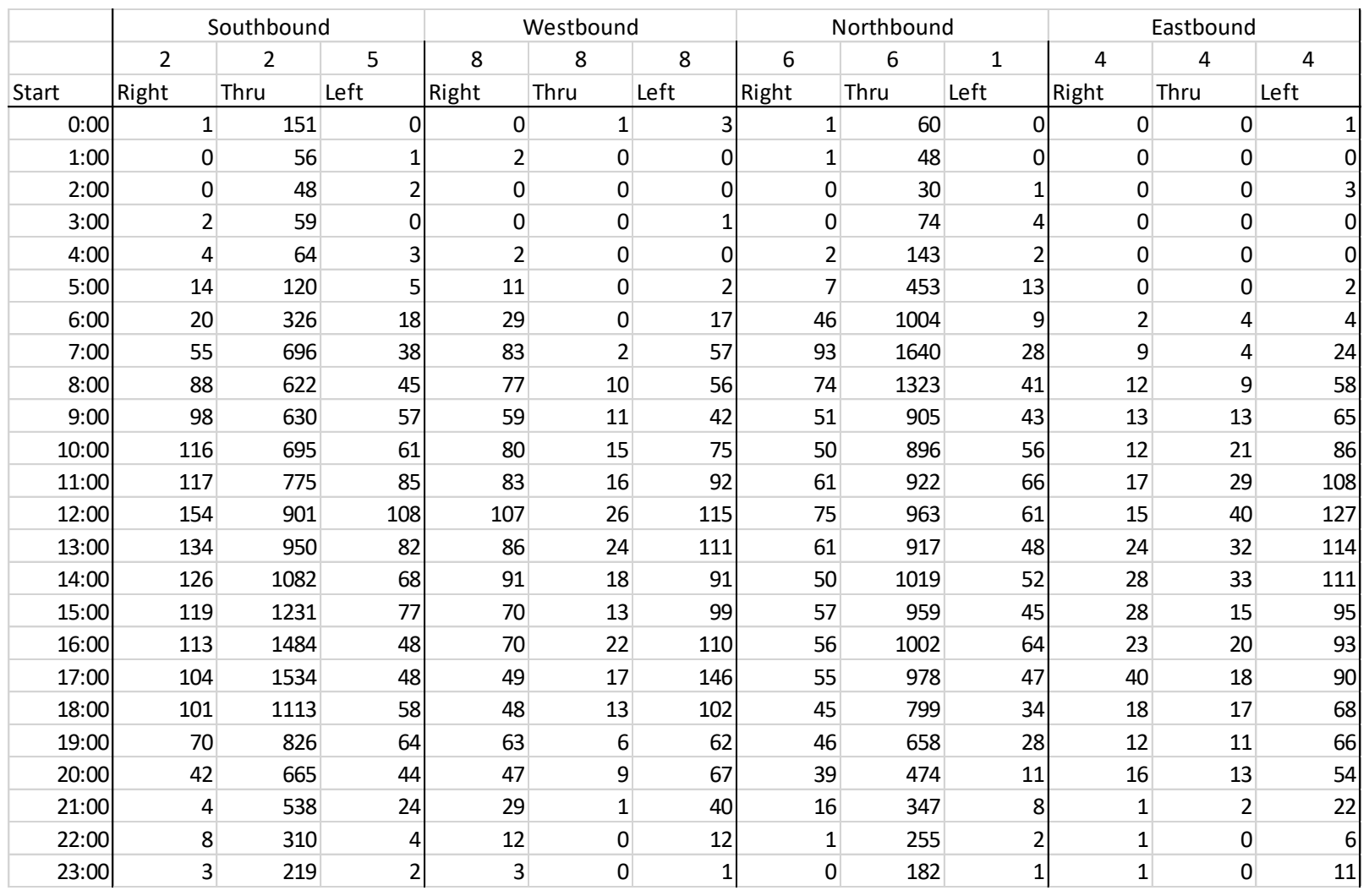


Table C5: Turning-Movement Counts for Intersection E

\begin{tabular}{|c|c|c|c|c|c|c|c|c|c|c|c|c|}
\hline & \multicolumn{3}{|c|}{ Southbound } & \multicolumn{3}{|c|}{ Westbound } & \multicolumn{3}{|c|}{ Northbound } & \multicolumn{3}{|c|}{ Eastbound } \\
\hline & 2 & 2 & 5 & 8 & 8 & 3 & 6 & 6 & 1 & 4 & 4 & 7 \\
\hline Start & Right & Thru & Left & Right & Thru & Left & Right & Thru & Left & Right & Thru & Left \\
\hline $0: 00$ & 1 & 15 & 5 & 0 & 6 & 19 & 4 & 21 & 5 & 14 & 5 & 1 \\
\hline $1: 00$ & 2 & 6 & 4 & 4 & 4 & 6 & 6 & 7 & 3 & 3 & 2 & 0 \\
\hline $2: 00$ & 1 & 5 & 2 & 1 & 0 & 8 & 3 & 6 & 2 & 3 & 2 & 1 \\
\hline $3: 00$ & 0 & 9 & 0 & 1 & 1 & 4 & 3 & 13 & 3 & 2 & 1 & 0 \\
\hline 4:00 & 0 & 7 & 2 & 1 & 5 & 2 & 20 & 45 & 7 & 2 & 4 & 0 \\
\hline $5: 00$ & 1 & 16 & 8 & 7 & 5 & 6 & 49 & 125 & 25 & 5 & 5 & 2 \\
\hline $6: 00$ & 0 & 80 & 11 & 22 & 21 & 22 & 182 & 353 & 133 & 59 & 10 & 6 \\
\hline 7:00 & 14 & 134 & 39 & 47 & 103 & 74 & 399 & 606 & 277 & 97 & 71 & 21 \\
\hline $8: 00$ & 6 & 192 & 52 & 51 & 76 & 84 & 328 & 470 & 270 & 108 & 74 & 11 \\
\hline $9: 00$ & 9 & 229 & 51 & 60 & 58 & 119 & 274 & 405 & 183 & 122 & 69 & 22 \\
\hline $10: 00$ & 17 & 247 & 60 & 49 & 57 & 170 & 251 & 393 & 166 & 141 & 74 & 25 \\
\hline $11: 00$ & 8 & 291 & 84 & 60 & 73 & 180 & 207 & 451 & 131 & 163 & 74 & 20 \\
\hline $12: 00$ & 16 & 381 & 90 & 76 & 79 & 218 & 239 & 454 & 141 & 151 & 98 & 36 \\
\hline $13: 00$ & 17 & 412 & 83 & 64 & 77 & 207 & 214 & 435 & 135 & 154 & 95 & 21 \\
\hline $14: 00$ & 21 & 373 & 77 & 55 & 68 & 233 & 200 & 381 & 140 & 174 & 92 & 34 \\
\hline $15: 00$ & 23 & 483 & 100 & 61 & 94 & 267 & 190 & 384 & 149 & 201 & 93 & 34 \\
\hline $16: 00$ & 25 & 519 & 91 & 44 & 91 & 300 & 193 & 385 & 165 & 289 & 143 & 42 \\
\hline $17: 00$ & 11 & 708 & 110 & 59 & 108 & 349 & 208 & 429 & 177 & 329 & 140 & 46 \\
\hline $18: 00$ & 15 & 509 & 70 & 46 & 66 & 330 & 201 & 356 & 111 & 187 & 89 & 24 \\
\hline $19: 00$ & 19 & 349 & 44 & 32 & 53 & 280 & 142 & 208 & 87 & 129 & 56 & 20 \\
\hline $20: 00$ & 14 & 289 & 34 & 27 & 62 & 199 & 74 & 153 & 62 & 88 & 29 & 11 \\
\hline 21:00 & 9 & 173 & 21 & 20 & 30 & 140 & 35 & 94 & 39 & 49 & 27 & 4 \\
\hline 22:00 & 3 & 89 & 16 & 7 & 14 & 79 & 22 & 51 & 18 & 33 & 12 & 4 \\
\hline 23:00 & 0 & 52 & 8 & 1 & 6 & 33 & 11 & 36 & 14 & 25 & 4 & 0 \\
\hline
\end{tabular}

Table C6: Turning-Movement Counts for Intersection F

\begin{tabular}{|c|c|c|c|c|c|c|}
\hline & \multicolumn{2}{|c|}{ Southbound } & \multicolumn{2}{|c|}{ Westbound } & \multicolumn{2}{|c|}{ Northbound } \\
\hline & 2 & 5 & 4 & 4 & 6 & 6 \\
\hline Start & Thru & Left & Right & Left & Right & Thru \\
\hline $0: 00$ & 141 & 4 & 3 & 0 & 0 & 79 \\
\hline $1: 00$ & 76 & 5 & 3 & 1 & 0 & 63 \\
\hline $2: 00$ & 76 & 4 & 1 & 1 & 0 & 66 \\
\hline $3: 00$ & 67 & 4 & 3 & 1 & 0 & 77 \\
\hline $4: 00$ & 113 & 2 & 9 & 0 & 0 & 162 \\
\hline $5: 00$ & 185 & 9 & 28 & 2 & 2 & 465 \\
\hline $6: 00$ & 350 & 27 & 81 & 11 & 5 & 1034 \\
\hline 7:00 & 681 & 37 & 169 & 35 & 5 & 1538 \\
\hline $8: 00$ & 659 & 51 & 162 & 31 & 5 & 1280 \\
\hline 9:00 & 733 & 74 & 110 & 28 & 11 & 995 \\
\hline $10: 00$ & 730 & 66 & 126 & 24 & 15 & 949 \\
\hline $11: 00$ & 833 & 94 & 119 & 32 & 12 & 974 \\
\hline $12: 00$ & 951 & 138 & 115 & 27 & 14 & 919 \\
\hline $13: 00$ & 1049 & 97 & 108 & 24 & 13 & 930 \\
\hline $14: 00$ & 1178 & 106 & 112 & 22 & 22 & 935 \\
\hline $15: 00$ & 1299 & 119 & 91 & 27 & 24 & 851 \\
\hline $16: 00$ & 1576 & 133 & 109 & 26 & 30 & 945 \\
\hline $17: 00$ & 1789 & 174 & 108 & 34 & 27 & 911 \\
\hline $18: 00$ & 1039 & 175 & 123 & 35 & 23 & 756 \\
\hline $19: 00$ & 827 & 121 & 75 & 23 & 15 & 586 \\
\hline 20:00 & 626 & 101 & 59 & 17 & 11 & 373 \\
\hline 21:00 & 487 & 65 & 28 & 10 & 5 & 283 \\
\hline 22:00 & 291 & 40 & 21 & 10 & 6 & 245 \\
\hline 23:00 & 231 & 13 & 11 & 3 & 1 & 152 \\
\hline
\end{tabular}

